\title{
A TAXONOMIC REVISION OF HYMENOPHYLLACEAE
}

\author{
ATSUSHI EBIHARA ${ }^{1,2}$, JEAN-YVES DUBUISSON ${ }^{3}$, KUNIO IWATSUKI ${ }^{4}$, \\ SABINE HENNEQUIN ${ }^{3} \&$ MOTOMI ITO $^{1}$
}

\begin{abstract}
SUMMARY
A new classification of Hymenophyllaceae, consisting of nine genera (Hymenophyllum, Didymoglossum, Crepidomanes, Polyphlebium, Vandenboschia, Abrodictyum, Trichomanes, Cephalomanes and Callistopteris) is proposed. Every genus, subgenus and section chiefly corresponds to the monophyletic group elucidated in molecular phylogenetic analyses based on chloroplast sequences. Brief descriptions and keys to the higher taxa are given, and their representative members are enumerated, including some new combinations.
\end{abstract}

Key words: filmy ferns, Hymenophyllaceae, Hymenophyllum, Trichomanes.

\section{INTRODUCTION}

The Hymenophyllaceae, or 'filmy ferns', is the largest basal family of leptosporangiate ferns and comprises around 600 species (Iwatsuki, 1990). Members are easily distinguished by their usually single-cell-thick laminae, and the monophyly of the family has not been questioned. The intrafamilial classification of the family, on the other hand, is highly controversial - several fundamentally different classifications are used by individual researchers and/or areas. Traditionally, only two genera-Hymenophyllum with bivalved involucres and Trichomanes with tubular involucres - have been recognized in this family. This scheme was expanded by Morton (1968) who hierarchically placed many subgenera, sections and subsections under two larger genera Hymenophyllum and Trichomanes and recognized four monotypic genera, Cardiomanes, Hymenoglossum, Rosenstockia and Serpyllopsis, and all of which have specialized fronds. Since his system carefully covered New World taxa, most of the recent floristic works of Central and South America adopted his scheme (Stolze, 1976; De la Sota, 1977; Kramer, 1978; Smith, 1981; Tryon \& Tryon, 1982; Proctor, 1985, 1989; Mickel \& Beitel, 1988; Lellinger, 1989; Tryon \& Stolze, 1989; Marticorena \& Rodriguez, 1995; Pacheco, 1995; Baksh-Comeau, 2000; Sánchez, 2000). Conversely, Copeland (1938b, 1947) split the

1) Department of System Sciences, Graduate School of Arts and Sciences, The University of Tokyo, 3-8-1 Komaba, Meguro-ku, Tokyo 153-8902, Japan. Present address: Department of Botany, The National Science Museum, 4-1-1 Amakubo, Tsukuba 305-0005, Japan; ebihara@kahaku.go.jp.

2) Research fellow of the Japan Society for the Promotion of Science.

3) Équipe 'Paléobiodiversité, Systématique et Évolution des Embryophytes' UMR 5143, Laboratoire de Paléobotanique et Paléoécologie, IFR 101 CNRS 'Institut d'Ecologie Fondamentale et Appliquée', Université Pierre et Marie Curie, 12 rue Cuvier, F-75005 Paris, France.

4) The Museum of Nature and Human Activities, Hyogo, Yayoigaoka 6-chome, Sanda 669-1546, Japan. 
family into 34 genera, basing his work mainly on in situ and herbarium observation of Asian material. His scheme has been adopted in floristic works of Asia (Copeland, 1958; Ching, 1959; De Vol, 1975; Nakaike, 1975; Tagawa \& Iwatsuki, 1979; Parris, 1992), but has not yet been applied to New World taxa. Another system consisting of 47 genera was proposed by Pichi Sermolli (1977b), but it has been adopted only in a limited number of publications on African plants (e.g., Pichi Sermolli, 1983). Finally, Iwatsuki integrated a series of his studies of morphological characters (Iwatsuki, 1975, 1977a, b, 1978, 1981, 1982) into a new hierarchical system consisting of eight genera (Iwatsuki, 1984, 1985). Although his system has been adopted in a few recently published floristic works (Iwatsuki, 1995; Du Puy \& Orchard, 1993; Green, 1994; Bostock $\&$ Spokes, 1998), generic combinations for taxa in regions other than Asia and Australia are still wanting. These taxonomic inconsistencies were compounded with the typification problem of the Linnaean genus Trichomanes (see Holttum, 1976 and Pichi Sermolli, 1981) - Trichomanes crispum was considered the type in the systems devised by Copeland (1933, 1938b), Pichi Sermolli (1977b) and Iwatsuki (1984), while T. scandens was considered the type by Morton (1968) and Nakaike (1975).

Recent molecular phylogenetic studies (Dubuisson, 1997; Pryer et al., 2001; Dubuisson et al., 2003a; Hennequin et al., 2003; Ebihara et al., 2004; Ebihara et al., submitted) have provided new insights into the systematic relationships within the Hymenophyllaceae. The presence of two large monophyletic groups (Pryer et al., 2001) can be interpreted to support the traditional bigeneric system; however some traditional Trichomanes taxa (Cardiomanes, Microtrichomanes, Pleuromanes) belong in fact to the Hymenophyllum lineage (Pryer et al., 2001; Hennequin et al., 2003; Ebihara et al., 2004; Ebihara et al., submitted). Moreover, the trichomanoid and hymenophylloid lineages, both consisting of nearly the same number of taxa, may possess different historical backgrounds - the Hymenophyllum lineage has likely diversified more recently than the Trichomanes lineage (Ebihara et al., 2004; Schuettpelz \& Pryer, in press). This may explain why the Trichomanes lineage displays a greater variety of morphological features and ecological habits than the Hymenophyllum lineage. Earlier studies on Trichomanes (Dubuisson, 1997; Dubuisson et al., 2003a), as well as our latest study (Ebihara et al., submitted), also based on $r b c L$ sequences and representing nearly all taxonomic lineages, did not support any of the above classifications. These inconsistencies are probably caused by strong homoplasy of taxonomic characters due to adaptive evolution (Dubuisson et al. 2003b; Ebihara et al., submitted). We therefore believe that a fundamental taxonomic revision of the Hymenophyllaceae is in order.

Even today, the principles of generic delimitation maintained by Copeland (1938b) seem quite reasonable: naturalness and convenience, and the latter is "subject always to the preceding principle (naturalness)". It is often argued that a classification completely based on the results of molecular phylogeny is difficult to put into practice, but recognition of unnatural (i.e., polyphyletic/paraphyletic) groups is a disadvantage for biological studies in general. Hence we prefer to reorganize the species into natural (i.e., monophyletic) and also morphologically recognizable groups. We propose to place all species of the hymenophylloid lineage under a single genus Hymenophyllum and to subdivide the trichomanoid lineage into eight genera corresponding to eight robust clades in our global analysis (Ebihara et al., submitted). We also treat many of the subclades retrieved (Ebihara et al., submitted) as subgenera if relationships within the 


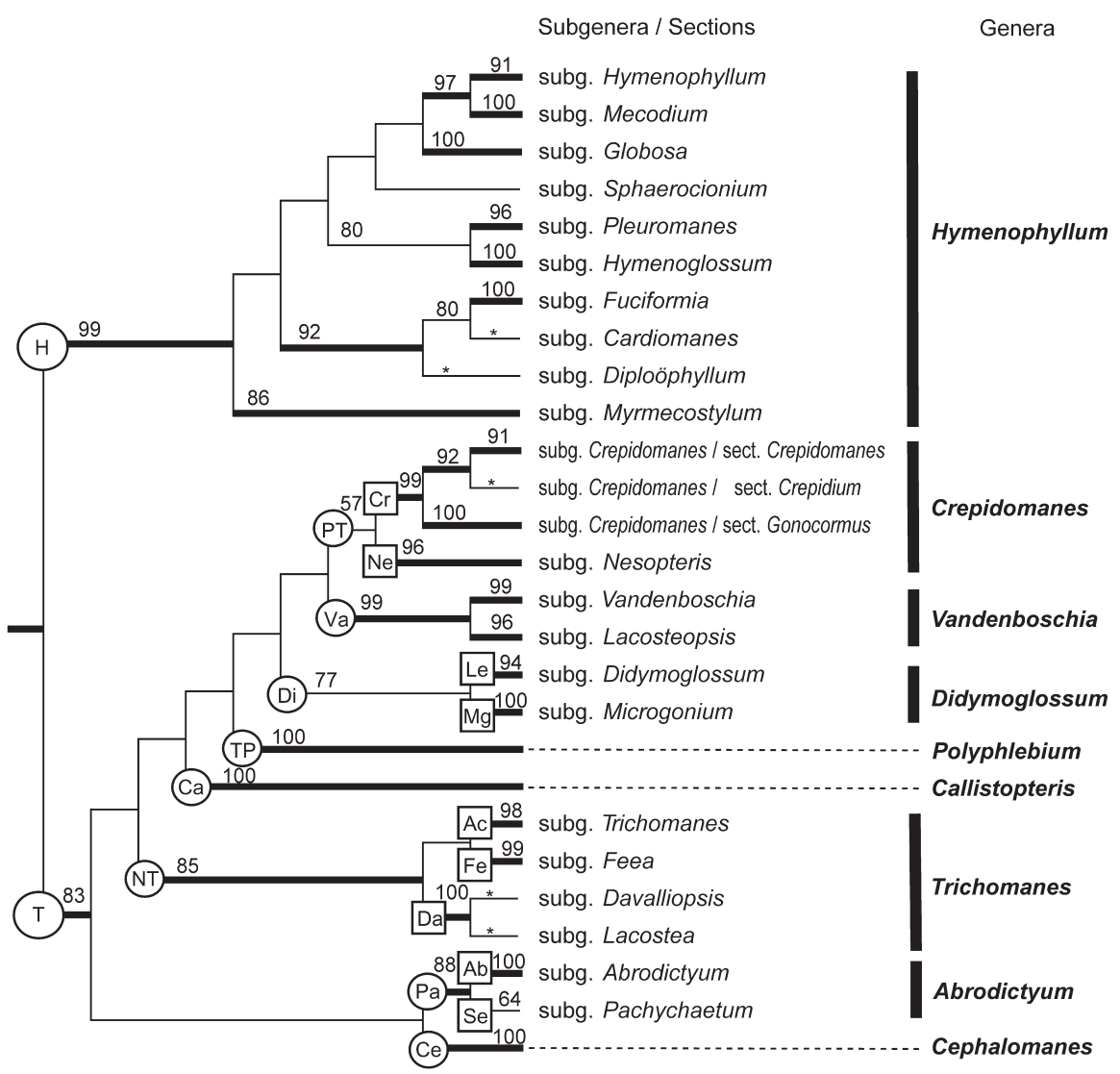

Fig. 1. An explanatory figure of the correspondence between the present system and the results of molecular phylogeny of Hymenophyllaceae. The phylogram is primarily based on the MP tree using chloroplast $r b c L$ gene (Ebihara et al., submitted); the topology within the ' $H$ ' clade follows that of the MP tree using $r b c L, r b c L-a c c D$ and $r p s 4$-trnS regions (Hennequin et al., in press). Bootstrap values (>50\%) supporting the clades are shown on their branches (bootstrap supports $>80 \%$ are shown by bold lines), but the values are replaced by asterisks in the taxa comprising a single sample. All the groups adopted as taxa in the present system are supported by high posterior probabilities $(>0.99)$ of Bayesian inference (Ebihara et al., submitted; Hennequin et al., in press). The clade names indicated in the tree correspond to those used in Ebihara et al. (submitted). - Ab: Abrodictyum; Ac: Achomanes; Ca: Callistopteris; Ce: Cephalomanes; Cr: Crepidomanes; Da: Davalliopsis; Di: Didymoglossum; Fe: Feea; H: Hymenophyllum s.l.; Le: Lecanium; Mg: Microgonium; Ne: Nesopteris; NT: Neotropical; Pa: Pachychaetum; PT: Paleotropical; Se: Selenodesmium; T: Trichomanes s.1.; TP: Trichomanes pixidiferum; Va: Vandenboschia.

clade are well resolved in our analysis (Fig. 1); in the absence of support, infrageneric classification within some genera is deferred. Although Ebihara et al. (submitted) illustrated that anatomical and cytological characters are more taxonomically informative than macroscopic characters, we selected macroscopic characters for the keys in this paper even though this makes the keys more complex. 
To aid in characterization of taxa recognized by us, representative species of each genus, subgenus or section are enumerated here. Please note that the list includes the minimum number of taxa commonly accepted in recent floristic works, and therefore does not necessarily represent all the members.
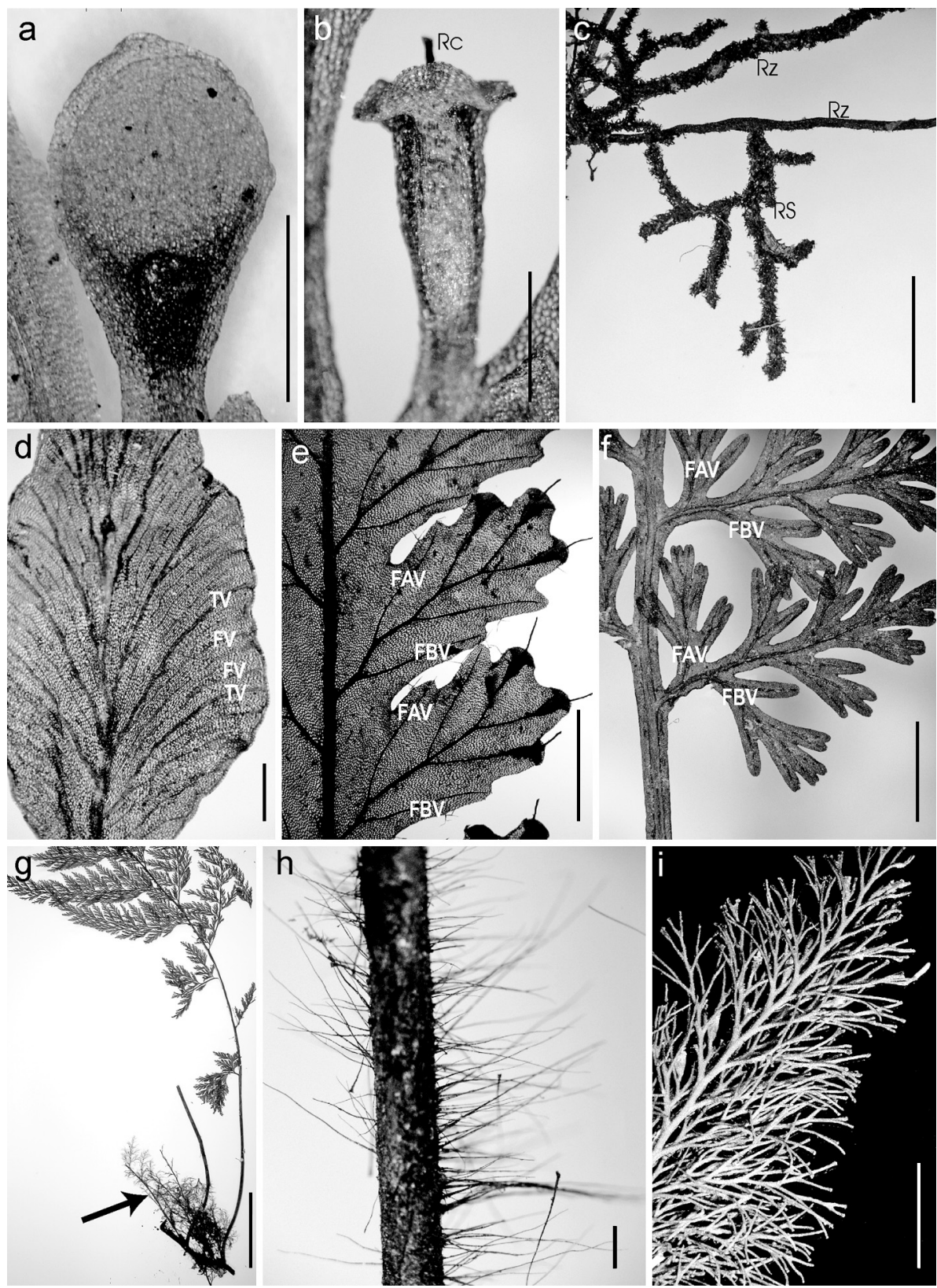


\section{TAXONOMIC TREATMENT}

\section{KEY TO THE GENERA}

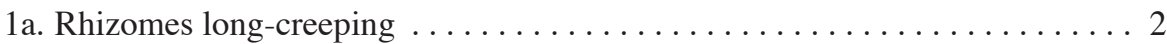

b. Rhizomes short-creeping or erect $\ldots \ldots \ldots \ldots \ldots \ldots \ldots \ldots \ldots \ldots$

2a. Rhizomes nearly glabrous or sparsely covered with light-coloured hairs. Involucres usually bivalvate (Fig. 2a) . . . . . . . . . . . . 1. Hymenophyllum

b. Rhizomes covered with reddish to dark-coloured hairs. Involucres usually not

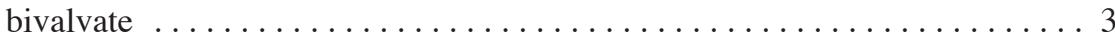

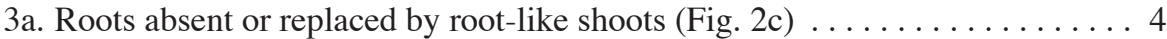

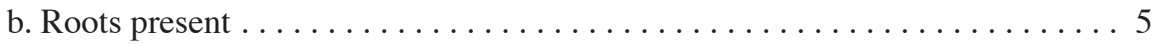

4a. Continuous false veinlets parallel to true veins (Fig. 2d) present. Blade venation

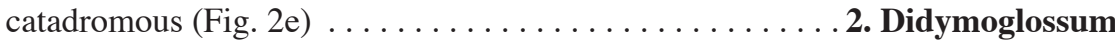

b. Continuous false veinlets parallel to true veins absent. Blade venation anadromous (Fig. 2f). . . . . . . . . . 3. Crepidomanes (subg. Crepidomanes)

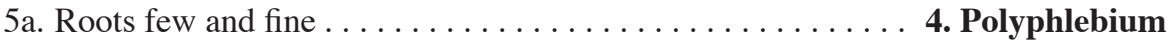

b. Roots numerous and robust $\ldots \ldots \ldots \ldots \ldots \ldots \ldots \ldots \ldots \ldots \ldots \ldots \ldots \ldots \ldots \ldots \ldots$

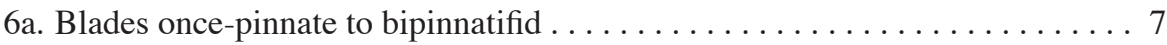

b. Blades bipinnate to highly divided $\ldots \ldots \ldots \ldots \ldots \ldots \ldots \ldots$

7a. Pinnae asymmetric $\ldots \ldots \ldots \ldots \ldots \ldots \ldots \ldots$ 5. Vandenboschia

b. Pinnae symmetric . . . . . . . . . . 7. Trichomanes (subg. Lacostea)

8a. Marginal laminar trichomes present . . . 7. Trichomanes (subg. Trichomanes)

b. Marginal laminar trichomes absent . . . . . . . . . . . . . . . . . . 9

9a. Abortive fronds (Fig. 2g) present. . . . . 3. Crepidomanes (subg. Nesopteris)

b. Abortive fronds absent . . . . . . . . . . . . . . . . . . . . 10

10a. Minute clavate hairs present on stipes and rachises .... 5. Vandenboschia

b. Minute clavate hairs on stipes and rachises absent .......6. Abrodictyum

11a. Involucres bivalvate (Fig. 2a) . . . . . 1. Hymenophyllum (subg. Fuciformia)

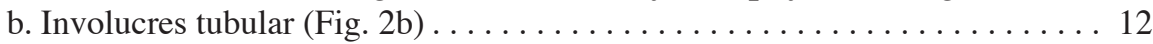

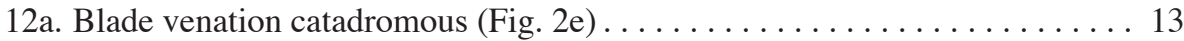

b. Blade venation anadromous (Fig. 2f) . . . . . . . . . . . . . . . . 14

13a. Lamina tough, more than one cell thick ...... 7. Trichomanes (subg. Feea)

b. Lamina membranous, one cell thick ... . 7. Trichomanes (subg. Trichomanes)

Fig. 2. Examples of morphological characters used in the keys. a. Bivalvate involucres (Hymenophyllum deplanchei; Ebihara 001228-05, TI); b. tubular involucres with an exserted receptacle (Rc) (Crepidomanes humile; Iwatsuki 99H06, TNS); c. root-like shoots (RS) grown out from rhizomes (Rz) (Crepidomanes bipunctatum; Ebihara 000225-020, TNS); d. continuous false veinlets (FV) parallel to true veins (TV) (Didymoglossum bipunctatum; S. Matsumoto 01-820, TNS); e. catadromous venation - FAV: the first acroscopic vein; FBV: the first basiscopic vein in each pinna (Trichomanes polypodioides; T.A. Ohsawa 178-15, TNS); f. anadromous venation (Vandenboschia birmanica; A. Narita $1070 b$, TNS); g. abortive fronds (pointed by an arrow) (Crepidomanes aphlebioides; M. Kato et al. C-13883, TI); h. long bristle-like light reddish hairs on a stipe (Callistopteris apiifolia; Ebihara 040922-03, TNS); i. reduced lamina cells (Abrodictyum pluma; R. Jaman s.n., TNS). - Scale bars: $\mathrm{a}, \mathrm{b}, \mathrm{d}, \mathrm{h}=1 \mathrm{~mm} ; \mathrm{c}, \mathrm{e}, \mathrm{f}, \mathrm{i}=5 \mathrm{~mm} ; \mathrm{g}=5 \mathrm{~cm}$. 
14a. Blades once-pinnate to bipinnatifid . . . . . . . . . Cephalomanes

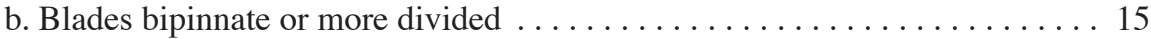

15a. Long bristle-like light reddish hairs (Fig. 2h) present on stipes and rachises . 16

b. Long bristle-like light reddish hairs absent on stipes and rachises . . . . . . 17

16a. Laminar cells reduced (Fig. 2i). Less than 3 rows of cells between midribs and

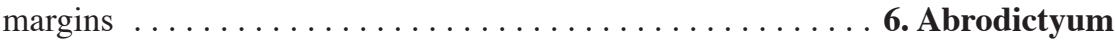

b. Laminar cells not reduced. More than 3 rows of cells between midribs and margins $\ldots \ldots \ldots \ldots \ldots \ldots \ldots \ldots \ldots \ldots \ldots \ldots \ldots \ldots \ldots \ldots \ldots \ldots \ldots$. Callistopteris

17a. Laminae more than one cell thick . . . . 7. Trichomanes (subg. Davalliopsis)

b. Laminae one cell thick . . . . . . . . . . . . . . . . . . . . . 18

18a. Laminar cell-walls thin and straight ... 2. Crepidomanes (subg. Nesopteris)

b. Laminar cell-walls slightly or quite thick, wavy or pitted... . 6. Abrodictyum

\section{HYMENOPHYLLUM}

Hymenophyllum Sm. (1793) 418. - Lectotype: Hymenophyllum tunbrigense (L.) Sm. (selected by Presl (1843) 31).

Rhizomes long-creeping, frequently branching, usually filiform or wiry, up to $2 \mathrm{~mm}$ diam., nearly glabrous or sparsely covered with multicellular hairs (exceptionally densely covered with hairs in subg. Fuciformia), protostele subcollateral, dorsiventral, or 'reduced' (with more or less central xylem-like parenchyma), cortices heterogeneous, with few and fine roots. Stipes various in length, at a distance from the adjacent ones. Blades usually pinnate to quadripinnate or occasionally simple, 165 by $17 \mathrm{~cm}$, venation anadromous, laminae usually one cell thick, but sometimes two or more cells thick. Sori paratactic, lips usually bivalvate, receptacles usually included in involucres.

Distribution - Throughout the tropics to temperate regions; c. 250 species.

Habitat - Usually low- to middle-epiphytes on tree trunks, sometimes canopy sunepiphytes, occasionally epilithic or terrestrial.

Chromosome base number - Various, $\mathrm{x}=11$ to 36 .

Note - Hymenophyllum in our sense stands as the largest genus of Hymenophyllaceae, and almost corresponds to Hymenophyllum in the traditional and broad sense but also includes some taxa formerly regarded as Trichomanes s.l. Iwatsuki (1984) segregated Sphaerocionium, which is characterized by distinctive stellate hairs, from the remaining Hymenophyllum (s.1.) taxa. Recent studies (Hennequin et al., 2003, in press) suggest that Sphaerocionium is nested within Mecodium, another easily recognizable group of Hymenophyllum s.1., as currently defined. Under our strategy of classification - making each taxon a natural group - Sphaerocionium is to be treated as a genus, species belonging to Mecodium should be split into more than two genera. To avoid such inconvenience, all species of Hymenophyllum s.l. are placed in our Hymenophyllum. Because the systematic relationships within this genus are not fully clarified, the current revision treats only subgenera, with each subgenus monophyletic based on our latest study using molecular data (Hennequin et al., in press). 


\section{KEY TO THE SUBGENERA}

1a. Rhizomes short-creeping or erect $\ldots \ldots \ldots \ldots \ldots \ldots$ 8. Subg. Fuciformia

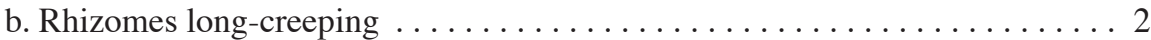

2a. Stellate hairs present on blades .......... 2. Subg. Sphaerocionium

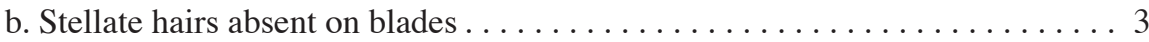

3a. Blades dichotomously divided ........... 2. Subg. Sphaerocionium

b. Blades not dichotomously divided $\ldots \ldots \ldots \ldots \ldots \ldots \ldots \ldots$

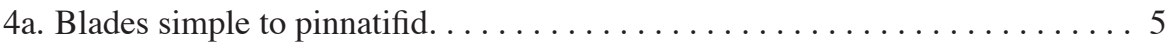

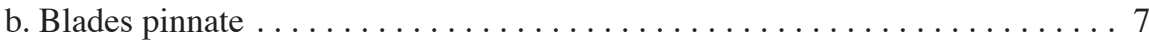

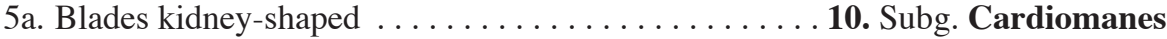

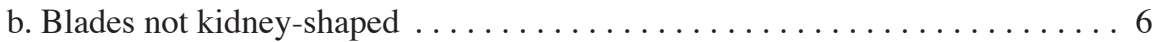

6a. Veins of blades simple . . . . . . . . . . . Subg. Hymenophyllum

b. Veins of blades pinnately divided ........... 7. Subg. Hymenoglossum

7a. Blades glaucous or covered with whitish multicellular hairs $\ldots \ldots \ldots \ldots \ldots$

5. Subg. Pleuromanes

b. Blades neither glaucous nor covered with whitish multicellular hairs . . . . 8

8a. Laminae more than two cells thick throughout ..... 9. Subg. Diploöphyllum

b. Laminae one cell thick, or only partly multi-layered. . . . . . . . . . . . . 9

9a. Stipes with multicellular hairs present $\ldots \ldots \ldots \ldots \ldots \ldots \ldots \ldots \ldots \ldots$

b. Stipes glabrous or with only caducous hairs $\ldots \ldots \ldots \ldots \ldots \ldots \ldots \ldots$

10a. Sori on both acroscopic and basiscopic sides of pinnae

6. Subg. Myrmecostylum

b. Sori confined to acroscopic sides of pinnae .... . 1. Subg. Hymenophyllum

11a. Rhizomes thick, more than $0.4 \mathrm{~mm}$ diam., nearly glabrous or with scattered pale hairs ...................... Subg. Globosa

b. Rhizomes filiform, less than $0.4 \mathrm{~mm}$ diam., with scattered short brown hairs 12

12a. Margins of segments toothed .............. 1. Subg. Hymenophyllum

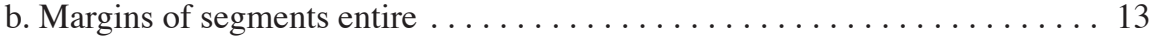

13a. Lamina cell walls thin and straight, sori lacking a thickened base . . . . . . . .

3. Subg. Mecodium

b. Lamina cell walls more or less thick, sori each with a thickened base, on short acroscopic segments near the rachis

1. Subg. Hymenophyllum

\section{Subgenus Hymenophyllum}

Didymoglossum Desv. subg. Chilodium C. Presl (1843) 23. - Hymenophyllum Sm. subg. Chilodium (C. Presl) Croxall (1975) 515. - Hymenophyllum Sm. sect. Chilodium (C. Presl) C. Chr. (1906) XVI. - Lectotype: Didymoglossum neesii (Blume) C. Presl (= Hymenophyllum denticulatum Sw.) (selected by Christensen (1906) XVI, as H. denticulatum Sw.).

Meringium C. Presl (1843) 24, t. VIII, f. B. - Hymenophyllum Sm. subg. Meringium (C. Presl) Copel. (1937) 14. - Hymenophyllum Sm. sect. Meringium (C. Presl) Diem \& J.S. Licht. (1959) 666.

- Type: Meringium meyenianum C. Presl (= Hymenophyllum serrulatum (C. Presl) C. Chr.).

Leptocionium C. Presl (1843) 26, t. XI, f. D. - Hymenophyllum Sm. sect. Leptocionium (C. Presl)

Prantl (1875) 54. - Hymenophyllum Sm. subg. Leptocionium (C. Presl) H. Christ (1897) 20.

- Hymenophyllum Sm. subsect. Leptocionium (C. Presl) C.V. Morton (1968) 170. - Type: Leptocionium dicranotrichum C. Presl (= Hymenophyllum dicranotrichum (C. Presl) Sadeb.). 
Hymenophyllum Sm. subg. Sphaerodium C. Presl (1843) 31. - Hymenophyllum Sm. sect. Sphaerodium (C. Presl) C. Chr. (1906) XVI. - Lectotype: Hymenophyllum wilsonii Hook. (selected by Copeland (1938b) 14).

Hymenophyllum Sm. subsect. Dimidiata C. Presl (1843) 32. - Lectotype: Hymenophyllum unilaterale Bory ex Willd. (= Hymenophyllum peltatum (Poir.) Desv.) (selected by Morton (1968) 164).

Hymenophyllum Sm. subg. Cycloglossum C. Presl (1843) 32. - Hymenophyllum Sm. sect. Cycloglossum (C. Presl) C. Chr. (1906) XV. - Lectotype: Hymenophyllum caespitosum Gaudich. (selected by Copeland (1938b) 14).

Hymenophyllum Sm. subg. Craspedophyllum C. Presl (1843) 33. - Hymenophyllum Sm. sect. Craspedophyllum (C. Presl) C. Chr. (1906) XV. - Craspedophyllum (C. Presl) Copel. (1938b) 27. - Type: Hymenophyllum marginatum Hook. \& Grev.

Serpyllopsis Bosch (1861a) 318. - Trichomanes L. subg. Serpyllopsis (Bosch) H. Christ (1897) 23. - Type: Serpyllopsis caespitosa (Gaudich.) C. Chr. (= Hymenophyllum caespitosum Gaudich.).

Hymenophyllum Sm. sect. Pachyloma H. Christ (1897) 15, based on Pachyloma Bosch (1861a) 318, nom. illeg., non DC. (1828) 122, nec Spach (1839) 194. - Type: Hymenophyllum marginatum Hook. \& Grev.

Hymenophyllum Sm. subg. Hemicyatheon Domin (1913) 20. - Hemicyatheon (Domin) Copel. (1938b) 27. - Hymenophyllum Sm. sect. Hemicyatheon (Domin) K. Iwats. (1984) 172. - Type: Hymenophyllum baileyanum Domin.

Hymenophyllum Sm. sect. Acanthotheca Nakai (1926) 262. - Type: Hymenophyllum acanthoides (Bosch) Rosenst.

Hymenophyllum Sm. subg. Buesia C.V. Morton (1932) 336. - Buesia (C.V. Morton) Copel. (1938b) 47. - Hymenophyllum Sm. sect. Buesia (C.V. Morton) C.V. Morton (1968) 164. - Type: Hymenophyllum mirificum C.V. Morton.

Hymenophyllum Sm. subg. Amphipterum C. Presl ex Copel. (1937) 68, based on Amphipterum C. Presl (1849) 258, nom. nud. - Amphipterum (C. Presl ex Copel.) Copel. (1938b) 46. - Hymenophyllum Sm. sect. Amphipterum (C. Presl ex Copel.) C. Chr. ex K. Iwats. (1984) 171. - Type: Hymenophyllum fuscum (Blume) Bosch.

Hymenophyllum Sm. subg. Myriodon Copel. (1937) 73. - Myriodon (Copel.) Copel. (1938b) 47. - Hymenophyllum Sm. sect. Myriodon (Copel.) C.V. Morton (1968) 168. - Type: Hymenophyllum odontophyllum Copel. (= Hymenophyllum brassii C. Chr.).

Rosenstockia Copel. (1947) 36. - Hymenophyllum Sm. subg. Rosenstockia (Copel.) R.M. Tryon \& A.F. Tryon (1981) 134. - Type: Rosenstockia rolandi-principis (Rosenst.) Copel. (= Hymenophyllum rolandi-principis Rosenst.).

Hymenophyllum Sm. sect. Eupectinum Diem \& J.S. Licht. (1959) 635. - Type: Hymenophyllum pectinatum Cav.

Hymenophyllum Sm. sect. Pseudomecodium K. Iwats. (1984) 172. - Pseudomecodium (K. Iwats.) Satou (1997) 269. - Type: Hymenophyllum exsertum (Wall. ex Hook.) Copel.

Hymenophyllum Sm. subg. Euhymenophyllum C. Presl (1843) 31, nom. illeg.; originally includes the lectotype of the genus, Hymenophyllum tunbrigense (L.) Sm.

Hymenophyllum Sm. sect. Serrulata C. Presl (1843) 32, nom. illeg.; originally includes the lectotype of the genus, Hymenophyllum tunbrigense (L.) Sm.

Hymenophyllum Sm. subsect. Evoluta C. Presl (1843) 32, nom. illeg.; originally includes the lectotype of the genus, Hymenophyllum tunbrigense (L.) Sm.

Hymenophyllum Sm. sect. Plumosa K. Iwats. (1984) 172, nom. illeg., non Hymenophyllum Sm. subsect. Plumosa Prantl (1875) 55. - Type: Hymenophyllum levingei C.B. Clarke.

Rhizomes long-creeping, filiform, nearly glabrous or sparsely covered with thick-walled hairs. Stipes to $15 \mathrm{~cm}$ long. Blades simple to quadripinnatifid, narrowly elliptic to subdeltate, 30 by $15 \mathrm{~cm}$, margins of segments entire. Sori usually at the tips of acroscopic segments of pinnae, lips bivalvate, entire or serrate, receptacles included or slightly extruded from involucres.

Distribution - Cosmopolitan; c. 100 species.

Habitat - Usually epiphytic, occasionally epilithic. 
Chromosome base number - Various, $\mathrm{x}=11$ to 28 .

Note - This is the largest subgenus of Hymenophyllum and shows great diversity both in morphology and cytology. Since recent molecular results (Ebihara et al., 2002; Hennequin et al., 2003; Hennequin et al., in press) suggest most of the subgroups included in this subgenus are probably polyphyletic, further study is necessary for the classification within this subgenus.

REPRESENTATIVE SPECIES:

New combinations:

Hymenophyllum trichophorum (Alderw.) Ebihara \& K. Iwats., comb. nov. [based on Trichomanes trichophorum Alderw. (1924) 53, the earlier name for the plant formerly called Amphipterum humatoides Copel. (1941) 465].

Other species:

Hymenophyllum acanthoides (Bosch) Rosenst.; H. armstrongii (Baker) Kirk; H. baileyanum Domin; H. barbatum (Bosch) Baker; H. bivalve (G. Forst.) Sw.; H. blandum Racib.; H. brassii C. Chr.; H. caespitosum Gaudich.; H. cupressiforme Labill.; H. dentatum Cav.; H. denticulatum Sw.; H. deplanchei Mett. ex Kuhn; H. dicranotrichum (C. Presl) Sadeb.; H. dimidiatum Mett. ex Kuhn; H. exsertum Wall. ex Hook.; H. feejeense Brack.; H. foersteri Rosenst.; H. fucoides (Sw.) Sw.; H. fuscum (Blume) Bosch; H. geluense Rosenst.; H. gorgoneum Copel.; H. holochilum (Bosch) C. Chr.; H. johorense Holttum; H. ledermanni Brause; H. levingei C. B. Clarke; H. lobbii T. Moore ex Bosch; H. macrosorum Alderw.; H. marginatum Hook. \& Grev.; H. melanosorum (Copel.) C.V. Morton; H. minimum A. Rich.; H. mirificum C.V. Morton; H. multifidum (G. Forst.) Sw.; H. oligosorum Makino; H. pachydermicum Ces.; H. pectinatum Cav.; H. peltatum (Poir.) Desv.; H. penangianum C.G. Matthew \& H. Christ; H. pumilum C. Moore; H. revolutum Colenso; H. rolandi-principis Rosenst.; H. rubellum Rosenst.; H. rugosum C. Chr. \& Skottsb.; H. secundum Hook. \& Grev.; H. serrulatum (C. Presl) C. Chr.; H. sibthorpioides (Bory ex Willd.) Mett.; H. simonsianum Hook.; H. tenellum (Jacq.) Kuhn; H. triangulare Baker; H. tunbrigense (L.) Sm.; H. walleri Maiden \& Betche; H. wilsonii Hook.

\section{Subgenus Sphaerocionium (C. Presl) C. Chr.}

Hymenophyllum Sm. subg. Sphaerocionium (C. Presl) C. Chr. (1934) 5. - Sphaerocionium C. Presl (1843) 33, t. IV, f. B, t. X, f. B, C. - Hymenophyllum Sm. sect. Sphaerocionium (C. Presl) C. Chr. (1906) XV. - Lectotype: Hymenophyllum hirsutum (L.) Sw. (selected by Copeland (1937) 10).

Trichomanes L. sect. Flabellata C. Presl (1843) 16. - Lectotype: Trichomanes digitatum Sw. (= Hymenophyllum digitatum (Sw.) Fosberg) (selected by Morton (1968) 199).

Hymenophyllum Sm. sect. Pilosa C. Presl (1843) 32. - Lectotype: Hymenophyllum valvatum Hook. \& Grev. (selected by Morton (1968) 168).

Sphaerocionium C. Presl sect. Pilosa C. Presl (1843) 34. - Lectotype: Sphaerocionium schiedeanum C. Presl (= Hymenophyllum crispum Kunth) (selected by Morton (1968) 168).

Sphaerocionium C. Presl sect. Stellata C. Presl (1843) 34. - Lectotype: Sphaerocionium interruptum (Kunze) C. Presl (= Hymenophyllum plumieri Hook. \& Grev.) (selected by Morton (1968) 169).

Gonocormus Bosch sect. Microtrichomanes Mett. ex Prantl (1875) 51, based on Gruppe Microtrichomanes Mett. (1864) 413, nom. nud. - Trichomanes L. sect. Microtrichomanes (Mett. ex Prantl) C. Chr. (1906) XIV. - Microtrichomanes (Mett. ex Prantl) Copel. (1938b) 35. - Crepidomanes (C. Presl) C. Presl subg. Microtrichomanes (Mett. ex Prantl) K. Iwats. (1984) 175. - Lectotype: Gonocormus digitatus (Sw.) Prantl (= Hymenophyllum digitatum (Sw.) Fosberg) (selected by Christensen (1906) XIV).

Hymenophyllum Sm. subsect. Ciliata Prantl (1875) 55. - Lectotype: Hymenophyllum lineare (Sw.) Sw. (selected by Morton (1968) 168).

Hymenophyllum Sm. subsect. Hirsuta Prantl (1875) 55. - Lectotype: Hymenophyllum raddianum Müll.Berol. (= Hymenophyllum fragile (Hedw.) C.V. Morton) (selected by Morton (1968) 170). 
Hymenophyllum Sm. subsect. Plumosa Prantl (1875) 55. - Lectotype: Hymenophyllum plumosum Kaulf. (selected by Morton (1968) 169).

Hymenophyllum Sm. subg. Apteropteris Copel. (1937) 176. - Apteropteris (Copel.) Copel. (1938b) 34. - Hymenophyllum Sm. sect. Apteropteris (Copel.) C.V. Morton (1968) 170. - Sphaerocionium C. Presl subg. Apteropteris (Copel.) K. Iwats. (1984) 173. - Type: Hymenophyllum malingii (Hook.f.) Mett.

Rhizomes long-creeping, filiform, usually covered with stellate hairs. Stipes to $18 \mathrm{~cm}$ long. Blades pinnate to tripinnatifid, elliptic to subdeltate, 165 by $17 \mathrm{~cm}$, covered with stellate hairs, or with simple hairs. Sori at the tips of ultimate segments, bivalvate or tubular, lips entire, receptacles included or slightly extruded from involucres.

Distribution - Cosmopolitan, highly diversified in the Neotropics; c. 70 species.

Habitat - Epiphytic on tree trunks.

Chromosome base number $-\mathrm{x}=36$.

Note - Though the Sphaerocionium species characterized by their stellate hairs have sometimes been treated as an independent genus (e.g., Copeland, 1938b; Iwatsuki, 1984), we reduced them to a subgenus of Hymenophyllum for the reason noted in the description of the genus. Our present definition of Sphaerocionium also includes most of the Microtrichomanes species (Ebihara et al., 2004) whose hairs have been transformed from stellate into simple. Although Morton (1947) subdivided this group chiefly by the nature and placement of the hairs, recent studies (Ebihara et. al., 2004, Hennequin et al., in press) suggest their phylogenetic relationships are more closely related to their geographical distributions.

\section{REPRESENTATIVE SPECIES:}

Hymenophyllum adiantoides Bosch; H. aeruginosum (Poir.) Carmich.; H. amabile C.V. Morton; H. angustum Bosch; H. antillense (Jenman) Jenman; H. braithwaitei Ebihara \& K. Iwats.; H. capillare Desv.; H. consanguineum C.V. Morton; H. crispum Kunth; H. dependens C.V. Morton; H. digitatum (Sw.) Fosberg; H. elegans A. Spreng.; H. elegantulum Bosch; H. ferrugineum Colla; H. fragile (Hedw.) C.V. Morton; H. frankliniae Colenso; H. fusugasugense H. Karst. ex Sturm; H. glaziovii Baker; H. hemipteron Rosenst.; H. hirsutum (L.) Sw.; H. hirtellum Sw.; H. horizontale C.V. Morton; H. karstenianum Sturm; H. lanatum Fée; H. lanceolatum Hook. \& Arn.; H. lindenii Hook.; H. lineare (Sw.) Sw.; H. lobato-alatum Klotzsch; H. lyallii Hook.f.; H. malingii (Hook.f.) Mett.; H. microcarpum Desv.; H. molle C.V. Morton; H. multialatum C.V. Morton; H. nitidulum (Bosch) Ebihara \& K. Iwats.; H. obtusum Hook. \& Arn.; H. palmatifidum (Müll. Berol.) Ebihara \& K. Iwats.; H. pilosissimum C. Chr.; H. plumieri Hook. \& Grev.; H. plumosum Kaulf.; H. pulchellum Schltdl. \& Cham.; H. pyramidatum Desv.; H. roraimense C.V. Morton; H. ruizianum (Klotzsch) Kunze; H. semiglabrum Rosenst.; H. sericeum (Sw.) Sw.; H. sieberi (C. Presl) Bosch; H. simplex C.V. Morton; H. speciosum Bosch; H. splendidum Bosch; H. subobtusum Rosenst.; H. subrigidum H. Christ; H. tomaniiviense (Brownlie) Ebihara \& K. Iwats.; H. tomentosum Kunze; H. trapezoidale Liebm.; H. trichophyllum Kunth; H. urbanii Brause; H. valvatum Hook. \& Grev.; H. verecundum C.V. Morton.

\section{Subgenus Mecodium C. Presl ex Copel.}

Hymenophyllum Sm. subg. Mecodium C. Presl ex Copel. (1937) 93, based on Mecodium C. Presl (1849) 258, nom. nud. - Mecodium (C. Presl ex Copel.) Copel. (1938b) 17. - Lectotype: Hymenophyllum polyanthos (Sw.) Sw. (selected by Copeland (1937) 10).

Hymenophyllum Sm. sect. Glabra Prantl (1875) 54. — Lectotype: Hymenophyllum polyanthos (Sw.) Sw. (selected by Morton (1968) 172).

Hymenophyllum Sm. sect. Corrugatae K. Iwats. (1984) 173. - Type: Hymenophyllum ooides F. Muell. \& Baker. 
Rhizomes long-creeping, filiform, nearly glabrous. Stipes up to $10 \mathrm{~cm}$ long. Blades pinnate to tripinnatifid, elliptic to subdeltate, 45 by $6 \mathrm{~cm}$, margins of segments entire. Sori at the tips of ultimate segments, lips bivalvate, entire, receptacles included in involucres.

Distribution - Cosmopolitan; more than 35 species.

Habitat - Epiphytic on tree trunks.

Chromosome base number $-\mathrm{x}=28$.

Note - This subgenus corresponds to the cosmopolitan species $H$. polyanthos and its local derivatives; it also corresponds to the ' $H$. polyanthos clade' in Hennequin et al. (in press). The chromosome base number of $\mathrm{x}=28$ is a synapomorphy of this subgenus. Although Presl (1849) originally used the generic name Mecodium for $H$. sanguinolentum, which is now referred to subg. Myrmecostylum, Copeland (1937) selected $H$. polyanthos as the type when he legitimized the name - the type designation may not be correctable even it was later corrected to $H$. sanguinolentum by Copeland himself (1938b).

REPRESENTATIVE SPECIES:

New combinations:

Hymenophyllum novoguineense (Rosenst.) K. Iwats., comb. nov. [based on Hymenophyllum blumeanum A. Spreng. var. novoguineense Rosenst. (1908b) 371].

Other species:

Hymenophyllum abruptum Hook.; H. apiculatum Mett. ex Kuhn; H. axillare Sw.; H. brevifrons Kunze; H. copelandii C.V. Morton; H. corrugatum H. Christ; H. cuneatum Kunze; H. darwinii Hook.; H.fendlerianum Sturm; H. fumarioides Bory ex Willd.; H. inaequale (Poir.) Desv.; H. kuhnii C. Chr.; H. mnioides Baker; H. myriocarpum Hook.; H. ooides F. Muell. \& Baker; H. paniculiflorum C. Presl; H. polyanthos (Sw.) Sw.; H. rarum R. Br.; H. recurvum Gaudich.; H. siliquosum H. Christ; H. undulatum Sw.; H. whitei Goy; H. wrightii Bosch.

4. Subgenus Globosa (Prantl) Ebihara \& K. Iwats., comb. nov.

Based on Hymenophyllum Sm. sect. Globosa Prantl (1875) 55. - Lectotype: Hymenophyllum junghuhnii Bosch (selected by Morton (1968) 172).

Sphaerocionium C. Presl sect. Glabra C. Presl (1843) 34, non Hymenophyllum Sm. sect. Glabra Prantl (1875) 54. - Lectotype: Hymenophyllum caudiculatum Mart. (selected by Morton (1968) 172).

Rhizomes long-creeping, filiform to wiry, nearly glabrous. Stipes to $15 \mathrm{~cm}$ long. Blades tri- to quadripinnate, elliptic to subdeltate, 60 by $15 \mathrm{~cm}$, margin of segments usually entire, minutely serrate in some species. Sori at the tips of ultimate segments, lips bivalvate, entire or serrate, receptacles included in involucres, often capitate.

Distribution - Tropics to temperate regions of Asia and Pacific areas; at least one species is distributed in the southern part of South America; c. 25 species.

Habitat - Epiphytic, or occasionally epilithic.

Chromosome base number $-\mathrm{x}=36$.

Note - This subgenus corresponds to the ' $H$. australe clade' in Hennequin et al. (in press). It has been confused with the H. polyanthos group (subg. Mecodium in the present system), but features of the rhizome (e.g., dorsiventral protostele) are clearly different (Hennequin et al., in press). The earliest name given to this group, 'Sphaerocionium sect. Glabra' (Presl, 1843) becomes a later homonym of Hymenophyllum sect. 
Glabra (Prantl, 1875) when it is placed under Hymenophyllum according to the latest Code (Greuter et al., 2000, Art. 21.3, Note 1). Therefore the name of this subgenus should be Globosa.

\section{REPRESENTATIVE SPECIES:}

Hymenophyllum australe Willd.; H. badium Hook. \& Grev.; H. caudiculatum Mart.; H. demissum (G. Forst.) Sw.; H. emarginatum Sw.; H. fimbriatum J. Sm. ex Hook.; H. flexuosum A. Cunn.; H. imbricatum Blume; H. javanicum A. Spreng.; H. junghuhnii Bosch; H. longifolium Alderw.; $H$. productum Kunze; H. reinwardtii Bosch; H. riukiuense $\mathrm{H}$. Christ; H. salakense Racib.; H. thuidium Harr.; H. treubii Racib.

\section{Subgenus Pleuromanes (C. Presl) Ebihara \& K. Iwats., comb. nov.}

Based on Trichomanes L. subg. Pleuromanes C. Presl (1849) 17. - Pleuromanes (C. Presl) C. Presl (1849) 258. - Crepidomanes (C. Presl) C. Presl subg. Pleuromanes (C. Presl) K. Iwats. (1984) 174. - Type: Trichomanes acutum C. Presl (1843) 42 (= Hymenophyllum acutum (C. Presl) Ebihara \& K. Iwats., comb. nov., non Hymenophyllum acutum Mett., nom. nud.).

Craspedoneuron Bosch (1861a) 322. - Trichomanes L. sect. Craspedoneuron (Bosch) Prantl (1875) 52. - Type: Trichomanes album Blume (= Hymenophyllum pallidum (Blume) Ebihara \& K. Iwats., comb. nov. [based on Trichomanes pallidum Blume (1828) 225]).

Rhizomes long-creeping, filiform, sparsely covered with whitish/yellowish hairs. Stipes to $12 \mathrm{~cm}$ long. Blades bi- to tripinnate, elliptic to subdeltate, 35 by $6 \mathrm{~cm}$, covered with whitish/yellowish multicellular hairs or almost glabrous, margins of segments entire. Sori at the tips of ultimate segments, lips slightly to distinctly bivalvate, entire, receptacles included or slightly exserted from involucres.

Distribution - Old World tropics, especially in the Pacific region; 5 species.

Habitat - Epiphytic on tree trunks (including tree ferns).

Chromosome base number $-\mathrm{x}=36$.

Note - This subgenus contains H.flabellatum plus its local forms and Pleuromanes, formerly considered a member of Trichomanes s.l.; it also corresponds to ' $\mathrm{H}$. flabellatum clade' in Hennequin et al. (in press).

\section{REPRESENTATIVE SPECIES:}

Hymenophyllum acutum (C. Presl) Ebihara \& K. Iwats.; H. flabellatum Labill.; H. leratii Rosenst.; H. pallidum (Blume) Ebihara \& K. Iwats.; H. rufescens Kirk.

\section{Subgenus Myrmecostylum (C. Presl) Ebihara \& K. Iwats., comb. nov.}

Based on Myrmecostylum C. Presl (1843) 27, t. X, f. A. - Hymenophyllum Sm. sect. Myrmecostylum (C. Presl) C. Chr. (1906) XVI. - Lectotype: Myrmecostylum tortuosum (Hook. \& Grev.) C. Presl (= Hymenophyllum tortuosum Hook. \& Grev.) (selected by Christensen (1906) XVI).

Ptychophyllum C. Presl (1843) 28, t. XI, f. E. - Hymenophyllum Sm. sect. Ptychophyllum (C. Presl)

C. Chr. (1906) XVI. - Type: Ptychophyllum plicatum (Kaulf.) C. Presl (= Hymenophyllum plicatum Kaulf.).

Tetralasma Phil. (1860) 208. - Type: Hymenophyllum quadrifidum Phil. (= Hymenophyllum plicatum Kaulf.), no generic combination has been made for this genus.

Rhizomes long-creeping, filiform to wiry, sparsely covered with thick-walled hairs. Stipes to $15 \mathrm{~cm}$ long. Blades pinnatifid to quadripinnate, elliptic to subdeltate, 55 by $14 \mathrm{~cm}$, margins of segments entire or serrate. Sori at the tips of ultimate segments, lips bivalvate, entire or serrate, receptacles included in involucres. 
Distribution - Southern Chile, New Zealand and New Caledonia; at least 8 species are known.

Habitat - Epiphytic on tree trunks, occasionally terrestrial.

Chromosome base number $-\mathrm{x}=36$.

Note - This subgenus corresponds to the ' $H$. sanguinolentum clade' in Hennequin et al. (in press), and is characterized by brown multicellular hairs on the stipes or blade margins, or alternatively by dentate blade margins. In exceptional cases, $H$. sanguinolentum has nearly glabrous fronds, almost indistinguishable from $H$. polyanthos - but containing of oils that make an impression of the blades on the mounting paper; this feature is a characteristic of this group.

REPRESENTATIVE SPECIES:

Hymenophyllum krauseanum Phil.; H. magellanicum (Desv.) Willd.; H. paninese Ebihara \& K. Iwats.; H. plicatum Kaulf.; H. sanguinolentum (G. Forst.) Sw.; H. scabrum A. Rich.; H. tortuosum Hook. \& Grev.; H. villosum Colenso.

\section{Subgenus Hymenoglossum (C. Presl) R.M. Tryon \& A.F. Tryon}

Hymenophyllum Sm. subg. Hymenoglossum (C. Presl) R.M. Tryon \& A.F. Tryon (1981) 134. - Hymenoglossum C. Presl (1843) 35. - Hymenophyllum Sm. sect. Hymenoglossum (C. Presl) T. Moore (1857) cxii. - Type: Hymenoglossum cruentum (Cav.) C. Presl (= Hymenophyllum cruentum Cav.).

Hymenophyllum Sm. sect. Integra C. Presl (1843) 32. - Lectotype: Hymenophyllum asplenioides Sw. (selected by Morton (1968) 172).

Rhizomes long-creeping, filiform, nearly glabrous. Stipes $7-15 \mathrm{~cm}$ long. Blades simple to pinnatifid, ovate to narrowly ovate, 15 by $4 \mathrm{~cm}$, margins of segments entire. Sori at the frond margins, slightly immersed in the laminae, lips bivalvate, entire, receptacles included in involucres.

Distribution - Central to South America and Madagascar; 3 species known.

Habitat - Epiphytic on tree trunks.

Chromosome base number $-\mathrm{x}=36$ ?

Note - Hennequin et al. (in press) revealed that $H$. heimii of Madagascar probably has a sister relationship to $H$. cruentum of southern Chile (formerly treated as a monotypic genus, Hymenoglossum). A Central-South American species, H. asplenioides, is also placed here because of its morphological resemblance to the two species above.

REPRESENTATIVE SPECIES:

Hymenophyllum asplenioides Sw.; H. cruentum Cav.; H. heimii Tardieu.

\section{Subgenus Fuciformia Ebihara \& K. Iwats., subg. nov.}

A subgenere Globosa rhizomatibus brevibus et erectis differt.

Based on Hymenophyllum Sm. sect. Fuciformia H. Christ (1897) 20, nom. nud. - Type: Hymenophyllum fuciforme $\mathrm{Sw}$.

Rhizomes erect or short-creeping, thick, densely covered with reddish hairs. Stipes 3-15 $\mathrm{cm}$ long. Blades tri- to quadripinnate, ovate to narrowly ovate, 65 by $15 \mathrm{~cm}$, margins of segments entire. Sori at the tips of ultimate segments, lips bivalvate, entire or wavy, receptacles included or slightly extruded from involucres. 
Distribution - Southern temperate regions (Chile and New Zealand); 2 species.

Chromosome base number $-\mathrm{x}=36$.

Habitat - Epiphytic, epilithic or terrestrial.

Note - This subgenus comprises only two species having tufted rhizomes, an exceptional feature in Hymenophyllum. Evidence suggests that these two species, which are disjunctly distributed in southern Chile and New Zealand, form a monophyletic group (Hennequin et al., in press).

REPRESENTATIVE SPECIES:

Hymenophyllum fuciforme Sw.; H. pulcherrimum Colenso.

\section{Subgenus Diploöphyllum (Bosch) Ebihara \& K. Iwats., comb. nov.}

Based on Diploöphyllum Bosch (1861a) 322, non Diplophyllum (Dumort.) Dumort. (1835) 15, nom. cons., ICBN 2000. - Hymenophyllum Sm. sect. Diploöphyllum C. Chr. (1906) XV. - Hymenophyllum Sm. subsect. Diploöphyllum (C. Chr.) C.V. Morton (1968) 174, as 'Diplophyllum'. - Lectotype and sole species (in the present treatment): Hymenophyllum dilatatum (G. Forst.) Sw. (selected by Christensen (1906) XV).

Rhizomes long-creeping, wiry, sparsely covered with dark hairs. Stipes $2-15 \mathrm{~cm}$ long. Blades tri- to quadripinnate, ovate or narrowly ovate, 55 by $15 \mathrm{~cm}$, laminae more than two cells thick, margins of segments entire. Sori at the tips of ultimate segments, lips bivalvate, entire, receptacles included in involucres.

Distribution - New Zealand, monotypic.

Habitat - Epiphytic on tree trunks.

Chromosome base number $-\mathrm{x}=36$.

Note - This monotypic subgenus is characterized by multilayered laminar cells throughout its blades and long-creeping rhizomes. Molecular data (Hennequin et al., in press) suggest this is closely related to the subgenera Fuciformia and Cardiomanes. Van den Bosch's (1861a) original spelling of this taxon was Diploöphyllum, neither 'Diploophyllum' as used by Copeland (1938b) nor 'Diplophyllum' by Morton (1968), and its inclusion of a diacritical sign as a diaeresis is legitimate in the latest Code (Greuter et al., 2000, Art. 60.6).

\section{Subgenus Cardiomanes (C. Presl) Ebihara \& K. Iwats., comb. nov.}

Based on Cardiomanes C. Presl (1843) 12. - Trichomanes L. subg. Cardiomanes (C. Presl) H. Christ (1897) 33. - Type and sole species: Trichomanes reniforme G. Forst. (1786) 84 (= Hymenophyllum nephrophyllum Ebihara \& K. Iwats., nom. nov. = Cardiomanes reniforme (G. Forst.) C. Presl, non Hymenophyllum reniforme Hook.).

Rhizomes long-creeping, wiry, sparsely covered with dark hairs. Stipes $5-25 \mathrm{~cm}$ long. Blades simple, kidney-shaped, 35 by $13 \mathrm{~cm}$, laminae more than two cells thick, margins of segments entire. Sori at the blade margins, tubular, lips entire, receptacles exserted from involucres.

Distribution - New Zealand, monotypic.

Habitat - Epiphytic.

Chromosome base number $-\mathrm{x}=36$.

Note - One of the most distinct subgenera of Hymenophyllum (formerly included in Trichomanes s.l. due to its cup-shaped involucres). A replacement name is necessary when Cardiomanes reniforme is moved to Hymenophyllum. 


\section{DIDYMOGLOSSUM}

Didymoglossum Desv. (1827) 330. - Trichomanes L. sect. Didymoglossum (Desv.) T. Moore (1857) cx. - Trichomanes L. subg. Didymoglossum (Desv.) C. Chr. (1906) XIV. - Lectotype: Didymoglossum muscoides (Sw.) Desv. (= Didymoglossum hymenoides (Hedw.) Copel.) (selected by Christensen (1906) XIV, as 'Trichomanes hymenoides Hedw.').

Distribution - Throughout the tropics; more than 30 species.

Chromosome base number $-\mathrm{x}=34$.

Note - Corresponding to the Di clade in Ebihara et al. (submitted), and also to Morton's (1968) Trichomanes subgenus Didymoglossum. This genus contains three genera sensu Copeland (1938b): Didymoglossum, Microgonium and Lecanium (Lecanolepis). All species in Didymoglossum are dwarf epiphytes, mainly in tropical regions, and false veinlets are present. Though both Copeland (1938b) and Morton (1968) used the presence of marginal setae as the primary character to distinguish Microgonium from Didymoglossum, there was evidently secondary loss of marginal hairs in some 'Microgonium' species (Ebihara et al., submitted). Therefore, submarginal false veinlets stand as the primary character to separate the two subgenera.

\section{KEY TO THE SUBGENERA}

1a. Submarginal false veinlets absent . . . . . . . 1. Subg. Didymoglossum

b. Submarginal false veinlets present .......... 2. Subg. Microgonium

\section{Subgenus Didymoglossum}

Hemiphlebium C. Presl sect. Lecanium Prantl (1875) 46, based on Lecanium C. Presl (1843) 11, t. I, nom. illeg., non Reinw. (1825) 48. - Trichomanes L. sect. Lecanium (Prantl) H. Christ (1897) 25. - Lecanolepis Pic.Serm. (1973) 449. - Type: Lecanium membranaceum (L.) C. Presl (= Didymoglossum membranaceum (L.) Vareschi).

Didymoglossum Desv. sect. Flabellata C. Presl (1843) 23. - Lectotype: Didymoglossum sphenoides (Kunze) C. Presl (= Didymoglossum punctatum (Poir.) Desv.) (selected by Morton (1968) 191).

Didymoglossum Desv. sect. Pinnata C. Presl (1843) 23. - Lectotype: Didymoglossum muscoides (Sw.) Desv. (= Didymoglossum hymenoides (Hedw.) Copel.) (selected by Morton (1968) 191).

Hemiphlebium C. Presl (1843) 25, t. IX. - Trichomanes L. sect. Hemiphlebium (C. Presl) T. Moore (1857) cx. - Trichomanes L. subg. Hemiphlebium (C. Presl) H. Christ (1897) 23. - Type: Hemiphlebium pusillum (Sw.) C. Presl (=Didymoglossum pusilum (Sw.) Desv.).

Rhizomes long-creeping, frequently branching, usually filiform, to $0.5 \mathrm{~mm}$ diam., densely covered with dark-coloured hairs, protostele collateral to regressed, cortex petiole-like, roots absent, root-like shoots present (sensu Schneider, 2000). Stipes often quite reduced, up to $1 \mathrm{~cm}$, at a distance from the adjacent ones. Blades usually simple, or sometimes lobed to pinnatifid, elliptic to narrowly ovate, 6 by $6 \mathrm{~cm}$, venation catadromous, often flabelliform, submarginal false veinlets absent, longitudinal false veinlets parallel to the true veins present, internal cell walls thin and straight, setae or scale-like projections sometimes. Sori epitactic or pantotactic, often immersed in the laminae, campanulate, lips bilabiate or occasionally truncate, often dark-margined, receptacles exserted.

Distribution - Throughout the tropics, mainly in the New World; more than 20 species. 
Habitat - Epilithic or low-epiphytic.

Note - Corresponding to the Le subclade in Ebihara et al. (submitted).

REPRESENTATIVE SPECIES:

New combinations:

Didymoglossum hildebrandtii (Kuhn) Ebihara \& Dubuisson, comb. nov. [based on Trichomanes hildebrandtii Kuhn (1879) 70]; D. lorencei (Tardieu) Ebihara \& Dubuisson, comb. nov. [based on Trichomanes lorencei Tardieu (1977) 147]; D. motleyi (Bosch) Ebihara \& K. Iwats., comb. nov. [based on Trichomanes motleyi Bosch (1861b) 145]; D. sublimbatum (Müll.Berol.) Ebihara \& K. Iwats., comb. nov. [based on Trichomanes sublimbatum Müll. Berol. (1854) 737]; D. tahitense (Nadeaud) Ebihara \& K. Iwats., comb. nov. [based on Trichomanes tahitense Nadeaud (1873) 18].

Other species:

Didymoglossum angustifrons Fée; D. curtii (Rosenst.) Pic.Serm.; D. exiguum (Bedd.) Copel.; D. gourlianum (Grev. ex J. Sm.) Pic.Serm.; D. hymenoides (Hedw.) Copel.; D. krausii (Hook. \& Grev.) C. Presl; D. liberiense (Copel.) Copel.; D. lineolatum Bosch; D. melanopus (Baker) Copel.; D. membranaceum (L.) Vareschi; D. nummularium Bosch; D. ovale E. Fourn.; D. petersii (A. Gray) Copel.; D. pinnatinervium (Jenman) Pic.Serm.; D. punctatum (Poir.) Desv.; D. pusillum (Sw.) Desv.; D. reptans (Sw.) C. Presl; D. rhipidophyllum (Sloss.) Pic.Serm.

\section{Subgenus Microgonium (C. Presl) Ebihara \& K. Iwats., comb. nov.}

Based on Microgonium C. Presl (1843) 19, t. VI, f. A, B. - Hemiphlebium C. Presl sect. Microgonium (C. Presl) Prantl (1875) 46. - Trichomanes L. sect. Microgonium (C. Presl) H. Christ (1897) 24. - Lectotype: Microgonium cuspidatum (Willd.) C. Presl (= Didymoglossum cuspidatum (Willd.) Ebihara \& Dubuisson, comb. nov. [based on Trichomanes cuspidatum Willd. (1810) 499]) (selected by Christensen (1906) XIV).

Rhizomes long-creeping, frequently branching, filiform, to $0.5 \mathrm{~mm}$ diam., densely covered with dark hairs, protostele collateral to regressed (see Ebihara et al., submitted), cortex petiole-like, roots absent, root-like shoots present. Stipes often quite reduced, to $1 \mathrm{~cm}$, at a distance from the adjacent ones. Blades usually simple, or sometimes lobed to pinnatifid, elliptic to narrowly ovate, 3 by $2 \mathrm{~cm}$, venation catadromous, continuous submarginal false veinlets present, longitudinal false veinlets parallel to the true veins present, internal cell walls thin and straight, margins entire and glabrous. Sori epitactic or pantotactic, often immersed in the laminae, tubular, lips often dilate, receptacles exserted.

Distribution - Throughout the tropics, mainly in the Old World; more than 10 species.

Habitat - Epilithic or low-epiphytic.

Note - Corresponding to the Mg subclade in Ebihara et al. (submitted).

REPRESENTATIVE SPECIES:

New combinations:

Didymoglossum bimarginatum (Bosch) Ebihara \& K. Iwats., comb. nov. [based on Microgonium bimarginatum Bosch (1861b) 143]; D. ekmanii (Wess. Boer) Ebihara \& Dubuisson, comb. nov. [based on Trichomanes ekmanii Wess. Boer (1962) 319, f. 33a, b]; D. godmanii (Hook.) Ebihara \& Dubuisson, comb. nov. [based on Trichomanes godmanii Hook. in Baker (1866) 337, t. 8, f. A]; D. kapplerianum (Sturm) Ebihara \& Dubuisson, comb. nov. [based on Trichomanes kapplerianum Sturm in Martius (1859) 276]; D. kirkii (Hook.) Ebihara \& Dubuisson, comb. nov. [based on Trichomanes kirkii Hook. in Hook. \& Baker (1867) 78]; D. lenormandii (Bosch) Ebihara \& Dubuisson, comb. nov. [based on Trichomanes lenormandii Bosch (1861b) 144]; D. mindorense (H. Christ) K. Iwats., comb. nov. [based on Trichomanes mindorense H. Christ (1908a) 270]; D. pygmaeum (C. Chr.) Ebihara \& Dubuisson, comb. nov. [based on Trichomanes pygmaeum C. Chr. (1920) 10, f. 2]; D. sinuatum (Bonap.) 
Ebihara \& Dubuisson, comb. nov. [based on Trichomanes sinuatum Bonap. (1920) 25]; D. wesselsboeri Ebihara \& Dubuisson, nom. nov. [based on Trichomanes hookeri C. Presl (1843) 16, non Didymoglossum hookeri C. Presl].

Other species:

Didymoglossum cuspidatum (Willd.) Ebihara \& Dubuisson.

\section{CREPIDOMANES}

Crepidomanes (C. Presl) C. Presl (1849) 258. - Trichomanes L. subg. Crepidomanes C. Presl (1849) 17. - Trichomanes L. sect. Crepidomanes (C. Presl) Prantl (1875) 51. - Type: Trichomanes intramarginale Hook. \& Grev. (= Crepidomanes intramarginale (Hook. \& Grev.) C. Presl).

Distribution - Throughout the Old World tropics to northern temperate regions; more than 30 species.

Chromosome base number $-\mathrm{x}=36$.

Note - This genus corresponds to the PT clade in Ebihara et al. (submitted), and also to Copeland's (1938b) genera Crepidomanes, Gonocormus, Crepidopteris (Reediella) p.p., Vandenboschia p.p. and Microtrichomanes p.p. Copeland's (1938b) genera Crepidomanes and Gonocormus are placed here, and a part of Crepidopteris, Vandenboschia and Microtrichomanes are also included. Most species of Crepidomanes are epiphytes, mainly in rain forests of the Paleotropics; however, Nesopteris, with a short, erect rhizomes, is also included here.

\section{KEY TO THE SUBGENERA AND SECTIONS}

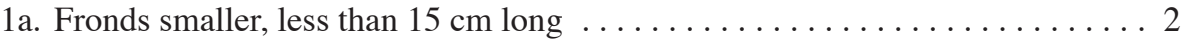

b. Fronds larger, more than $15 \mathrm{~cm}$ long $\ldots \ldots \ldots \ldots \ldots$ 2. Subg. Nesopteris

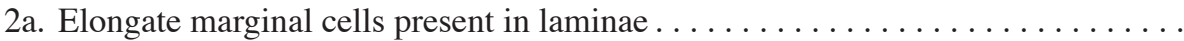

1c. Subg. Crepidomanes sect. Crepidium

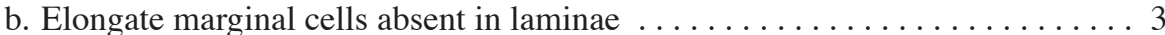

3a. Proliferation present on stipes ... . 1b. Subg. Crepidomanes sect. Gonocormus

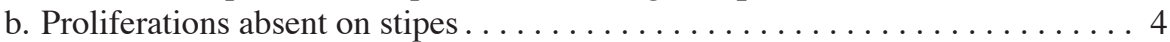

4a. Blades flabellate. . . . . . . . . . 1b. Subg. Crepidomanes sect. Gonocormus

b. Blades pinnate ........... 1a. Subg. Crepidomanes sect. Crepidomanes

\section{Subgenus Crepidomanes}

Distribution - Throughout the Paleotropics.

Note - Corresponding to the $\mathrm{Cr}$ subclade in Ebihara et al. (submitted). Three sections are recognized in this subgenus.

\section{1a. Section Crepidomanes}

Trichomanes L. subg. Minora Prantl (1875) 51. - Lectotype: Trichomanes intramarginale Hook. \& Grev. (= Crepidomanes intramarginale (Hook. \& Grev.) Copel.) (selected by Morton (1968) $181)$.

Trichomanes L. sect. Taschneria C. Presl ex C. Chr. (1906) XV, based on Taschneria C. Presl (1849) 258, nom. nud. - Crepidomanes (C. Presl) C. Presl sect. Taschneria (C. Presl ex C. Chr.) K. Iwats. (1984) 175. - Type:Trichomanes filicula Bory ex Willd. (=Crepidomanes bipunctatum (Poir.) Copel.). 
Rhizomes long-creeping, frequently branching, filiform, $0.1-0.5 \mathrm{~mm}$ diam., densely covered with dark hairs, protostele subcollateral, cortex with centrifuge sclerification, roots absent, root-like shoots present. Stipes sometimes reduced, or to $8 \mathrm{~cm}$ long, at a distance from the adjacent ones. Blades simple to quadripinnatifid, elliptic to subdeltate, 12 by $6 \mathrm{~cm}$, venation anadromous, false veinlets often present, submarginally (continuous or interrupted) and/or parallel (but not connected) to true veins, laminae almost always one cell thick, exceptionally thicker $(C$. intramarginale), internal cell walls thin and straight. Sori paratactic, tubular, lips usually bilabiate, often deltate, sometimes dilate, receptacles exserted.

Distribution - Throughout the Paleotropics; more than 20 species.

Habitat - Usually epilithic on wet rocks along streams, sometimes epiphytic on tree trunks.

Note - Crepidomanes in the strict sense contains only those species that have false veinlets, but recent study (Ebihara et al., submitted) has shown that some species lacking false veinlets (formerly placed under Vandenboschia by Copeland) are closely related to species that have them and/or are nested in a clade consisting of species with false veinlets.

\section{REPRESENTATIVE SPECIES:}

New combinations:

Crepidomanes africanum (H. Christ) Ebihara \& Dubuisson, comb. nov. [based on Trichomanes africanum H. Christ (1909a) 21]; C. chevalieri (H. Christ) Ebihara \& Dubuisson, comb. nov. [based on Trichomanes chevalieri H. Christ (1908b) 106]; C. draytonianum (Brack.) Ebihara \& K. Iwats., comb. nov. [based on Trichomanes draytonianum Brack. (1854) 252, pl. 36, f. 3]; C. fallax (H. Christ) Ebihara \& Dubuisson, comb. nov. [based on Trichomanes fallax H. Christ (1909b) 24]; C. mettenii (C. Chr.) Ebihara \& Dubuisson, comb. nov. [based on Trichomanes mettenii C. Chr. (1906) 644].

\section{Other species:}

Crepidomanes barnardianum (F.M. Bailey) Tindale; C. bipunctatum (Poir.) Copel.; C. christii (Copel.) Copel.; C. clarenceanum (F. Ballard) Pic. Serm.; C. frappieri (Cordem.) J.P. Roux; C. inopinatum (Pic.Serm.) J.P. Roux; C. intramarginale (Hook. \& Grev.) Copel.; C. kurzii (Bedd.) Tagawa \& K. Iwats.; C. latealatum (Bosch) Copel.; C. latemarginale (D.C. Eaton) Copel.; C. melanotrichum (Schltdl.) J.P. Roux; C. rupicolum (Racib.) Copel.; C. schmidtianum (Zenker ex Taschner) K. Iwats.; C. vitiense (Baker) Bostock.

\section{1b. Section Gonocormus (Bosch) K. Iwats.}

Crepidomanes (C. Presl) C. Presl sect. Gonocormus (Bosch) K. Iwats. (1984) 174. - Gonocormus Bosch (1861a) 321. - Trichomanes L. sect. Gonocormus (Bosch) H. Christ (1897) 27. - Trichomanes L. subg. Gonocormus (Bosch) C. Chr. (1906) XIV. - Lectotype: Gonocormus prolifer (Blume) Prantl (= Crepidomanes minutum (Blume) K. Iwats.) (selected by Christensen (1906) XIV).

Rhizomes long-creeping, frequently branching, filiform, c. $0.15 \mathrm{~mm}$ diam., covered with dark brown hairs or glabrescent, protostele subcollateral, cortex with centrifugal sclerification, roots absent, root-like shoots present. Stipes very short, or to $3 \mathrm{~cm}$ long, at a distance from the adjacent ones, proliferations often observed. Blades simple to quadripinnatifid, flabellate to narrowly ovate, 12 by $3 \mathrm{~cm}$, venation anadromous, false veinlets absent, internal cell walls thin and straight. Sori paratactic or pantotactic, campanulate, lips dilate, receptacles long-exserted. 
Distribution - Throughout the Paleotropics; 1 polymorphic species, Crepidomanes minutum (Blume) K. Iwats., is recognized here.

Habitat - Epilithic or epiphytic on tree trunks.

Note - The single species ranges from Africa to the Pacific according to Yoroi \& Iwatsuki (1977). There is much genetic variation in the $r b c L$ sequences of this species from various localities, but it corresponds neither to morphological features nor to geographical distribution (A. Ebihara, unpublished data). Further studies are needed.

1c. Section Crepidium (C. Presl) Ebihara \& K. Iwats., comb. nov.

Based on Didymoglossum Desv. subg. Crepidium C. Presl (1843) 23. - Trichomanes L. sect. Crepidium (C. Presl) C. Chr. (1906) XV. - Crepidopteris Copel. (1938b) 57, nom. illeg., non Benth. (1862). - Crepidophyllum C.F. Reed (1948) 88, nom. illeg., non Herzog (1926) 356. — Reediella Pic.Serm. (1970) 719. - Crepidomanes (C. Presl) C. Presl subg. Crepidium (C. Presl) K. Iwats. (1984) 174. - Type: Didymoglossum humile (G. Forst.) C. Presl (= Crepidomanes humile (G. Forst.) Bosch).

Rhizomes long-creeping, frequently branching, filiform, 0.3-0.4 mm diam., densely covered with dark brown or blackish hairs, protostele subcollateral, cortex with centrifugal sclerification, roots absent, root-like shoots present. Stipes $0.2-1.2 \mathrm{~cm}$ long, at a distance from the adjacent ones. Blades bipinnatifid to tripinnatifid, ovate to narrowly ovate, $1-8$ by $2-3 \mathrm{~cm}$ wide, venation anadromous, double rows of elongate marginal cells present, false veinlets absent, internal cell walls thin and straight. Sori paratactic, tubular, lips dilate, receptacles long-exserted.

Distribution - Old World tropics (Asia to Pacific); at least 1 species, Crepidomanes humile (G. Forst.) Bosch.

Habitat - Epilithic or low-epiphytic.

Note - Crepidium (Reediella), a group characterized by marginal elongated cell rows, has been shown to be an unnatural group (Ebihara et al., submitted) - species having single rows of elongated cells are attributed to another genus, Polyphlebium, in the present system. As a result, this section contains only $C$. humile.

\section{Subgenus Nesopteris (Copel.) Ebihara \& K. Iwats., comb. nov.}

Based on Nesopteris Copel. (1938b) 65. - Trichomanes L. sect. Nesopteris (Copel.) C.V. Morton (1968) 190. - Cephalomanes C. Presl subg. Nesopteris (Copel.) K. Iwats. (1984) 176. - Type: Nesopteris grandis (Copel.) Copel. (= Crepidomanes grande (Copel.) Ebihara \& K. Iwats., comb. nov. [based on Trichomanes grande Copel. (1911) 70].

Rhizomes suberect, ascending or long-creeping, quite thick, 2.8-3.5 mm diam., densely covered with dark hairs, protostele massive, cortex homogeneous, roots numerous and robust. Stipes to $25 \mathrm{~cm}$ long, very close to each other except in C.aphlebioides. Blades 4- or 5-pinnate, elliptic to narrowly ovate, $15-50(-70)$ by $10-20(-25) \mathrm{cm}$, venation anadromous, false veinlets absent, internal cell walls thin and straight. Sori paratactic, tubular, lips often dilate, receptacles long-exert.

Distribution - Old World tropics (Asia to Pacific); at least 4 species.

Habitat - Terrestrial or epilithic, occasionally climbing tree trunks.

Note - Large fronds and stout rhizomes observed in members of this subgenus are apparently quite different from those of subgenus Crepidomanes. Our study (Ebihara 
et al., submitted) revealed that the erect rhizomes of the Nesopteris species are probably the result of an exceptional evolution from creeping rhizomes to erect ones, which is in the direction opposite to the evolutional trait of Hymenophyllaceae. We assume that C. aphlebioides, the only species of this subgenus having long-creeping rhizomes, is transitional between the two subgenera.

REPRESENTATIVE SPECIES:

New combinations:

Crepidomanes intermedium (Bosch) Ebihara \& K. Iwats., comb. nov. [based on Trichomanes intermedium Bosch (1861b) 179]; C. thysanostomum (Makino) Ebihara \& K. Iwats., comb. nov. [based on Trichomanes thysanostomum Makino (1899) 46].

Other species:

Crepidomanes aphlebioides (H. Christ) I.M. Turner; C. grande (Copel.) Ebihara \& K. Iwats.

\section{POLYPHLEBIUM}

Polyphlebium Copel. (1938b) 55. - Phlebiophyllum Bosch (1861a) 321, non Phlebophyllum Nees in Wallich (1832) 75, 102. - Trichomanes L. sect. Phlebiophyllum (Bosch) Prantl (1875) 52. - Trichomanes L. subg. Polyphlebium (Copel.) Allan (1961) 34. - Type: Polyphlebium venosum (R.Br.) Copel.

Rhizomes long-creeping, frequently branching, filiform, $0.1-0.8 \mathrm{~mm}$ diam., densely covered with light brown hairs, protostele subcollateral, cortex with centrifuge sclerification, roots few and fine. Stipes $0.3-5.5 \mathrm{~cm}$ long, at a distance from the adjacent ones. Blades pinnate to quadripinnate, ovate to narrowly ovate, 30 by $6.5 \mathrm{~cm}$, venation anadromous, sometimes a single row of elongate marginal cells present, false veinlets absent, internal cell walls thin and straight. Sori paratactic, tubular, lips usually dilate, receptacle long-exserted.

Distribution - Temperate regions of the southern hemisphere, and mountain forests of low altitude regions; c. 15 species.

Habitat - Epilithic on wet rocks or epiphytic on tree-fern trunks.

Chromosome base number $-\mathrm{x}=36$.

Note - Corresponding to the Tp clade in Ebihara et al. (submitted). Though constituents of this genus have so far been confused with those of Crepidomanes and Vandenboschia of the present system, their stem and root features well differentiate them from the others.

\section{REPRESENTATIVE SPECIES:}

\section{New combinations:}

Polyphlebium angustatum (Carmich.) Ebihara \& Dubuisson, comb. nov. [based on Trichomanes angustatum Carmich. (1818) 513]; P. borbonicum (Bosch) Ebihara \& Dubuisson, comb. nov. [based on Trichomanes borbonicum Bosch (1861b) 158]; P. capillaceum (L.) Ebihara \& Dubuisson, comb. nov. [based on Trichomanes capillaceum L. (1753) 1099]; P. colensoi (Hook.) Ebihara \& K. Iwats., comb. nov. [based on Trichomanes colensoi Hook. (1854) t. 979]; P. diaphanum (Kunth) Ebihara \& Dubuisson, comb. nov. [based on Trichomanes diaphanum Kunth (1815) 25]; P. endlicherianum (C. Presl) Ebihara \& K. Iwats., comb. nov. [based on Trichomanes endlicherianum C. Presl (1848) 333]; P. exsectum (Kunze) Ebihara \& Dubuisson, comb. nov. [based on Trichomanes exsectum Kunze (1837) 47, t. 29, f. 2]; P. hymenophylloides (Bosch) Ebihara \& Dubuisson, comb. nov. [based on Trichomanes hymenophylloides Bosch (1863) 209]; P. ingae (C. Chr.) Ebihara \& Dubuisson, comb. nov. [based on Trichomanes ingae C. Chr. in C. Chr. \& Skottsb. (1920) 3, f. 2]; P. philippianum (Sturm) Ebihara \& Dubuisson, comb. nov. [based on Trichomanes philippianum Sturm (1858) 188]; P. pyxidi- 
ferum (L.) Ebihara \& Dubuisson, comb. nov. [based on Trichomanes pyxidiferum L. (1753) 1098]; P. vieillardii (Bosch) Ebihara \& K. Iwats., comb. nov. [based on Trichomanes vieillardii Bosch (1861c) 90]; P. werneri (Rosenst.) Ebihara \& K. Iwats., comb. nov. [based on Trichomanes werneri Rosenst. (1908a) 35].

Other species:

Polyphlebium venosum (R.Br.) Copel.

\section{VANDENBOSCHIA}

Vandenboschia Copel. (1938b) 51. - Trichomanes L. subg. Vandenboschia (Copel.) Allan (1961) 34. - Type: Vandenboschia radicans (Sw.) Copel.

Distribution - Throughout the tropics, extending to northern temperate regions; more than 15 species.

Habitat - Hemi-epiphytic on tree trunks or epilithic, occasionally terrestrial.

Chromosome base number $-\mathrm{x}=36$.

Note - Corresponding to the Va clade in Ebihara et al. (submitted). Our molecular analysis shows Copeland's genus Vandenboschia evidently of polyphyletic origin - he included species of at least four different lineages (the Va, Po, PT, NT clades in Ebihara et al., submitted). Vandenboschia, in the present system, refers to V. radicans, type of Vandenboschia, and its relatives. Nakaike (1975) advocated the application of the generic name Lacosteopsis instead of Vandenboschia on the grounds that Vandenboschia was an invalid generic name since Copeland (1938b) included the type of an existing genus (T. scandens) when he established Vandenboschia (T. Nakaike, pers. comm.). This confusion was attributed to the controversial typification of Trichomanes as noted above. Recent nomenclatural conservation of $T$. crispum against $T$. scandens (Greuter et al., 2000) denied the claim that Vandenboschia is an illegitimate name (Morton, 1968; Holttum, 1976).

\section{KEY TO THE SUBGENERA}

1a. Blades bipinnate or more finely divided, hemi-epiphytic or epilithic . . . . . . . $\ldots \ldots \ldots \ldots \ldots \ldots \ldots \ldots \ldots \ldots \ldots \ldots \ldots \ldots \ldots$ 1. Subg. Vandenboschia b. Blades simply pinnate, hemi-epiphytic . . . . . . . . 2. Subg. Lacosteopsis

\section{Subgenus Vandenboschia}

Rhizomes short- or long-creeping, irregularly branching, rather thick, to $1.7 \mathrm{~mm}$ diam., densely covered with brown to bright brown multicellular hairs, protostele reduced, cortex heterogeneous, roots numerous and robust. Stipes 1-16 cm long, clustered or irregularly distanced. Blades bipinnate to 5-pinnatifid, ovate to linear-ovate, 40 by $20 \mathrm{~cm}$, venation anadromous, false veinlets absent, laminae often reduced, regular arrangement of elongate cells observed in some species, internal cell walls various (thin to thick, straight to coarsely pitted). Sori paratactic, tubular to campanulate, lips sometimes dilate, receptacles long-exserted.

Distribution - Throughout the tropics, extending to northern temperate regions; more than 15 species. 
Habitat - Terrestrial in humid places, or epiphytic on tree trunks including tree ferns.

\section{REPRESENTATIVE SPECIES:}

New combinations:

Vandenboschia boschiana (Sturm ex Bosch) Ebihara \& K. Iwats., comb. nov. [based on Trichomanes boschianum Sturm ex Bosch (1861b) 160]; V. collariata (Bosch) Ebihara \& K. Iwats., comb. nov. [based on Trichomanes collariatum Bosch (1859) 368]; V. gigantea (Bory ex Willd.) Ebihara \& Dubuisson, comb. nov. [based on Trichomanes giganteum Bory ex Willd. (1810) 514].

Other species:

Vandenboschia birmanica (Bedd.) Ching; V. cyrtotheca (Hillebr.) Copel.; V. davallioides (Gaudich.) Copel.; V. johnstonensis (F.M. Bailey) Copel.; V. liukiuensis (Y. Yabe) Copel.; V. maxima (Blume) Copel.; V. radicans (Sw.) Copel.; V. speciosa (Willd.) G. Kunkel; V. subclathrata K. Iwats.

\section{Subgenus Lacosteopsis (Prantl) Ebihara \& K. Iwats., comb. nov.}

Based on Trichomanes L. sect. Lacosteopsis Prantl (1875) 53. - Lacosteopsis (Prantl) Nakaike (1975)

21. - Lectotype: Trichomanes luschnatianum C. Presl (= Vandenboschia rupestris Ebihara \& K. Iwats., comb. nov. [based on Hymenophyllum rupestre Raddi (1825) 67, t. 80]) (selected by Christensen (1906) XV, as Trichomanes rupestre (Raddi) Bosch).

Rhizomes suberect, or short- to long-creeping, irregularly branching, wiry, densely covered with brown to bright brown multicellular hairs, or nearly glabrous in climbing parts, protostele reduced, cortex heterogeneous, roots numerous and robust at the terrestrial parts. Stipes $1-16 \mathrm{~cm}$ long, clustered or irregularly distanced. Blades usually pinnate, narrowly elliptic to linear-ovate, 50 by $6 \mathrm{~cm}$, venation anadromous, false veinlets absent, laminae often reduced, regular arrangement of elongated cells observed in some species, internal cell walls various (thin to thick, straight to coarsely pitted). Sori paratactic, tubular to campanulate, lips sometimes dilate, receptacles long-exserted.

Distribution - Both New World and Old World tropics (excl. Africa); at least 2 species.

Habitat - Hemi-epiphytic, climbing on tree trunks.

REPRESENTATIVE SPECIES:

Vandenboschia auriculata (Blume) Copel.; V. rupestris (Raddi) Ebihara \& K. Iwats.

\section{ABRODICTYUM}

Abrodictyum C. Presl (1843) 20, t. VII. - Trichomanes L. sect. Abrodictyum (C. Presl) T. Moore (1857) cx. - Cephalomanes C. Presl subg. Abrodictyum (C. Presl) K. Iwats. (1984) 176. - Habrodictyon C. Presl ex Bosch (1861a) 321. - Type: Abrodictyum cumingii C. Presl.

Distribution - Throughout the tropics; c. 25 species.

Chromosome base number $-\mathrm{x}=33$ (a few doubtful records $\mathrm{x}=36$ ).

Note - Corresponding to the Pa clade in Ebihara et al. (submitted). Circumscription of this genus is very similar to Morton's subgenus Pachychaetum except for the inclusion of Abrodictyum and the exclusion of Davalliopsis. Dissected fronds consisting primarily of the axis (lacking laminar cells) is a unique character observed in some species of this genus, but according to molecular results such fronds evolved several times in parallel within this genus. 


\section{KEY TO THE SUBGENERA}

1a. Distribution in Asia/Pacific ......................... 2

b. Distribution in Africa/America . . . . . . . . . . . 2. Subg. Pachychaetum

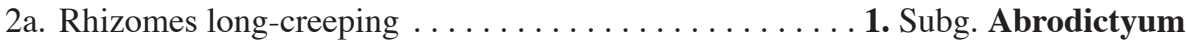

b. Rhizomes erect or short-creeping $\ldots \ldots \ldots \ldots \ldots \ldots \ldots \ldots \ldots \ldots \ldots \ldots \ldots$

3a. Lamina texture membranous . . . . . . . . . . . . Subg. Abrodictyum

b. Lamina texture not membranous . . . . . . . . . . 2. Subg. Pachychaetum

\section{Subgenus Abrodictyum}

Trichomanes L. sect. Leptomanes Prantl (1875) 52, nom illeg.; one of the five original species, Trichomanes smithii Hook. (= Abrodictyum cumingii C. Presl), is also the type of Trichomanes L. sect. Abrodictyum (C. Presl) T. Moore (1857).

Rhizomes suberect, or short- to long-creeping, irregularly branching, rather thick, to $1.7 \mathrm{~mm}$ diam., densely covered with brown to bright brown multicellular hairs, protostele massive, cortex homogeneous, roots numerous and robust. Stipes $1-16 \mathrm{~cm}$ long, clustered or irregularly distanced. Blades bipinnate to 5-pinnatifid, narrowly ovate to linear-ovate, 40 by $6 \mathrm{~cm}$, venation anadromous, false veinlets absent, laminae often reduced, regular arrangement of elongate cells observed in some species, internal cell walls various (thin to thick, straight to coarsely pitted). Sori paratactic, tubular to campanulate, lips sometimes dilate, receptacles long-exserted.

Distribution - Paleotropics (Asia to the Pacific); c. 15 species.

Habitat - Terrestrial in humid places, or epiphytic on tree trunks including tree ferns.

Note - Corresponding to the Ab clade in Ebihara et al. (submitted).

REPRESENTATIVE SPECIES:

New combinations:

Abrodictyum asae-grayi (Bosch) Ebihara \& K. Iwats., comb. nov. [based on Trichomanes asaegrayi Bosch (1861b) 180]; A. brassii (Croxall) Ebihara \& K. Iwats., comb. nov. [based on Macroglena brassii Croxall (1975) 543]; A. caudatum (Brack.) Ebihara \& K. Iwats., comb. nov. [based on Trichomanes caudatum Brack. (1854) 256, pl. 36, f. 5]; A. clathratum (Tagawa) Ebihara \& K. Iwats., comb. nov. [based on Trichomanes clathratum Tagawa (1939) 164]; A. flavofuscum (Bosch) Ebihara \& K. Iwats., comb. nov. [based on Trichomanes flavofuscum Bosch (1861c) 88]; A. idoneum (C.V. Morton) Ebihara \& K. Iwats., comb. nov. [based on Trichomanes idoneum C.V. Morton (1973) 272] (Old World plants formerly called 'T. gemmatum' are this species, because T. gemmatum J. Sm. ex Baker was originally applied to a Neotropical plant (see Morton, 1973)); A. pluma (Hook.) Ebihara \& K. Iwats., comb. nov. [based on Trichomanes pluma Hook. (1854) t. 997] (Asian plants formerly identified as 'T. meifolium' are this species); A. schlechteri (Brause) Ebihara \& K. Iwats., comb. nov. [based on Trichomanes schlechteri Brause (1912) 10]; A. strictum (Menzies ex Hook. \& Grev.) Ebihara \& K. Iwats., comb. nov. [based on Trichomanes strictum Menzies ex Hook. \& Grev. (1831) t. 122].

Other species:

Abrodictyum boninense Tagawa \& K. Iwats.; A. cumingii C. Presl.

2. Subgenus Pachychaetum (C. Presl) Ebihara \& K. Iwats., comb. nov.

Based on Trichomanes L. subg. Pachychaetum C. Presl (1843) 16. - Cephalomanes C. Presl subg. Pachychaetum (C. Presl) K. Iwats. (1984) 177. - Lectotype: Trichomanes rigidum Sw. (= Abrodictyum rigidum (Sw.) Ebihara \& Dubuisson, comb. nov. [based on Trichomanes rigidum Sw. (1788) 137]) (selected by Christensen (1906) XV). 
Trichomanes L. subg. Macroglena C. Presl (1848) 333. - Macroglena (C. Presl) Copel. (1938a) 49. - Cephalomanes C. Presl subg. Macroglena (C. Presl) K. Iwats. (1984) 176. - Lectotype: Trichomanes meifolium Bory ex Willd. (1810) 509 (= Abrodictyum meifolium (Bory ex Willd.) Ebihara \& K. Iwats., comb. nov.) (selected by Christensen (1906) XV).

Trichomanes L. sect. Selenodesmium Prantl (1875) 53. - Selenodesmium (Prantl) Copel. (1938b) 80. - Lectotype: Trichomanes rigidum Sw. (= Abrodictyum rigidum (Sw.) Ebihara \& Dubuisson) (selected by Christensen (1906) XV).

Rhizomes erect or short-creeping, quite thick, about $2 \mathrm{~mm}$ diam., covered with brown to blackish hairs, protostele massive, cortex homogeneous, roots numerous and robust. Stipes $7-15 \mathrm{~cm}$ long, clustered or more or less distanced. Blades bipinnate to quadripinnatifid, linear-ovate to subdeltate, 30 by $18 \mathrm{~cm}$, venation anadromous, false veinlets absent, laminae reduced in some species, internal cell walls thick and coarsely pitted. Sori paratactic, tubular, lips truncate, receptacles long-exserted.

Distribution - Throughout the tropics; more than 10 species.

Habitat - Usually terrestrial in humid places, occasionally low-epiphytic.

Note - Corresponding to the Pa subclade in Ebihara et al. (submitted).

REPRESENTATIVE SPECIES:

New combinations:

Abrodictyum cellulosum (Klotzsch) Ebihara \& Dubuisson, comb. nov. [based on Trichomanes cellulosum Klotzsch (1844) 531]; A. cupressoides (Desv.) Ebihara \& Dubuisson, comb. nov. [based on Trichomanes cupressoides Desv. (1827) 330]; A. dentatum (Bosch) Ebihara \& K. Iwats., comb. nov. [based on Trichomanes dentatum Bosch (1861b) 182]; A. elongatum (A. Cunn.) Ebihara \& K. Iwats., comb. nov. [based on Trichomanes elongatum A. Cunn. (1836) 368]; A. kalimantanense (K. Iwats. \& M. Kato) Ebihara \& K. Iwats., comb. nov. [based on Macroglena kalimantanensis K. Iwats. \& M. Kato (1980) 31, f. 1]; A. laetum (Bosch) Ebihara \& K. Iwats., comb. nov. [based on Trichomanes laetum Bosch (1861c) 90]; A. obscurum (Blume) Ebihara \& K. Iwats., comb. nov. [based on Trichomanes obscurum Blume (1828) 227] (there are two varieties under this species: var. obscurum and var. siamense (H. Christ) K. Iwats., comb. nov. [based on Trichomanes siamense H. Christ (1901) 103]); A. setaceum (Bosch) Ebihara \& K. Iwats., comb. nov. [based on Trichomanes setaceum Bosch (1861b) 176]; A. sprucei (Baker) Ebihara \& Dubuisson, comb. nov. [based on Trichomanes sprucei Baker in Hook. \& Baker (1867) 87]; A. tamarisciforme (Jacq.) Ebihara \& Dubuisson, comb. nov. [based on Trichomanes tamarisciforme Jacq. (1789) 285, t. 21, f. 2].

Other species:

Abrodictyum meifolium (Bory ex Willd.) Ebihara \& K. Iwats.; A. rigidum (Sw.) Ebihara \& Dubuisson.

\section{TRICHOMANES}

Trichomanes L. (1753) 1097. - Type: Trichomanes crispum L., type. cons., ICBN 2000 (vs Trichomanes scandens L.).

Distribution - The Neotropics, and at least one species in continental Africa; more than 60 species. Unpublished molecular data suggest inclusion of a few other Paleotropical taxa (from Madagascar and the Indian Ocean), but further phylogenetic investigations are needed.

Chromosome base number $-\mathrm{x}=32$ (a few doubtful records $\mathrm{x}=36$ ).

Note - Corresponding to the NT clade in Ebihara et al. (submitted). Constituents of this genus include Morton's subgenus Achomanes and sect. Davalliopsis (placed under subg. Pachychaetum) along with T. scandens (placed under subg. Trichomanes). In 
contrast to its long-term acceptance in New World floras, Morton's subgenus Achomanes shared few morphological characters other than geographical distribution and chromosome base number. Recent inclusion of Davalliopsis in this group (Dubuisson et al., 2003a; Ebihara et al, submitted) made it even more difficult to define morphologically. We therefore recognize four subgenera under this genus, which are easily definable. Monophyly of subgenus Davalliopsis and subg. Lacostea is clearly shown by our molecular analysis (as the Da subclade), but they are very different in most morphological features.

\section{KEY TO THE SUBGENERA}

1a. Lamina texture membranous, one cell thick ................ 2

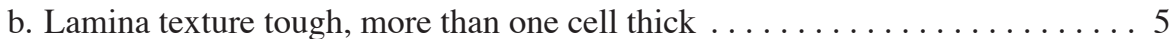

2a. Rhizomes short-creeping or erect ........... 1. Subg. Trichomanes

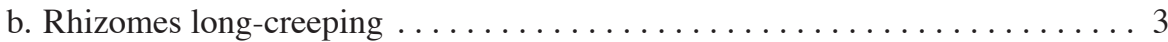

3a. Blades tripinnate or more finely divided ........ 1. Subg. Trichomanes

b. Blades once-pinnate. ....................... 4

4a. Margins of blades hairy . . . . . . . . . . . . . Subg. Trichomanes

b. Margins of blades glabrous . . . . . . . . . . . . . . 4. Subg. Lacostea

5a. Venation catadromous in sterile blades . . . . . . . . . . . 2. Subg. Feea

b. Venation anadromous in sterile blades . . . . . . . . 3. Subg. Davalliopsis

\section{Subgenus Trichomanes}

Trichomanes L. subg. Eutrichomanes C. Presl (1843) 16. - Trichomanes L. sect. Eutrichomanes (C. Presl) T. Moore (1857) cx. - Lectotype: Trichomanes scandens L. (selected by Morton (1968) 180; his idea that this and several subsequent synonyms include the type species of Trichomanes led him to designate $T$. scandens as lectotypes for these taxa. Although T. crispum has recently been fixed to the type of the genus as noted above, Morton's lectotypifications do not need to be changed since they were validly published).

Trichomanes L. sect. Pinnata C. Presl (1843) 16. - Lectotype: Trichomanes scandens L. (selected by Morton (1968) 180).

Ragatelus C. Presl (1843) 16. - Trichomanes L. sect. Ragatelus (C. Presl) C. Chr. (1906) XIV. - Type: Ragatelus crinitus (Sw.) C. Presl (= Trichomanes crinitum Sw.).

Homoeotes C. Presl (1848) 331. - Ptilophyllum Bosch sect. Homoeotes (C. Presl) Prantl (1875) 48. - Trichomanes L. sect. Homoeotes (C. Presl) C. Chr. (1906) XIV. - Type: Homoeotes heterophylla (Willd.) C. Presl (= Trichomanes humboldtii (Bosch) Lellinger).

Trichomanes L. subg. Pseudoachomanes C. Presl (1849) 16. - Trichomanes L. sect. Pseudoachomanes (C. Presl) C. Chr. (1906) XIV. - Lectotype: Trichomanes sinuosum Rich. ex Willd. (= Trichomanes polypodioides L.) (selected by Christensen (1906) XIV, as T. polypodioides L.).

Odontomanes C. Presl (1849) 20. - Trichomanes L. sect. Odontomanes (C. Presl) C. Chr. (1906) XIV. - Type: Odontomanes hostmannianum (Klotzsch) C. Presl (= Trichomanes hostmannianum (Klotzsch) Kunze).

Trichomanes L. sect. Neurophyllum T. Moore (1857) cx, based on Neurophyllum C. Presl (1843) 18, t. IV, f. C, nom. illeg., non Torr. \& A. Gray. (1840) 612. - Neuromanes Trevis. ex Bosch (1859) 347. - Type: Neurophyllum pinnatum (Hedw.) C. Presl (= Trichomanes pinnatum Hedw.) (selected by Christensen (1906) XIV).

Ptilophyllum Bosch subsect. Sinuosa Prantl (1875) 47. - Lectotype: Ptilophyllum sinuosum (Rich. ex Willd.) Prantl (= Trichomanes polypodioides L.) (selected by Morton (1968) 198). 
Ptilophyllum Bosch sect. Acarpacrium Prantl (1875) 48. - Trichomanes L. sect. Acarpacrium (Prantl) C. Chr. (1906) XIV. - Lectotype: Ptilophyllum ptilodes (Bosch) Prantl (= Trichomanes alatum Sw.) (selected by Christensen (1906) XIV, as T. alatum Sw.).

Ptilophyllum Bosch sect. Trigonophyllum Prantl (1875) 48. - Trichomanes L. sect. Trigonophyllum (Prantl) C. Chr. (1906) XIV. - Lectotype: Ptilophyllum bancroftii (Hook. \& Grev.) Prantl (= Trichomanes arbuscula Desv.) (selected by Christensen (1906) XIV, as T. arbuscula Desv.).

Ptilophyllum Bosch subsect. Lamellata Prantl (1875) 48. - Trichomanes L. subsect. Lamellata (Prantl) C.V. Morton (1968) 194. - Type: Ptilophyllum martiusii (C. Presl) Prantl (= Trichomanes martiusii C. Presl).

Trichomanes L. sect. Maiora Prantl (1875) 52. - Crepidomanes (C. Presl) C. Presl subg. Maiora K. Iwats. (1984) 174. - Lectotype: Trichomanes scandens L. (selected by Morton (1968) 181).

Trichomanes L. subg. Holophlebium H. Christ (1897) 27. - Lectotype: Trichomanes scandens L. (selected by Morton (1968) 181).

Mortoniopteris Pic.Serm. (1977a) 243. - Trichomanes L. sect. Mortoniopteris (Pic. Serm.) C. Sánchez (2000) 39. - Type: Mortoniopteris scandens (L.) Pic.Serm. (= Trichomanes scandens L.).

Pteromanes Pic. Serm. (1977a) 244. - Type: Pteromanes martiusii (C. Presl) Pic.Serm. (= Trichomanes martiusii C. Presl).

Trichomanes L. subg. Achomanes C. Presl (1843) 15, nom. illeg.; originally including Trichomanes crispum L., the type of the genus.

Ptilophyllum Bosch (1861a) 321, nom. illeg., non Morris in C.W. Grant (1840) 327, nec (Nutt.) Rchb. (1841) 169; originally including Trichomanes crinitum Sw., the type of Ragatelus C. Presl (1843).

Ptilophyllum Bosch subsect. Crispa Prantl (1875) 47, nom. illeg.; originally including Trichomanes crispum $\mathrm{L}$. , type of the genus.

Rhizomes erect, short- or long-creeping, usually fine to quite thick, 1-5 mm diam., occasionally filiform, sparsely or sometimes densely covered with brown to blackish hairs, protostele massive, cortex homogeneous, or occasionally heterogeneous (in T. scandens), roots numerous and robust. Stipes to $30 \mathrm{~cm}$ long, usually close to each other, sometimes more or less distant. Blades usually pinnate with symmetric pinnae, occasionally simple or more finely pinnate, ovate to linear-ovate, 70 by $25 \mathrm{~cm}$, venation usually catadromous, or anadromous (in T. scandens and T. anadromum), sometimes pubescent with unicellular or multicellular hairs, false veinlets present in some species (perpendicular to true veins), internal cell walls slightly thick and wavy, margins sometimes hairy with simple or stellate hairs. Sori paratactic, or epitactic (in T. scandens), often immersed in the laminae, rarely pedicellate (in T. pinnatum), tubular, lips sometimes dilate, receptacles usually long-exserted.

Distribution - Same as that of the genus; more than 30 species.

Habitat - Usually terrestrial in humid places, sometimes epiphytic on tree trunks, exceptionally as canopy epiphytes (T. pilosum). Some taxa such as T. crispum appear ecologically opportunistic.

Note - Corresponding to the Ac subclade in Ebihara et al. (submitted).

\section{REPRESENTATIVE SPECIES:}

Trichomanes accedens $\mathrm{C}$. Presl; T. alatum Sw.; T. anadromum Rosenst.; T. anomalum Maxon \& C.V. Morton; T. arbuscula Desv.; T. bicorne Hook.; T. crenatum Bosch; T. crinitum Sw.; T. crispum L.; T. cristatum Kaulf.; T. dactylites Sodiro; T. delicatum Bosch; T. egleri P.G. Windisch; T. fimbriatum Backh. ex T. Moore; T. galeottii E. Fourn.; T. guidoi P. G. Windisch; T. holopterum Kunze; T. hostmannianum (Klotzsch) Kunze; T. humboldtii (Bosch) Lellinger; T. kalbreyeri Baker; T. lucens Sw.; T. ludovicinum Rosenst.; T. macilentum Bosch; T. martiusii C. Presl; T. micayense Hieron.; T. pellucens Kunze; T. pilosum Raddi; T. pinnatum Hedw.; T. plumosum Kunze; T. polypodioides L.; 
T. robustum E. Fourn.; T. roraimense Jenman; T. scandens L.; T. spruceanum Hook.; T. steyermarkii P.G. Windisch \& A.R. Sm.; T. trigonum Desv.; T. vandenboschii P.G. Windisch; T. vaupesense Lellinger; T. vittaria DC. ex Poir.

\section{Subgenus Feea (Bory) Hook.}

Trichomanes L. subg. Feea (Bory) Hook. (1844) 114. - Feea Bory (1824) 446. - Ptilophyllum Bosch sect. Feea (Bory) Prantl (1875) 48. — Trichomanes L. sect. Feea (Bory) H. Christ (1897) 29. - Lectotype: Feea polypodina Bory (= Trichomanes osmundoides Poir.) (selected by Smith (1875) 349, as Trichomanes spicatum Hedw. ex Hook.).

Hymenostachis Bory (1824) 588. — Trichomanes L. subg. Hymenostachis (Bory) Hook. (1844) 114. - Trichomanes L. subsect. Hymenostachis (Bory) C.V. Morton (1968) 197. - Type: Trichomanes elegans Rudge (= Trichomanes diversifrons (Bory) Mett.).

Maschalosorus Bosch (1861a) 320. - Trichomanes L. sect. Maschalosorus (Bosch) C. Chr. (1906) XIV. - Type: Maschalosorus mougeotii (Bosch) Bosch (= Trichomanes mougeotii Bosch).

Rhizomes suberect or ascending (leading to a dwarf tree-fern habit), quite thick, 1.5-8 mm diam., apex covered with brown to blackish hairs, basal parts covered by roots, protostele massive, cortex homogeneous, root numerous and robust. Stipes to $8 \mathrm{~cm}$ long, usually close to each other. Fronds dimorphic (except in T. mougeotii), simple or without lamina in the fertile fronds, once-pinnate in the sterile fronds, 30 by $8 \mathrm{~cm}$, venation catadromous (in sterile frond), false veinlets absent, laminae usually more than one cell thick in fertile fronds, internal cell walls thick and coarsely pitted. Sori paratactic or pantotactic, immersed in the lamina, tubular, lips truncate or slightly dilate, receptacles long-exserted.

Distribution - The Neotropics; more than 5 species.

Habitat - Terrestrial or epilithic.

Note - Corresponding to the Fe subclade in Ebihara et al. (submitted), and also to Copeland's (1938b) genus, Feea.

REPRESENTATIVE SPECIES:

Trichomanes botryoides Kaulf.; T. diversifrons (Bory) Mett.; T. mougeotii Bosch; T. osmundoides Poir.; T. trollii Bergdolt.

\section{Subgenus Davalliopsis (Bosch) Ebihara \& K. Iwats., comb. nov.}

Based on Davalliopsis Bosch (1861a) 323. - Trichomanes L. sect. Davalliopsis (Bosch) Prantl (1875)

53. - Cephalomanes C. Presl subg. Davalliopsis (Bosch) K. Iwats. (1984) 177. - Lectotype:

Trichomanes prieurii Kunze (= Trichomanes elegans Rich.) (selected by Prantl (1875) 53).

Rhizomes erect or ascending, quite thick, 4-15 mm diam., densely covered with dark hairs, protostele massive, cortex homogeneous, roots numerous and robust. Stipes to $50 \mathrm{~cm}$ long, close to each other. Blades tri- to quadripinnatifid, deltate, 60 by $36 \mathrm{~cm}$, venation anadromous, false veinlets absent, laminae usually more than one cell thick, internal cell walls thick and coarsely pitted. Sori paratactic, tubular, lips truncate or slightly dilate, receptacles long-exserted.

Distribution - The Neotropics; at least 1 species.

Habitat - Terrestrial.

Note - Corresponding to a part of the Da subclade in Ebihara et al. (submitted).

REPRESENTATIVE SPECIES:

Trichomanes elegans Rich. 
4. Subgenus Lacostea (Bosch) C. Chr.

Trichomanes L. subg. Lacostea (Bosch) C. Chr. (1906) 634. - Lacostea Bosch (1861a) 320. - Trichomanes L. sect. Lacostea (Bosch) H. Christ (1897) 29. - Lectotype: Lacostea brachypus (Kunze) Prantl (= Trichomanes pedicellatum Desv.) (selected by Christensen (1906) XIV, as 'T. pedicellatum Desv.').

Rhizomes long-creeping, frequently branching, rather thick, $0.5-1.2 \mathrm{~mm}$ diam., sparsely to densely covered with brown hairs, protostele massive, cortex heterogeneous, roots numerous and robust terrestrially, rootless at the climbing part. Stipes very short and unclear, subdistant to remote. Blades once-pinnate with symmetric pinnae, occasionally finer (up to tripinnatifid), elliptic, 30 by $9 \mathrm{~cm}$, venation anadromous, false veinlets absent, internal cell walls slightly thick and wavy. Sori paratactic, tubular, lips truncate or sometimes dilate, receptacles long-exserted.

Distribution - The Neotropics; more than 4 species.

Habitat - Lianas on tree trunks.

Note - Corresponding to a part of the Da subclade in Ebihara et al. (submitted).

REPRESENTATIVE SPECIES:

Trichomanes ankersii C. Parker ex Hook. \& Grev.; T. pedicellatum Desv.; T. tanaicum Hook.; T. tuerckheimii H. Christ.

\section{CEPHALOMANES}

Cephalomanes C. Presl (1843) 17, t. V. - Lacostea Bosch sect. Cephalomanes (C. Presl) Prantl (1875) 50. - Trichomanes L. subg. Cephalomanes (C. Presl) C. Chr. (1906) XIV. - Trichomanes L. sect. Cephalomanes (C. Presl) C.V. Morton (1968) 189. - Type: Cephalomanes atrovirens C. Presl.

Rhizomes erect to short-creeping, thick, usually densely covered with old stipes, protostele massive, cortex homogeneous, roots numerous and robust. Stipes $2-15 \mathrm{~cm}$ long, close to each other. Blades once-pinnate with asymmetric pinnae, narrowly elliptic, $10-20$ by $3-4(-5) \mathrm{cm}$, venation anadromous, false veinlets absent, internal cell walls thick and coarsely pitted. Sori paratactic, campanulate, lips sometimes dilate, receptacles long-exserted.

Distribution - The Paleotropics (Asia to the Pacific); c. 4 species.

Habitat - Terrestrial or epilithic along streams.

Chromosome base number $-\mathrm{x}=32$.

Note - Corresponding to the Ce clade in Ebihara et al. (submitted).

REPRESENTATIVE SPECIES:

Cephalomanes atrovirens C. Presl; C. crassum (Copel.) M.G. Price; C. javanicum (Blume) C. Presl. The Malagasian Trichomanes madagascariense (Bosch) Moore has traditionally been placed in this group, but unpublished molecular data suggest its exclusion. Further investigations are needed.

\section{CALLISTOPTERIS}

Callistopteris Copel. (1938b) 64. - Trichomanes L. sect. Callistopteris (Copel.) C.V. Morton (1968) 190. - Cephalomanes C. Presl subg. Callistopteris (Copel.) K. Iwats. (1984) 176. - Type: Callistopteris apiifolia (C. Presl) Copel.

Rhizomes erect to short-creeping, quite thick, hairy at apices, protostele massive, cortex homogeneous, roots numerous and robust. Stipes $5-25 \mathrm{~cm}$ long, close to each other, 
densely hairy with long bristle-like light reddish hairs. Blades tripinnate to quadripinnate, elliptic to narrowly ovate, 40 by $16 \mathrm{~cm}$, venation anadromous, false veinlets absent, internal cell walls thin and straight. Sori paratactic, campanulate, lips truncate, or sometimes bilabiate, receptacles long-exserted.

Distribution - The Paleotropics (Asia to the Pacific); c. 5 species.

Habitat - Usually epilithic on moist rocks, occasionally epiphytic on tree trunks.

Chromosome base number $-\mathrm{x}=36$.

Note - Corresponding to the Ca clade in Ebihara et al. (submitted).

REPRESENTATIVE SPECIES:

\section{New combination:}

Callistopteris superba (Backh. ex T. Moore) Ebihara \& K. Iwats., comb. nov. [based on Trichomanes superbum Backh. ex T. Moore (1862) 44].

\section{Other species:}

Callistopteris apiifolia (C. Presl) Copel.; C. baldwinii (D.C. Eaton) Copel.; C. baueriana (Endl.) Copel.; C. polyantha (Hook.) Copel.

\section{ACKNOWLEDGEMENTS}

We are grateful to Ms. E. Wood (Harvard University Herbaria) for checking and correcting the English manuscript; to Dr. P.D. Bostock (Queensland Herbarium) for checking the Latin diagnosis; to the directors and curators of herbaria (BM, K, L and P) for supporting our investigation of their specimens; to Dr. N. Murakami and Dr. H. Nishida for providing some literature; to Dr. Leon Perrie (Museum of New Zealand Te Papa Tongarewa) for giving advices about the replacement for Cardiomanes reniforme and to the anonymous reviewer for valuable comments on the manuscript. This study was partly supported by the JSPS Grant for Oversea Research No. 12575012 (for K.I.) and by JSPS Fellows (for A.E.).

\section{REFERENCES}

Alderw. - See Van Alderwerelt van Rosenburgh, C.R.W.K.

Allan, H.H.B. 1961. Flora of New Zealand, vol. 1. Government Printer, Wellington.

Baker, J.G. 1866. Some new species of Hymenophyllaceae. J. Linn. Soc., Bot. 9: 335-341.

Baksh-Comeau, Y.S. 2000. Checklist of the pteridophytes of Trinidad \& Tobago. Fern Gaz. 16: $11-122$.

Bentham, G. 1862. Leguminosae. Dalbergiae. In: C.F.P. Martius \& A.G. Eichler (eds.), Flora Brasiliensis. Vol. 15, 1: 1-349. Fleischer, Leipzig.

Blume, C.L. 1828. Enumeratio Plantarum Javae. Van Leeuwen, Lugduni Batavorum.

Bonaparte, R. 1920. Les ptéridophytes de Madagascar, première partie. Notes Pteridol. 9: 1-44.

Bory, J.B.G.G.M. 1824. Dictionnaire classique d'histoire naturelle, vol. 6. Publisher unknown, Paris.

Bosch - See Van den Bosch, R.B.

Bostock, P.D. \& T.M. Spokes. 1998. Hymenophyllaceae. In: P. M. McCarthy (ed.), Flora of Australia. Vol. 48: 116-148. Australian Biological Resources Study, Canberra.

Brackenridge, W.D. 1854. Botany - Cryptogamia - Filices. U.S. Expl. Exped. 16: 250-268.

Brause, G. 1912. Neue Farne Papuasiens. Bot. Jahrb. Syst. 49: 6-59.

Carmichael, D. 1818. Some account of the island of Tristan da Cunha and of its natural production. Trans. Linn. Soc. London 12: 483-513.

Ching, R.C. 1959. Pteridophyta. Flora Reipublicae Popularis Sinicae. Science Press, Beijing.

Christ, K.H.H. 1897. Farnkräuter der Erde. Fischer, Jena.

Christ, K.H.H. 1901. Pteridophyta. In: J. Schmidt (ed.), Flora of Koh Chang. Bot. Tidsskr. 24: 102-114. 
Christ, K.H.H. 1908a. Spicilegium filicum Philippinensium novarum aut imperfecte congnitarum, II. Philipp. J. Sci., Bot. 3: 269-276.

Christ, K.H.H. 1908b. Filices - Novitates florae Africanae. Bull. Soc. Bot. France 55: Mém. 8b, 105-109.

Christ, K.H.H. 1909a. Diagnoses plantarum Africae. Plantes nouvelles de l'Afrique tropicale française décrites d'après les collections de M. Auguste Chevalier, Filices. J. Bot. (Morot) 22: 19-24.

Christ, K.H.H. 1909b. Pteridophyta. In: E. de Wildeman (ed.), Etudes de systématique et de géographie botaniques sur la flore du Bas- et du Moyen-Congo. Ann. Mus. Congo Belge, Bot. 5, 3: 23-41.

Christensen, C. 1905-1906. Index Filicum. Hagerup, Copenhagen.

Christensen, C. 1920. New species of Hymenophyllaceae from Madagascar. Notes Pteridol. 12: $1-20$.

Christensen, C. 1934. Index Filicum, Supplementum Tertium pro Annis 1917-1933. Hagerup, Copenhagen.

Christensen, C. \& C. Skottsberg. 1920. The pteridophyta of the Juan Fernandez Islands. Nat. Hist. Juan Fernandez (Botany) 2: 1-46.

Copeland, E.B. 1911. Papuan ferns collected by the reverend Copland King. Philipp. J. Sci., Bot. 6: $65-92$.

Copeland, E.B. 1933. Trichomanes. Philipp. J. Sci. 51: 119-280.

Copeland, E.B. 1937. Hymenophyllum. Philipp. J. Sci. 64: 1-188.

Copeland, E.B. 1938a. Ferns of southeastern Polynesia. Occas. Pap. Bernice P. Bishop Mus. 14: $45-101$.

Copeland, E.B. 1938b. Genera Hymenophyllacearum. Philipp. J. Sci. 67: 1-110.

Copeland, E.B. 1941. Notes on Hymenophyllaceae. Philipp. J. Sci. 73: 457-469.

Copeland, E.B. 1947. Genera Filicum. Waltham, Massachusetts.

Copeland, E.B. 1958. Fern Flora of the Philippines I. Institute of Science and Technology, Manila.

Croxall, J.P. 1975. The Hymenophyllaceae of Queensland. Austral. J. Bot. 23: 509-547.

Cunningham, A. 1836. Florae insularum Novae Zelandiae precursor: or a specimen of the botany of the islands of New Zealand. Companion Bot. Mag. 2: 358-378.

DC. - see De Candolle, A.

De Candolle, A. 1828. Prodromus Systematis Naturalis Regni Vegetabilis. Vol. 3. Sumptibus Sociorum Treuttel \& Wurtz, Paris.

De la Sota, E.R. 1977. Flora de la Provincia de Jujuy, Republica Argentina. Instituto Nacional de Tecnologia Agropecuaria (INTA), Buenos Aires.

De Vol, C.E. 1975. Flora of Taiwan, Vol. 1. Epoch Publishing, Taipei.

Desvaux, N.A. 1827. Prodrome. De la famille des fougères. Mém. Soc. Linn. Paris 6: 171-337.

Diem, J. \& J.S. de Lichtenstein. 1959. Las Himenofilaceas del area Argentino-Chilena del Sud. Darwiniana 11: 611-760.

Domin, K. 1913. Pteridophyta - Prodromus einer Farnflora Queenslands. Biblioth. Bot. 20, 85: $1-238$.

Du Puy, D.J. \& A.E. Orchard. 1993. Hymenophyllaceae. In: A.S. George et al. (eds.), Flora of Australia. Vol. 50 - Oceanic Islands 2: 538-540. Australian Biological Resources Study, Canberra.

Dubuisson, J.-Y. 1997. rbcL sequences: A promising tool for the molecular systematics of the fern genus Trichomanes (Hymenophyllaceae). Molec. Phylogenet. Evol. 8: 128-137.

Dubuisson, J.-Y., S. Hennequin, E.J.P. Douzery, R.B. Cranfill, A.R. Smith \& K.M. Pryer. 2003a. rbcL phylogeny of the fern genus Trichomanes (Hymenophyllaceae) with special reference to Neotropical taxa. Int. J. Pl. Sci. 164: 753-761.

Dubuisson, J.-Y., S. Hennequin, S. Rakotondrainibe \& H. Schneider. 2003b. Ecological diversity and adaptive tendencies in the tropical fern Trichomanes L. (Hymenophyllaceae) with special reference to climbing and epiphytic habits. Bot. J. Linn. Soc. 142: 41-63.

Dumortier, B.C.J. 1835. Recueil d'observations sur les Jungermanniacées. Publisher unknown, Tournay.

Ebihara, A., S. Hennequin, K. Iwatsuki, P.D. Bostock, S. Matsumoto, R. Jaman, J.-Y. Dubuisson \& M. Ito. 2004. Polyphyletic origin of Microtrichomanes (Prantl) Copel. (Hymenophyllaceae), with a revision of the species assigned to the genus. Taxon 53: 935-948. 
Ebihara, A., K. Iwatsuki, S. Kurita \& M. Ito. 2002. Systematic position of Hymenophyllum rolandiprincipis Rosenst. or a monotypic genus Rosenstockia Copel. (Hymenophyllaceae) endemic to New Caledonia. Acta Phytotax. Geobot. 53: 35-49.

Forster, J.G.A. 1786. Florulae Insularum Australium Prodromus. Dieterich, Göttingen.

Grant, C.W. 1840. Memoir to illustrate a geological map of Cutsch. Trans. Geol. Soc. (London), II, 2, 5: 289-330.

Green, P.S. 1994. Hymenophyllaceae. In: A.J.G. Willson (ed.), Flora of Australia. Vol. 49 - Oceanic Islands 1: 557-561. Australian Biological Resources Study, Canberra.

Greuter, W., J. McNeill, F.R. Barrie, H.M. Burdet, V. Demoulin, T.S. Filgueiras, D.H. Nicolson, P.C. Silva, J.E. Skog, P. Trehane, N.J. Turland \& D.L. Hawksworth. 2000. International Code of Botanical Nomenclature (St Louis Code) adopted by the Sixteenth International Botanical Congress, St Louis, Missouri, July-August 1999. Koeltz Scientific, Königstein.

Hennequin, S., A. Ebihara, M. Ito, K. Iwatsuki \& J.-Y. Dubuisson. 2003. Molecular systematics of the fern genus Hymenophyllum s.l. (Hymenophyllaceae) based on chloroplastic coding and noncoding regions. Molec. Phylogenet. Evol. 27: 283-301.

Hennequin, S., A. Ebihara, M. Ito, K. Iwatsuki \& J.-Y. Dubuisson. In press. New insights into the phylogeny of the genus Hymenophyllum s.l. (Hymenophyllaceae): revealing the polyphyly of Mecodium. Syst. Bot.

Herzog, T.C.J. 1926. Bryophyten der weiteren Indomalaya. Hedwigia 66: 337-358.

Holttum, R.E. 1976. Proposal for the conservation of the generic name Trichomanes Linn. against Vandenboschia Copel. Taxon 25: 203-204.

Hooker, W.J. 1844-1846. Species Filicum, vol. 1. Pamplin, London.

Hooker, W.J. 1854. Icones Plantarum; or Figures, with brief descriptive characters and remarks of new or rare plants, vol. X. Longman, London.

Hooker, W.J. \& J.G. Baker. 1867. Synopsis Filicum; or A synopsis of all known ferns. Hardwicke, London.

Hooker, W.J. \& R.K. Greville. 1831. Icones Filicum, vol. 2. Treuttel, London.

Iwatsuki, K. 1975. Studies in the systematics of filmy ferns I. A note on the identity of Microtrichomanes. Fern Gaz. 11: 115-124.

Iwatsuki, K. 1977a. Studies in the systematics of filmy ferns II. A note on Meringium and the taxa allied this. Gard. Bull. Singapore 30: 63-74.

Iwatsuki, K. 1977b. Studies in the systematics of filmy ferns III. An observation on the involucres. Bot. Mag. (Tokyo) 90: 259-267.

Iwatsuki, K. 1978. Studies in the systematics of filmy ferns IV. Notes on the species with false veinlets. Mem. Fac. Sci. Kyoto Univ., Ser. Biol. 7: 31-43.

Iwatsuki, K. 1981. Studies in the systematics of filmy ferns V. A note on the identity of Macroglena. Hikobia, Suppl. 1: 59-66.

Iwatsuki, K. 1982. Studies in the systematics of filmy ferns VI. The genus Sphaerocionium in Asia and Oceania. J. Fac. Sci. Univ. Tokyo, Sect. 3, Bot. 13: 203-215.

Iwatsuki, K. 1984. Studies in the systematics of filmy ferns VII. A scheme of classification based on the Asiatic species. Acta Phytotax. Geobot. 35: 165-179.

Iwatsuki, K. 1985. The Hymenophyllaceae of Asia, excluding Malesia. J. Fac. Sci. Univ. Tokyo, Sect. 3, Bot. 13: 501-551.

Iwatsuki, K. 1990. Hymenophyllaceae. In: K. Kubitzki (ed.), The families and genera of vascular plants. Vol. I, Pteridophytes and Gymnosperms: 157-163. Springer Verlag, Berlin.

Iwatsuki, K. 1995. Hymenophyllaceae. In: K. Iwatsuki, T. Yamazaki, D.E. Boufford \& H. Ohba (eds.), Flora of Japan. Vol. 1: 41-53. Kodansha, Tokyo.

Iwatsuki, K. \& M. Kato. 1980. Enumeration of Kalimantan pteridophytes collected during 1978-1979 (1). Acta Phytotax. Geobot. 31: 24-43.

Jacquin, N. 1789. Collectanea, vol. 3. Publisher unknown, Vienna.

Klotzsch, J.F. 1844. Beiträge zu einer Flora der Aequinoctial-Gegenden der neuen Welt. Linnaea 18: 525-539.

Kramer, K.U. 1978. The pteridophytes of Suriname: An enumeration with keys of the ferns and fernallies. Natuurwetenschappelijke Studiekring voor Suriname en de Nederlandse Antillen, Utrecht. 
Kuhn, F.A.M. 1879. Cryptogame vasculares. In: C.C. von der Decken (ed.), Reisen in Ost-Afrika, Bd. 3, Abth. 3: 7-71. Winter, Leipzig.

Kunth, K.S. 1815. Nova Genera et Species Plantarum. Publisher unknown, Paris.

Kunze, G. 1837. Analecta Pteridographica. Publisher unknown, Leipzig.

Lellinger, D. B. 1989. The ferns and fern-allies of Costa Rica, Panama, and the Chocó (Part 1: Psilotaceae through Dicksoniaceae). Pteridologia 2A: 1-364.

Linnaeus, C. 1753. Species Plantarum, vol. 2. Impensis Laurentii Salvii, Stockholm.

Makino, T. 1899. Plantae Japonenses novae vel minus cognitae. Bot. Mag. (Tokyo) 13: 44-48.

Marticorena, C. \& R. Rodriguez. 1995. Flora de Chile, Vol. I. Pteridophyta - Gymnospermae. Universidad de Concepción, Concepción.

Martius, C.F.P. 1859. Flora Brasiliensis, vol. 12. Fleischer, Leipzig.

Mettenius, G.H. 1864. Ueber die Hymenophyllaceae. Publisher unknown, Leipzig.

Mickel, J.T. \& J.B. Beitel. 1988. Pteridophyte flora of Oaxaca, Mexico. Mem. New York Bot. Gard. 46: $1-568$.

Moore, T. 1857. Index Filicum. Pamplin, London.

Moore, T. 1862. Diagnoses speciarum novarum in hortis cultivarum interdum sub titulo: new garden ferns. Gard. Chron. ser. 2: 44-45.

Morton, C.V. 1932. Buesia, a new subgenus of Hymenophyllum from Peru. Bot. Gaz. 93: 336339.

Morton, C.V. 1947. The American species of Hymenophyllum section Sphaerocionium. Contr. U.S. Natl. Herb. 29: 139-202.

Morton, C.V. 1968. The genera, subgenera, and sections of the Hymenophyllaceae. Contr. U.S. Natl. Herb. 38: 153-214.

Morton, C.V. 1973. Studies of fern types, II. Contr. U.S. Natl. Herb. 38: 215-281.

Müller, K. 1854. Ueber einige bisher verwechselte Arten der Farrngruppe der Hymenophyllaceae IV-VI. Bot. Zeitung (Berlin) 12: 729-738.

Nadeaud, J. 1873. Énumération des plantes indigènes de l'Ile de Tahiti. Librairie de la Société Botanique de France, Paris.

Nakai, T. 1926. Tentamen systematis Hymenophyllacearum Japonicarum. Bot. Mag. (Tokyo) 40: 239-275.

Nakaike, T. 1975. Enumeratio Pteridophytarum Japonicarum - Filicales. University of Tokyo Press, Tokyo.

Pacheco, L. 1995. Hymenophyllaceae. In: G. Davidse, S. Sousa, C. Mario \& S. Knapp (eds.), Flora Mesoamericana Vol. 1: Psilotaceae a Salviniaceae: 62-83. Universidad Nacional Autónoma de México, Instituto de Biologia, México, D.F.

Parris, B.S. 1992. The plants of Mount Kinabalu 1: Ferns and fern allies. Royal Botanic Gardens Kew, London.

Philippi, R.A. 1860. Plantarum novarum Chilensium. Centuriae sextae continuatio. Linnaea 30: $185-212$.

Pichi Sermolli, E.G. 1970. Fragmenta pteridologiae II. Webbia 24: 699-722.

Pichi Sermolli, E.G. 1973. Fragmenta pteridologiae IV. Webbia 28: 445-477.

Pichi Sermolli, E.G. 1977a. Fragmenta pteridologiae VI. Webbia 31: 237-259.

Pichi Sermolli, E. G. 1977b. Tentamen pteridophytorum genera in taxonomicum ordinem redigendi. Webbia 31: 315-512.

Pichi Sermolli, E.G. 1981. The controversial typification of the Linnaean genus Trichomanes (Hymenophyllaceae). Taxon 30: 809-815.

Pichi Sermolli, E.G. 1983. A contribution to the knowledge of the Pteridophyta of Rwanda, Burundi, and Kivu (Zaire) I. Bull. Jard. Bot. Belg. 53: 177-284.

Prantl, K.A.E. 1875. Die Hymenophyllaceen, die niedrigste Entwicklungsreihe der Farne. Unters. Morph. Gefasskrypt. 1: 1-73, pl. 1-6.

Presl, C.B. 1843. Hymenophyllaceae. Haase, Prague.

Presl, C.B. 1848. Die Gefässbündel im Stipes der Farrn. Abh. Königl. Böhm. Ges. Wiss. 5, 5: 307356, pl. 1-7.

Presl, C.B. 1849. Epimeliae Botanicae. Haase, Prague. 
Proctor, G. R. 1985. Ferns of Jamaica: A guide to the pteridophytes. British Museum (Natural History), London.

Proctor, G.R. 1989. Ferns of Puerto Rico and the Virgin Islands. Mem. New York Bot. Gard. 53: $1-389$.

Pryer, K.M., A.R. Smith, J.S. Hunt \& J.-Y. Dubuisson. 2001. rbcL data reveal two monophyletic groups of filmy ferns (Filicopsida: Hymenophyllaceae). Amer. J. Bot. 88: 1118-1130.

Raddi, G. 1825. Plantarum Brasiliensium Nova Genera. Pezzati, Firenze.

Reed, C.F. 1948. Two new generic names of ferns. Amer. Fern J. 28: 87-89.

Reichenbach, H.G.L. 1841. Deutsche Botaniker. Erster Band. Das Herbarienbuch. Dresden, Leipzig.

Reinwardt, C.G.C. 1825. Verbesserungen und Druckfehler. Flora 8, 2 Beibl. 3: 47-48.

Rosenstock, E. 1908a. Filices Novo-Guineenses novae. Repert. Spec. Nov. Regni Veg. 5: 33-44.

Rosenstock, E. 1908b. Filices Novo-Guineenses novae. Repert. Spec. Nov. Regni Veg. 5: 370376.

Sánchez, C. 2000. Flora de la República de Cuba, Serie A. Plantas vasculares, Fasciculo 4, Hymenophyllaceae. Koeltz Scientific, Königstein.

Satou, Z. 1997. Miscellaneous notes on ferns and fern allies (1-4). Hikobia 12: 267-270.

Schneider, H. 2000. Morphology and anatomy of roots in the filmy fern tribe Trichomaneae H. Schneider (Hymenophyllaceae, Filicatae) and the evolution of rootless taxa. Bot. J. Linn. Soc. 132: 29-46.

Schuettpelz, E. \& K.M. Pryer. In press. Reconciling extreme branch length differences: decoupling time and rate through the evolutionary history of filmy ferns. Syst. Bot.

Smith, A.R. 1981. Flora of Chiapas Part 2, Pteridophytes. California Academy of Sciences, San Francisco.

Smith, J. 1875. Historia Filicum. Macmillan, London.

Smith, J.E. 1793. Tentamen botanicum de filicum generibus dorsiferarum. Mem. Acad. Sci. Turin 5: 401-422.

Spach, É. 1839. Histoire naturelle des végétaux phanérogames. Vol. 7.

Stolze, R.G. 1976. Ferns and fern allies of Guatemala I, Ophioglossaceae through Cyatheaceae. Fieldiana, Bot. 39: 1-130.

Sturm, J. W. 1858. Enumeratio plantarum vascularium cryptogamicarum Chilensium. Abh. Naturhist. Ges. Nürnberg 1: 151-202.

Swartz, O.P. 1788. Nova Genera \& Species Plantarum seu Prodromus Descriptionum Vegetabilium, Maximam Partem Incognitorum quae sub Itinere in Indiam Occidentalem Annis 1783-1787. Bibliopoliis Acad. Swederi, Stockholm.

Tagawa, M. 1939. Specilegium pteridographiae Asiae Orientalis. Acta Phytotax. Geobot. 8: 164176.

Tagawa, M. \& K. Iwatsuki. 1979. Flora of Thailand, Vol. 3, part 1. Forest Herbarium, Royal Forest Department, Bangkok.

Tardieu-Blot, M.L. 1977. Sur quelques Hymenophyllaceae des îles Mascareignes. Adansonia, n.s. 17: $147-150$.

Torrey, J. \& A. Gray. 1840. A Flora of North America. Vol. 1. Wiley \& Putnam, New York.

Tryon, R.M. \& R.G. Stolze. 1989. Pteridophyta of Peru, Part I. Fieldiana, Bot., n.s. 20: 1-145.

Tryon, R.M. \& A.F. Tryon. 1981. Taxonomic and nomenclatural notes on ferns. Rhodora 83: $133-137$.

Tryon, R.M. \& A.F. Tryon. 1982. Ferns and allied plants with special reference to Tropical America. Springer Verlag, New York.

Van Alderwerelt van Rosenburgh, C.R.W.K. 1924. Pteridophyta. Nova Guinea 14: 1-68.

Van den Bosch, R. B. 1859. Synopsis Hymenophyllacearum. Ned. Kruidk. Arch. 4: 341-419.

Van den Bosch, R.B. 1861a. Eerste Bijdrage tot de Kennis der Hymenophyllaceae. Verslagen Meded. Afd. Natuurk. Kon. Akad. Wetensch. 11: 300-330.

Van den Bosch, R.B. 1861b. Hymenophyllaceae novas, synopseos supplementum. Ned. Kruidk. Arch. 5, 2: 135-185. 
Van den Bosch, R.B. 1861c. Hymenophyllaceae Novae Caledoniae. Ann. Sci. Nat., Bot. sér. 4, 15: $88-91$.

Van den Bosch, R.B. 1863. Hymenophyllaceae novas. Ned. Kruidk. Arch. 5, 3: 135-217.

Wallich, N. 1832. Plantae Asiaticae Rariores, vol. 3. Treuttel, Wurtz \& Richter, London.

Wessels Boer, J.G. 1962. The New World species of Trichomanes sect. Didymoglossum and Microgonium. Acta Bot. Neerl. 11: 227-330.

Willdenow, C.L. 1810. Species Plantarum. Editio Quarta. Impensis G.C. Nauk, Berlin.

Yoroi, R. \& K. Iwatsuki. 1977. An observation on the variation of Trichomanes minutum and allied species. Acta Phytotax. Geobot. 28: 152-159. 
Appendix. Taxonomic placement (genus, subgenus and section) of each basionym of Hymenophyllaceae (excl. later homonyms) in the present classification.

\begin{tabular}{|c|c|c|}
\hline Basionym & Genus & Subgenus / Section \\
\hline \multicolumn{3}{|l|}{ Abrodictyum } \\
\hline boninense Tagawa \& K. Iwats. & Abrodictyum & Abrodictyum \\
\hline cumingii C. Presl & Abrodictyum & Abrodictyum \\
\hline \multicolumn{3}{|l|}{ Adiantum } \\
\hline decurrens Jacq. & Hymenophyllum & Mecodium \\
\hline tenellum Jacq. & Hymenophyllum & Hymenophyllum \\
\hline trifoliatum L. & Hymenophyllum & $?$ \\
\hline \multicolumn{3}{|l|}{ Amphipterum } \\
\hline humatoides Copel. & Hymenophyllum & Hymenophyllum \\
\hline \multicolumn{3}{|l|}{ Apteropteris } \\
\hline applanata A.M. Gray \& R.G. Williams & Hymenophyllum & Sphaerocionium \\
\hline \multicolumn{3}{|l|}{ Buesia } \\
\hline megistocarpa Copel. & Hymenophyllum & Hymenophyllum \\
\hline \multicolumn{3}{|l|}{ Callistopteris } \\
\hline calyculata Copel. & Callistopteris & - \\
\hline mulensis K. Iwats. & Abrodictyum & Abrodictyum \\
\hline \multicolumn{3}{|l|}{ Cephalomanes } \\
\hline atrovirens C. Presl & Cephalomanes & - \\
\hline australicum Bosch & Cephalomanes & - \\
\hline curvatum Bosch & Cephalomanes & - \\
\hline madagascariense Bosch & Trichomanes & $?$ \\
\hline oblongifolium C. Presl & Cephalomanes & - \\
\hline rhomboideum Bosch & Cephalomanes & - \\
\hline singaporianum Bosch & Cephalomanes & - \\
\hline wilkesii Bosch & Cephalomanes & - \\
\hline \multicolumn{3}{|l|}{ Crepidomanes } \\
\hline agasthianum Madhus. \& C.A. Hameed & Crepidomanes & Crepidomanes/Crepidomanes \\
\hline chuii Ching \& P.C. Chiu & Crepidomanes & Crepidomanes/Crepidomanes \\
\hline dilatatum Ching \& Chu H. Wang & Crepidomanes & Crepidomanes/Crepidomanes \\
\hline hainanense Ching & Crepidomanes & Crepidomanes/Crepidomanes \\
\hline indicum C.A. Hameed \& Madhus. & Crepidomanes & Crepidomanes/Crepidomanes \\
\hline liboense P.S. Wang & Crepidomanes & Crepidomanes/Crepidomanes \\
\hline lunulatum Madhus. \& C.A. Hameed & Crepidomanes & Crepidomanes/Crepidomanes \\
\hline malabaricum C.A. Hameed \& Madhus. & Crepidomanes & Crepidomanes/Crepidomanes \\
\hline nanophyllum Tagawa & Crepidomanes & Crepidomanes/Crepidomanes \\
\hline omeiense Ching \& P.C. Chiu & Crepidomanes & Crepidomanes/Crepidomanes \\
\hline paucinervium Ching & Crepidomanes & Crepidomanes/Crepidomanes \\
\hline pinnatifidum Ching \& P.C. Chiu & Crepidomanes & Crepidomanes/Crepidomanes \\
\hline pseudonymanii Hosok. & Crepidomanes & Crepidomanes/Crepidium? \\
\hline sarawakense $\mathrm{K}$. Iwats. & Crepidomanes & Crepidomanes/Crepidomanes \\
\hline smithiae Ching & Crepidomanes & Crepidomanes/Crepidomanes \\
\hline tagawanum $\mathrm{K}$. Iwats. & Crepidomanes & Crepidomanes/Crepidomanes \\
\hline tiendongense Ching \& C.F. Zhang & Crepidomanes & Crepidomanes/Crepidomanes \\
\hline yunnanense Ching \& P.C. Chiu & Crepidomanes & Crepidomanes/Crepidomanes \\
\hline zayüense Ching \& S.K. Wu & Crepidomanes & Crepidomanes/Crepidomanes \\
\hline \multicolumn{3}{|l|}{ Davallia } \\
\hline tegularis Desv. & Hymenophyllum & $?$ \\
\hline \multicolumn{3}{|l|}{ Didymoglossum } \\
\hline acanthoides Bosch & Hymenophyllum & Hymenophyllum \\
\hline aculeatum Bosch & Hymenophyllum & Hymenophyllum \\
\hline affine Bosch & Hymenophyllum & Hymenophyllum \\
\hline angustifrons Fée & Didymoglossum & Didymoglossum \\
\hline
\end{tabular}


Appendix. Continued.

\begin{tabular}{|c|c|c|}
\hline Basionym & Genus & Subgenus / Section \\
\hline \multicolumn{3}{|l|}{ Didymoglossum (cont.) } \\
\hline anomalum Bosch & Crepidomanes & Crepidomanes/Crepidomanes \\
\hline braunii Bosch & Hymenophyllum & Hymenophyllum \\
\hline brevipes C. Presl & Crepidomanes & Crepidomanes/Crepidomanes \\
\hline cordifolium Fée & Didymoglossum & Didymoglossum \\
\hline decipiens Desv. & Polyphlebium & - \\
\hline dilatatum Bosch & Crepidomanes & Crepidomanes/Crepidomanes \\
\hline euphlebium Bosch & Crepidomanes & Crepidomanes/Crepidomanes \\
\hline ferox Hassk. & Hymenophyllum & Hymenophyllum \\
\hline fructuosum Fée & Didymoglossum & Didymoglossum \\
\hline griffithii Bosch & Crepidomanes & Crepidomanes/Crepidomanes \\
\hline hedwigii C. Presl & Didymoglossum & Didymoglossum \\
\hline holochilum Bosch & Hymenophyllum & Hymenophyllum \\
\hline hookeri C. Presl & Didymoglossum & Didymoglossum \\
\hline insigne Bosch & Crepidomanes & Crepidomanes/Crepidomanes \\
\hline laceratum Fée & Didymoglossum & Didymoglossum \\
\hline latealatum Bosch & Crepidomanes & Crepidomanes/Crepidomanes \\
\hline lineolatum Bosch & Didymoglossum & Didymoglossum \\
\hline longisetum C. Presl & Abrodictyum & Pachychaetum \\
\hline magellanicum Desv. & Hymenophyllum & Myrmecostylum \\
\hline nummularium Bosch & Didymoglossum & Didymoglossum \\
\hline ovale E. Fourn. & Didymoglossum & Didymoglossum \\
\hline palmarum Vareschi & Didymoglossum & Didymoglossum \\
\hline plicatum Bosch & Crepidomanes & Crepidomanes/Crepidomanes \\
\hline preslii Bosch & Hymenophyllum & Hymenophyllum \\
\hline racemulosum Bosch & Crepidomanes & Crepidomanes/Crepidomanes \\
\hline serrulatum C. Presl & Hymenophyllum & Hymenophyllum \\
\hline sociale Fée & Didymoglossum & Didymoglossum \\
\hline undulatum C. Presl & Crepidomanes & Crepidomanes/Crepidomanes \\
\hline wesselsboeri Ebihara \& Dubuisson & Didymoglossum & Microgonium \\
\hline \multicolumn{3}{|l|}{ Feea } \\
\hline boryi Bosch & Trichomanes & Feea \\
\hline humboldti Bosch & Trichomanes & Trichomanes \\
\hline nana Bory & Trichomanes & Feea \\
\hline polypodina Bory & Trichomanes & Feea \\
\hline \multicolumn{3}{|l|}{ Gonocormus } \\
\hline australis Ching & Crepidomanes & Crepidomanes/Gonocormus \\
\hline samoensis Copel. & Crepidomanes & Crepidomanes/Gonocormus \\
\hline siamensis Tagawa \& K. Iwats. & Crepidomanes & Crepidomanes/Gonocormus \\
\hline \multicolumn{3}{|l|}{ Hymenophyllum } \\
\hline abietinum Hook. \& Grev. & Hymenophyllum & Mecodium \\
\hline abruptum Hook. & Hymenophyllum & Mecodium \\
\hline acrosorum Bosch & Hymenophyllum & Mecodium \\
\hline aculeolatum Bosch & Hymenophyllum & Hymenophyllum? \\
\hline adiantoides Bosch & Hymenophyllum & Sphaerocionium \\
\hline aequabile Kunze & Hymenophyllum & Sphaerocionium \\
\hline affine Brack. & Hymenophyllum & Hymenophyllum \\
\hline alatum $\mathrm{Sm}$. & Vandenboschia? & Vandenboschia \\
\hline alfredii Rosenst. & Hymenophyllum & Mecodium \\
\hline alishanense De Vol & Hymenophyllum & Hymenophyllum \\
\hline alpinum Colenso & Hymenophyllum & Hymenophyllum \\
\hline alternatum Fosberg & Hymenophyllum & Sphaerocionium \\
\hline alveolatum C. Chr. & Hymenophyllum & Hymenophyllum? \\
\hline
\end{tabular}


Appendix. Continued.

\begin{tabular}{|c|c|c|}
\hline Basionym & Genus & Subgenus / Section \\
\hline \multicolumn{3}{|l|}{ Hymenophyllum (cont.) } \\
\hline amabile C.V. Morton & Hymenophyllum & Sphaerocionium \\
\hline amoenum Sturm & Hymenophyllum & Sphaerocionium \\
\hline andinum Bosch & Hymenophyllum & Mecodium \\
\hline angulosum $\mathrm{H}$. Christ & Hymenophyllum & Globosa \\
\hline angustatum Kunze & Hymenophyllum & Sphaerocionium \\
\hline angustifrons $\mathrm{H}$. Christ & Hymenophyllum & Sphaerocionium \\
\hline angustum Bosch & Hymenophyllum & Sphaerocionium \\
\hline anisopterum Peter & Hymenophyllum & Sphaerocionium \\
\hline antarcticum C. Presl & Hymenophyllum & Hymenophyllum \\
\hline apicale Bosch & Hymenophyllum & Mecodium \\
\hline apiculatum Kuhn & Hymenophyllum & Mecodium \\
\hline apterum Bosch & Hymenophyllum & Sphaerocionium \\
\hline arbuscula Desv. & Hymenophyllum & Sphaerocionium \\
\hline asperulum Kunze & Hymenophyllum & Hymenophyllum \\
\hline assamense Gand. & Hymenophyllum & Globosa \\
\hline asterothrix Kunze & Hymenophyllum & Sphaerocionium \\
\hline atrosanguineum Bosch & Hymenophyllum & Mecodium \\
\hline atrovirens Colenso & Hymenophyllum & Globosa \\
\hline attenuatum Hook. & Hymenophyllum & Myrmecostylum \\
\hline aucklandicum Bosch & Hymenophyllum & Globosa \\
\hline australe Willd. & Hymenophyllum & Globosa \\
\hline austrosinicum Ching & Hymenophyllum & Hymenophyllum \\
\hline axillare $\mathrm{Sw}$. & Hymenophyllum & Mecodium \\
\hline babindae Watts & Hymenophyllum & Hymenophyllum \\
\hline badium Hook. \& Grev. & Hymenophyllum & Globosa \\
\hline baileyanum Domin & Hymenophyllum & Hymenophyllum \\
\hline bakeri Copel. & Hymenophyllum & Hymenophyllum \\
\hline balansae E. Fourn. & Hymenophyllum & Globosa \\
\hline baldwinii D.C. Eaton & Callistopteris & - \\
\hline balfourii Baker & Hymenophyllum & Mecodium \\
\hline bamlerianum Rosenst. & Hymenophyllum & Globosa \\
\hline batuense Rosenst. & Hymenophyllum & Hymenophyllum \\
\hline berteroi Hook. & Hymenophyllum & Sphaerocionium \\
\hline beyrichianum Kunze & Hymenophyllum & Sphaerocionium \\
\hline bibraianum Sturm & Hymenophyllum & Myrmecostylum \\
\hline bicolanum Copel. & Hymenophyllum & Hymenophyllum \\
\hline bismarckianum $\mathrm{H}$. Christ & Hymenophyllum & Globosa \\
\hline blandum Racib. & Hymenophyllum & Hymenophyllum \\
\hline blepharodes C. Presl & Hymenophyllum & Hymenophyllum \\
\hline blumeanum A. Spreng. & Hymenophyllum & Mecodium \\
\hline bontocense Copel. & Hymenophyllum & Hymenophyllum \\
\hline borneense Hook. & Hymenophyllum & Sphaerocionium \\
\hline boryanum Bory ex Willd. & Hymenophyllum & Sphaerocionium \\
\hline boschii Rosenst. & Hymenophyllum & Hymenophyllum \\
\hline botryoides Bosch & Hymenophyllum & Mecodium \\
\hline bougainvillense Jeff W. Grimes & Hymenophyllum & Hymenophyllum \\
\hline boutoni Baker & Hymenophyllum & Sphaerocionium \\
\hline brachyglossum A. Braun ex Kunze & Hymenophyllum & Hymenophyllum \\
\hline brachypus Sodiro & Hymenophyllum & Sphaerocionium? \\
\hline braithwaitei Ebihara \& K. Iwats. & Hymenophyllum & Sphaerocionium \\
\hline brassii C. Chr. & Hymenophyllum & Hymenophyllum \\
\hline breve Rosenst. & Hymenophyllum & Mecodium? \\
\hline
\end{tabular}


Appendix. Continued.

\begin{tabular}{|c|c|c|}
\hline Basionym & Genus & Subgenus / Section \\
\hline \multicolumn{3}{|l|}{ Hymenophyllum (cont.) } \\
\hline brevidens Alderw. & Hymenophyllum & Hymenophyllum \\
\hline brevifrons Kunze & Hymenophyllum & Mecodium \\
\hline brevistipes Liebm. & Hymenophyllum & Mecodium \\
\hline bridgesii Hook. & Hymenophyllum & Hymenophyllum \\
\hline bryophilum C. Chr. & Hymenophyllum & Hymenophyllum \\
\hline buchtienii Rosenst. & Hymenophyllum & Sphaerocionium \\
\hline caespitosum Gaudich. & Hymenophyllum & Hymenophyllum \\
\hline calodictyon Bosch & Hymenophyllum & Hymenophyllum \\
\hline campanulatum $\mathrm{H}$. Christ & Hymenophyllum & Hymenophyllum \\
\hline caparaoense Brade & Hymenophyllum & Sphaerocionium \\
\hline capense Schrad. & Hymenophyllum & Mecodium \\
\hline capillaceum Roxb. & Hymenophyllum & Mecodium? \\
\hline capillare Desv. & Hymenophyllum & Sphaerocionium \\
\hline capurroi de la Sota & Hymenophyllum & Sphaerocionium \\
\hline cardunculus C. Chr. & Hymenophyllum & Hymenophyllum \\
\hline carnosum $\mathrm{H}$. Christ & Hymenophyllum & Mecodium \\
\hline catherinae Hook. & Hymenophyllum & Sphaerocionium \\
\hline caudatellum $\mathrm{H}$. Christ & Hymenophyllum & Sphaerocionium \\
\hline caudatum Bosch & Hymenophyllum & Globosa \\
\hline caudiculatum Mart. & Hymenophyllum & Globosa \\
\hline caulopteron Fée & Hymenophyllum & Sphaerocionium \\
\hline ceratophylloides $\mathrm{H}$. Christ & Hymenophyllum & Sphaerocionium \\
\hline cеrnuит A. Gepp & Hymenophyllum & Hymenophyllum \\
\hline cheesemannii Baker & Hymenophyllum & Hymenophyllum \\
\hline chiloense Hook. & Hymenophyllum & Hymenophyllum \\
\hline chrysothrix Sturm & Hymenophyllum & Sphaerocionium \\
\hline cincinnatum A. Gepp & Hymenophyllum & Hymenophyllum \\
\hline clemensiae Copel. & Hymenophyllum & Hymenophyllum \\
\hline cocosense A. Rojas & Hymenophyllum & Sphaerocionium \\
\hline coloratum A. Braun ex Bosch & Hymenophyllum & Mecodium \\
\hline compactum Bonap. & Hymenophyllum & Mecodium \\
\hline consanguineum C.V. Morton & Hymenophyllum & Sphaerocionium \\
\hline constrictum $\mathrm{H}$. Christ & Hymenophyllum & Sphaerocionium \\
\hline contextum Rosenst. & Hymenophyllum & Mecodium \\
\hline contortum Bosch & Hymenophyllum & Mecodium \\
\hline contractile Sodiro & Hymenophyllum & Sphaerocionium \\
\hline convolutum Bosch & Hymenophyllum & Mecodium \\
\hline copelandianum Alderw. & Hymenophyllum & Globosa \\
\hline copelandii C.V. Morton & Hymenophyllum & Mecodium \\
\hline coreanum Nakai & Hymenophyllum & Mecodium \\
\hline corrugatum $\mathrm{H}$. Christ & Hymenophyllum & Mecodium \\
\hline costaricanum Bosch & Hymenophyllum & Mecodium \\
\hline crassipetiolatum Stolze & Hymenophyllum & Sphaerocionium \\
\hline crispato-alatum Hayata & Hymenophyllum & Globosa \\
\hline crispatulum Bosch & Hymenophyllum & Sphaerocionium \\
\hline crispatum Wall. ex Hook. \& Grev. & Hymenophyllum & Globosa \\
\hline crispum Kunth & Hymenophyllum & Sphaerocionium \\
\hline cristatum Hook. \& Grev. & Hymenophyllum & Hymenophyllum \\
\hline cristulatum Rosenst. & Hymenophyllum & Myrmecostylum \\
\hline cruegeri Müll.Berol. & Hymenophyllum & Sphaerocionium \\
\hline cruentum Cav. & Hymenophyllum & Hymenoglossum \\
\hline cubense Sturm & Hymenophyllum & Sphaerocionium \\
\hline
\end{tabular}


Appendix. Continued.

\begin{tabular}{|c|c|c|}
\hline Basionym & Genus & Subgenus / Section \\
\hline \multicolumn{3}{|l|}{ Hymenophyllum (cont.) } \\
\hline cumingii C. Presl & Hymenophyllum & Mecodium \\
\hline cuneatum Kunze & Hymenophyllum & Mecodium \\
\hline cupressiforme Labill. & Hymenophyllum & Hymenophyllum \\
\hline daedaleum Blume & Hymenophyllum & Globosa \\
\hline darwinii Hook. & Hymenophyllum & Mecodium \\
\hline dejectum Baker & Hymenophyllopsis & - \\
\hline delavayi $\mathrm{H}$. Christ & Hymenophyllum & Hymenophyllum \\
\hline delicatissimum Fée & Hymenophyllum & Sphaerocionium \\
\hline delicatulum Sehnem & Hymenophyllum & Sphaerocionium \\
\hline deltoideum C. Chr. & Hymenophyllum & Hymenophyllum \\
\hline dendritis Rosenst. & Hymenophyllum & Mecodium \\
\hline densifolium Phil. & Hymenophyllum & Hymenophyllum \\
\hline dentatum Cav. & Hymenophyllum & Hymenophyllum \\
\hline denticulatum Sw. & Hymenophyllum & Hymenophyllum \\
\hline dependens C.V. Morton & Hymenophyllum & Sphaerocionium \\
\hline deplanchei Mett. ex Kuhn & Hymenophyllum & Hymenophyllum \\
\hline dichotomum Cav. & Hymenophyllum & Hymenophyllum \\
\hline dimidiatum Mett. ex Kuhn & Hymenophyllum & Hymenophyllum \\
\hline dimorphum $\mathrm{H}$. Christ & Hymenophyllum & Sphaerocionium \\
\hline dipteroneuron A. Braun ex Kunze & Hymenophyllum & Hymenophyllum \\
\hline discosum $\mathrm{H}$. Christ & Hymenophyllum & Mecodium \\
\hline divaricatum Bosch & Hymenophyllum & Sphaerocionium \\
\hline dregeanum C. Presl & Hymenophyllum & Hymenophyllum \\
\hline durandi $\mathrm{H}$. Christ & Hymenophyllum & Sphaerocionium \\
\hline dusenii H. Christ & Hymenophyllum & Hymenophyllum \\
\hline eboracense Croxall & Hymenophyllum & Globosa \\
\hline ectocarpon Fée & Hymenophyllum & Hymenophyllum \\
\hline elasticum Bory ex Willd. & Hymenophyllum & Sphaerocionium \\
\hline elatius H. Christ & Hymenophyllum & Sphaerocionium \\
\hline elberti Rosenst. & Hymenophyllum & Hymenophyllum \\
\hline elegans A. Spreng. & Hymenophyllum & Sphaerocionium \\
\hline elegantissimum Fée & Hymenophyllum & Sphaerocionium \\
\hline elegantulum Bosch & Hymenophyllum & Sphaerocionium \\
\hline ellipticosorum Alderw. & Hymenophyllum & Hymenophyllum \\
\hline elongatum Jeff W. Grimes & Hymenophyllum & Hymenophyllum \\
\hline emarginatum $\mathrm{Sw}$ & Hymenophyllum & Globosa \\
\hline emersum Baker & Hymenophyllum & Hymenophyllum \\
\hline endiviaefolium Desv. & Hymenophyllum & Mecodium \\
\hline epiphyticum J.W. Moore & Hymenophyllum & Mecodium \\
\hline erecto-alatum Colenso & Hymenophyllum & Globosa \\
\hline eriophorum Bosch & Hymenophyllum & Sphaerocionium \\
\hline erosum Blume & Hymenophyllum & Globosa \\
\hline exiguum Bedd. & Didymoglossum & Didymoglossum \\
\hline eximium Kunze & Hymenophyllum & Globosa \\
\hline exsertum Wall. ex Hook. & Hymenophyllum & Hymenophyllum \\
\hline falklandicum Baker & Hymenophyllum & Hymenophyllum \\
\hline farallonense Hieron. & Hymenophyllum & Mecodium \\
\hline fastigiosum $\mathrm{H}$. Christ & Hymenophyllum & Hymenophyllum \\
\hline fecundum Bosch & Hymenophyllum & Mecodium \\
\hline feejeense Brack. & Hymenophyllum & Hymenophyllum \\
\hline fendlerianum Sturm & Hymenophyllum & Mecodium \\
\hline ferax Bosch & Hymenophyllum & Mecodium \\
\hline
\end{tabular}


Appendix. Continued.

\begin{tabular}{|c|c|c|}
\hline Basionym & Genus & Subgenus / Section \\
\hline \multicolumn{3}{|l|}{ Hymenophyllum (cont.) } \\
\hline ferrugineum Colla & Hymenophyllum & Sphaerocionium \\
\hline filicula Bory ex Willd. & Crepidomanes & Crepidomanes/Crepidomanes \\
\hline fimbriatum J. Sm. ex Hook. & Hymenophyllum & Globosa \\
\hline firmum Alderw. & Hymenophyllum & Hymenophyllum \\
\hline flabellatum Labill. & Hymenophyllum & Pleuromanes \\
\hline flaccidum Bosch & Hymenophyllum & Mecodium \\
\hline flavo-aureum Bory & Hymenophyllum & Sphaerocionium \\
\hline flexile Makino & Hymenophyllum & Globosa \\
\hline flexuosum A. Cunn. & Hymenophyllum & Globosa \\
\hline floribundum Kunth & Hymenophyllum & Mecodium? \\
\hline foersteri Rosenst. & Hymenophyllum & Hymenophyllum \\
\hline formosum Brack. & Hymenophyllum & Globosa \\
\hline foxworthyi Copel. & Hymenophyllum & Pleuromanes \\
\hline francavillei Bosch & Hymenophyllum & Sphaerocionium \\
\hline frankliniae Colenso & Hymenophyllum & Sphaerocionium \\
\hline franklinianum Colenso & Hymenophyllum & Sphaerocionium \\
\hline fraseri Mett. & Hymenophyllum & Sphaerocionium \\
\hline fraternum C. Presl & Hymenophyllum & Mecodium \\
\hline fuciforme Sw. & Hymenophyllum & Fuciformia \\
\hline fucoides Cav. & Hymenophyllum & Fuciformia \\
\hline fuertesii Brause & Hymenophyllum & Sphaerocionium \\
\hline fujisanense Nakai & Hymenophyllum & Mecodium \\
\hline fulvum Bosch & Hymenophyllum & Sphaerocionium \\
\hline fumarioides Bory ex Willd. & Hymenophyllum & Mecodium \\
\hline funckii Bosch & Hymenophyllum & Mecodium \\
\hline fusugasugense H. Karst. ex Sturm & Hymenophyllum & Sphaerocionium \\
\hline gardneri Bosch & Hymenophyllum & Hymenophyllum \\
\hline gardnerianum Sturm & Hymenophyllum & Sphaerocionium \\
\hline geluense Rosenst. & Hymenophyllum & Hymenophyllum \\
\hline glaziovii Baker & Hymenophyllum & Sphaerocionium \\
\hline glebarium $\mathrm{H}$. Christ & Hymenophyllum & Hymenophyllum \\
\hline gollmeri Bosch & Hymenophyllum & Mecodium \\
\hline gorgoneum Copel. & Hymenophyllum & Hymenophyllum \\
\hline gracile Bory ex Willd. & Hymenophyllum & Mecodium \\
\hline gracilescens Domin & Hymenophyllum & Hymenophyllum \\
\hline gracilius Copel. & Hymenophyllum & Mecodium \\
\hline gratum Fée & Hymenophyllum & Sphaerocionium \\
\hline grevilleanum $\mathrm{C}$. Presl & Hymenophyllum & Mecodium \\
\hline gunnii Bosch ex Baker & Hymenophyllum & Mecodium \\
\hline haematochroum Sodiro & Hymenophyllum & Hymenophyllum \\
\hline halconense Copel. & Hymenophyllum & Hymenophyllum \\
\hline hallieri Rosenst. & Hymenophyllum & Hymenophyllum \\
\hline hamuliferum Alderw. & Hymenophyllum & Hymenophyllum \\
\hline hayatai Masam. & Hymenophyllum & Mecodium \\
\hline heimii Tardieu & Hymenophyllum & Hymenoglossum \\
\hline helicoideum Sodiro & Hymenophyllum & Mecodium \\
\hline hemidimorphum R.C. Moran \& B. Øllg. & Hymenophyllum & Sphaerocionium \\
\hline hemipteron Rosenst. & Hymenophyllum & Sphaerocionium \\
\hline henkelii Sim & Hymenophyllum & Mecodium \\
\hline henryi Baker & Hymenophyllum & Hymenophyllum \\
\hline l'herminieri Mett. ex Kuhn & Hymenophyllum & Mecodium? \\
\hline herterianum Brause & Hymenophyllum & Hymenophyllum \\
\hline
\end{tabular}


Appendix. Continued.

\begin{tabular}{|c|c|c|}
\hline Basionym & Genus & Subgenus / Section \\
\hline \multicolumn{3}{|l|}{ Hymenophyllum (cont.) } \\
\hline herzogii Rosenst. & Hymenophyllum & Mecodium \\
\hline himalaianum Bosch & Hymenophyllum & Mecodium \\
\hline hirtellum Sw. & Hymenophyllum & Sphaerocionium \\
\hline holotrichum Peter & Hymenophyllum & Sphaerocionium \\
\hline hookeri Bory & Hymenophyllum & Pleuromanes \\
\hline horizontale C.V. Morton & Hymenophyllum & Sphaerocionium \\
\hline hosei Copel. & Hymenophyllum & Hymenophyllum \\
\hline houstonii Jenman & Hymenophyllum & Hymenophyllum \\
\hline howense Brownlie & Hymenophyllum & Hymenophyllum \\
\hline humbertii C. Chr. & Hymenophyllum & Hymenophyllum \\
\hline humboldtianum E. Fourn. & Hymenophyllum & Globosa \\
\hline humile Nees \& Blume & Hymenophyllum & Hymenophyllum \\
\hline imbricatum Blume & Hymenophyllum & Globosa \\
\hline inclinatum Bosch & Hymenophyllum & Globosa \\
\hline infortunatum Bory & Hymenophyllum & Mecodium \\
\hline integrivalvatum C. Sánchez & Hymenophyllum & Hymenophyllum \\
\hline integrum Bosch & Hymenophyllum & Mecodium \\
\hline intercalatum $\mathrm{H}$. Christ & Hymenophyllum & Sphaerocionium \\
\hline interruptum Kunze & Hymenophyllum & Sphaerocionium \\
\hline intricatum Bosch & Hymenophyllum & Globosa \\
\hline involucratum Copel. & Hymenophyllum & Mecodium \\
\hline ivohibense Tardieu & Hymenophyllum & Sphaerocionium \\
\hline jalapense Schltdl. \& Cham. & Hymenophyllum & Mecodium \\
\hline jamesoni Hook. & Hymenophyllum & Hymenophyllum \\
\hline japonicum Miq. & Hymenophyllum & Hymenophyllum \\
\hline javanicum A. Spreng. & Hymenophyllum & Globosa \\
\hline johorense Holttum & Hymenophyllum & Hymenophyllum \\
\hline junghuhnii Bosch & Hymenophyllum & Globosa \\
\hline kaieteurum Jenman & Hymenophyllum & Sphaerocionium \\
\hline karstenianum Sturm & Hymenophyllum & Sphaerocionium \\
\hline kerianum Watts & Hymenophyllum & Hymenophyllum \\
\hline khasianum Baker & Hymenophyllum & Hymenophyllum \\
\hline klabatense H. Christ & Hymenophyllum & Hymenophyllum \\
\hline kohautianum C. Presl & Hymenophyllum & Mecodium \\
\hline krauseanum Phil. & Hymenophyllum & Myrmecostylum \\
\hline kuhnii C. Chr. & Hymenophyllum & Mecodium \\
\hline kurzii Prantl & Hymenophyllum & Hymenophyllum \\
\hline laciniosum $\mathrm{H}$. Christ & Hymenophyllum & Mecodium \\
\hline lamellatum Stolze & Hymenophyllum & Hymenophyllum \\
\hline laminatum Copel. & Hymenophyllum & Hymenophyllum \\
\hline lanatum Fée & Hymenophyllum & Sphaerocionium \\
\hline lanceolatum Hook. \& Arn. & Hymenophyllum & Sphaerocionium \\
\hline latifrons Bosch & Hymenophyllum & Sphaerocionium \\
\hline latilobum Bonap. & Hymenophyllum & Globosa \\
\hline ledermanni Brause & Hymenophyllum & Hymenophyllum \\
\hline lehmannii Hieron. & Hymenophyllum & Mecodium \\
\hline leptocarpum Copel. & Hymenophyllum & Hymenophyllum \\
\hline leptodictyon Müll.Berol. & Hymenophyllum & Globosa \\
\hline leratii Rosenst. & Hymenophyllum & Pleuromanes \\
\hline levingei C.B. Clarke & Hymenophyllum & Hymenophyllum \\
\hline limminghei Bosch & Hymenophyllum & Mecodium \\
\hline lindenii Hook. & Hymenophyllum & Sphaerocionium \\
\hline
\end{tabular}


Appendix. Continued.

\begin{tabular}{|c|c|c|}
\hline Basionym & Genus & Subgenus / Section \\
\hline \multicolumn{3}{|l|}{ Hymenophyllum (cont.) } \\
\hline lindigii Mett. & Hymenophyllum & Sphaerocionium \\
\hline lindsaeoides Baker & Sphenomeris & - \\
\hline lingganum Alderw. & Hymenophyllum & Hymenophyllum \\
\hline lobatoalatum Klotzsch & Hymenophyllum & Sphaerocionium \\
\hline lobatopapillosum Klotzsch ex Sadeb. & Hymenophyllum & Sphaerocionium \\
\hline lobbii $\mathrm{T}$. Moore ex Bosch & Hymenophyllum & Hymenophyllum \\
\hline longifolium Alderw. & Hymenophyllum & Globosa \\
\hline lophocarpum Colenso & Hymenophyllum & Myrmecostylum \\
\hline lyallii Hook.f. & Hymenophyllum & Sphaerocionium \\
\hline macroglossum Bosch & Hymenophyllum & Hymenophyllum \\
\hline macrosorum Alderw. & Hymenophyllum & Hymenophyllum \\
\hline macrothecum Fée & Hymenophyllum & Mecodium \\
\hline maderense Gibby \& Lovis & Hymenophyllum & Hymenophyllum \\
\hline malaccense Gand. & Hymenophyllum & Globosa \\
\hline mannianum Mett. ex Kuhn & Hymenophyllum & Hymenophyllum \\
\hline marginatum Hook. \& Grev. & Hymenophyllum & Hymenophyllum \\
\hline marlothii Brause & Hymenophyllum & Sphaerocionium \\
\hline martii Sturm & Hymenophyllum & Globosa \\
\hline martinicense Bosch & Hymenophyllum & Mecodium \\
\hline matthewsii Bosch & Hymenophyllum & Mecodium \\
\hline maxonii $\mathrm{H}$. Christ ex C.V. Morton & Hymenophyllum & Sphaerocionium \\
\hline mazei E. Fourn. ex H. Christ & Hymenophyllum & Mecodium \\
\hline megachilum C. Presl & Hymenophyllum & Hymenophyllum \\
\hline megalocarpum Colenso & Hymenophyllum & Globosa \\
\hline melanocheilos Colenso & Hymenophyllum & Hymenophyllum \\
\hline mentitum Gand. & Hymenophyllum & Globosa \\
\hline menziesii C. Presl & Hymenophyllum & Hymenophyllum \\
\hline merrillii H. Christ & Hymenophyllum & Hymenophyllum \\
\hline mettenii Bosch & Hymenophyllum & Hymenophyllum \\
\hline meyeri C. Presl & Hymenophyllum & Hymenophyllum \\
\hline micans $\mathrm{H}$. Christ & Hymenophyllum & Sphaerocionium \\
\hline micranthum Bosch & Hymenophyllum & Globosa \\
\hline microcarpon Fée & Hymenophyllum & Sphaerocionium \\
\hline microcarpum Desv. & Hymenophyllum & Sphaerocionium \\
\hline microphyllum Mett. & Hymenophyllum & Mecodium \\
\hline microsorum Bosch & Hymenophyllum & Mecodium \\
\hline millefolium Schltdl. \& Cham. & Hymenophyllum & Mecodium \\
\hline minimum A. Rich. & Hymenophyllum & Hymenophyllum \\
\hline minutidenticulatum Ching \& P.C. Chiu & Hymenophyllum & Hymenophyllum \\
\hline mirificum C.V. Morton & Hymenophyllum & Hymenophyllum \\
\hline mnioides Baker & Hymenophyllum & Mecodium \\
\hline modestum Bosch & Hymenophyllum & Globosa \\
\hline molle C.V. Morton & Hymenophyllum & Sphaerocionium \\
\hline montanum Kirk & Hymenophyllum & Globosa \\
\hline moorei Baker & Hymenophyllum & Hymenophyllum \\
\hline moritziaum Sturm & Hymenophyllum & Sphaerocionium \\
\hline mortonianum Lellinger & Hymenophyllum & Sphaerocionium \\
\hline multialatum C.V. Morton & Hymenophyllum & Sphaerocionium \\
\hline multiflorum Rosenst. & Hymenophyllum & Mecodium \\
\hline myriocarpum Hook. & Hymenophyllum & Mecodium \\
\hline nahuelhuapiense Diem \& J.S. Licht. & Hymenophyllum & Hymenophyllum \\
\hline nanostellatum Lellinger & Hymenophyllum & Sphaerocionium \\
\hline
\end{tabular}


Appendix. Continued.

\begin{tabular}{|c|c|c|}
\hline Basionym & Genus & Subgenus / Section \\
\hline \multicolumn{3}{|l|}{ Hymenophyllum (cont.) } \\
\hline nanum Sodiro & Hymenophyllum & Hymenophyllum \\
\hline natalense Bosch & Hymenophyllum & Mecodium \\
\hline neozelandicum Gand. & Hymenophyllum & Globosa \\
\hline nephrophyllum Ebihara \& K. Iwats. & Hymenophyllum & Cardiomanes \\
\hline nigrescens Liebm. & Hymenophyllum & Mecodium \\
\hline nigricans Colla & Hymenophyllum & Myrmecostylum \\
\hline nitens $\mathrm{R} . \mathrm{Br}$. & Hymenophyllum & Pleuromanes \\
\hline nitiduloides Copel. & Hymenophyllum & Mecodium \\
\hline notabile Fée & Hymenophyllum & Sphaerocionium \\
\hline nutantifolium Alderw. & Hymenophyllum & Hymenophyllum \\
\hline obtusum Hook. \& Arn. & Hymenophyllum & Sphaerocionium \\
\hline odontophyllum Copel. & Hymenophyllum & Hymenophyllum \\
\hline okadai Masam. & Hymenophyllum & Hymenophyllum \\
\hline oligocarpum Colenso & Hymenophyllum & Hymenophyllum \\
\hline oligosorum Makino & Hymenophyllum & Hymenophyllum \\
\hline omeiense H. Christ & Hymenophyllum & Hymenophyllum \\
\hline ooides F. Muell. \& Baker & Hymenophyllum & Mecodium \\
\hline opacum Copel. & Hymenophyllum & Globosa \\
\hline orbignianum Bosch & Hymenophyllum & Sphaerocionium \\
\hline organense Hook. & Hymenophyllum & Sphaerocionium \\
\hline osmundoides Bosch & Hymenophyllum & Mecodium \\
\hline ovatum Copel. & Hymenophyllum & Hymenophyllum \\
\hline oxyodon Baker & Hymenophyllum & Hymenophyllum \\
\hline pachydermicum Ces. & Hymenophyllum & Hymenophyllum \\
\hline palmatum Bosch & Hymenophyllum & Hymenophyllum \\
\hline palmense Rosenst. & Hymenophyllum & Sphaerocionium \\
\hline paniculiflorum C. Presl & Hymenophyllum & Mecodium \\
\hline paniense Ebihara \& K. Iwats. & Hymenophyllum & Myrmecostylum \\
\hline pannosum $\mathrm{H}$. Christ & Hymenophyllum & Sphaerocionium \\
\hline pantotactum Alderw. & Hymenophyllum & Globosa \\
\hline parallelocarpum Hayata & Hymenophyllum & Mecodium \\
\hline parvifolium Baker & Crepidomanes & Crepidomanes/Crepidomanes \\
\hline parvulum C. Chr. & Hymenophyllum & Mecodium \\
\hline pastoensis Hook. & Hymenophyllum & Sphaerocionium \\
\hline patagonicum Gand. & Hymenophyllum & Globosa \\
\hline paucicarpum Jenman & Hymenophyllum & Mecodium \\
\hline pectinatum Cav. & Hymenophyllum & Hymenophyllum \\
\hline pedicellatum Kunze ex Klotzsch & Hymenophyllum & Hymenophyllum \\
\hline pedicularifolium Ces. & Hymenophyllum & Hymenophyllum \\
\hline penangianum C.G. Matthew \& H. Christ & Hymenophyllum & Hymenophyllum \\
\hline pendulum Bory & Hymenophyllum & Sphaerocionium \\
\hline perfissum Copel. & Hymenophyllum & Hymenophyllum \\
\hline perparvulum Alderw. & Hymenophyllum & Hymenophyllum \\
\hline perrieri Tardieu & Hymenophyllum & Hymenophyllum \\
\hline peruvianum Hook. \& Grev. & Hymenophyllum & Hymenophyllum \\
\hline physocarpum $\mathrm{H}$. Christ & Hymenophyllum & Globosa \\
\hline piliferum C. Chr. & Hymenophyllum & Hymenophyllum \\
\hline pilosissimum C. Chr. & Hymenophyllum & Sphaerocionium \\
\hline pilosum Alderw. & Hymenophyllum & Hymenophyllum \\
\hline platylobum Bosch & Hymenophyllum & Sphaerocionium \\
\hline pleiocarpum Alderw. & Hymenophyllum & Globosa \\
\hline plicatum Kaulf. & Hymenophyllum & Myrmecostylum \\
\hline
\end{tabular}


Appendix. Continued.

\begin{tabular}{|c|c|c|}
\hline Basionym & Genus & Subgenus / Section \\
\hline \multicolumn{3}{|l|}{ Hymenophyllum (cont.) } \\
\hline plumieri Hook. \& Grev. & Hymenophyllum & Sphaerocionium \\
\hline plumosum Kaulf. & Hymenophyllum & Sphaerocionium \\
\hline podocarpon Fée & Hymenophyllum & Hymenophyllum \\
\hline poeppigianum C. Presl & Hymenophyllum & Mecodium \\
\hline poilanei Tardieu \& C. Chr. & Hymenophyllum & Hymenophyllum \\
\hline pollenianum Rosenst. & Hymenophyllum & Mecodium \\
\hline polyanthum Hook. & Callistopteris & - \\
\hline polycarpum Mett. ex Kuhn & Hymenophyllum & Mecodium \\
\hline polychilum Colenso & Hymenophyllum & Globosa \\
\hline polyodon Baker & Hymenophyllum & Hymenophyllum \\
\hline poolii Baker & Hymenophyllum & Sphaerocionium \\
\hline portoricense Kuhn & Hymenophyllum & Sphaerocionium \\
\hline praetervisum $\mathrm{H}$. Christ & Hymenophyllum & Hymenophyllum \\
\hline prionema Kunze ex Sturm & Hymenophyllum & Sphaerocionium \\
\hline procerum Bosch & Hymenophyllum & Sphaerocionium \\
\hline proctoris C. Sánchez & Hymenophyllum & Sphaerocionium \\
\hline producens Fée & Hymenophyllum & Sphaerocionium \\
\hline productoides J.W. Moore & Hymenophyllum & Globosa \\
\hline productum Kunze & Hymenophyllum & Globosa \\
\hline protrusum Hook. & Hymenophyllum & Mecodium \\
\hline pseudotunbridgense Watts & Hymenophyllum & Hymenophyllum \\
\hline pteropodum Bosch & Hymenophyllum & Sphaerocionium \\
\hline puellum Ces. & Abrodictyum? & Abrodictyum? \\
\hline pulchellum Schltdl. \& Cham. & Hymenophyllum & Sphaerocionium \\
\hline pulcherrimum Colenso & Hymenophyllum & Fuciformia \\
\hline pulchrum Copel. & Hymenophyllum & Hymenophyllum \\
\hline pumilio Rosenst. & Hymenophyllum & Hymenophyllum \\
\hline pumilum C. Moore & Hymenophyllum & Hymenophyllum \\
\hline punctisorum Rosenst. & Hymenophyllum & Mecodium \\
\hline pusillum Schott ex Sturm & Hymenophyllum & Mecodium \\
\hline pycnocarpum Bosch & Hymenophyllum & Mecodium \\
\hline pygmaeum Colenso & Hymenophyllum & Hymenophyllum \\
\hline pyramidatum Desv. & Hymenophyllum & Sphaerocionium \\
\hline pyriforme Bosch & Hymenophyllum & Hymenophyllum \\
\hline quadrifidum Phil. & Hymenophyllum & Myrmecostylum \\
\hline quetrihuense Diem \& J.S. Licht. & Hymenophyllum & Hymenophyllum \\
\hline raapii Gand. & Hymenophyllum & Globosa \\
\hline raddianum Müll.Berol. & Hymenophyllum & Sphaerocionium \\
\hline ramosii Copel. & Hymenophyllum & Hymenophyllum \\
\hline ramosissimum Ham. ex D. Don & Sphenomeris & - \\
\hline rarum $\mathrm{R} . \mathrm{Br}$. & Hymenophyllum & Mecodium \\
\hline recurvum Gaudich. & Hymenophyllum & Mecodium \\
\hline reductum Copel. & Hymenophyllum & Hymenophyllum \\
\hline refrondescens Sodiro & Hymenophyllum & Sphaerocionium \\
\hline reinwardtii Bosch & Hymenophyllum & Globosa \\
\hline remotipinna Bonap. & Hymenophyllum & Mecodium \\
\hline remotum Bosch & Hymenophyllum & Sphaerocionium \\
\hline reniforme Hook. & Hymenophyllum & Mecodium \\
\hline repens Dulac & Hymenophyllum & Hymenophyllum \\
\hline retusilobum Hayata & Hymenophyllum & Globosa \\
\hline revolutum Colenso & Hymenophyllum & Hymenophyllum \\
\hline ricciaefolium Bory ex Willd. & Hymenophyllum & Hymenophyllum \\
\hline
\end{tabular}


Appendix. Continued.

\begin{tabular}{|c|c|c|}
\hline Basionym & Genus & Subgenus / Sectic \\
\hline \multicolumn{3}{|l|}{ Hymenophyllum (cont.) } \\
\hline rimbachii Sodiro & Hymenophyllum & Mecodium \\
\hline ringens $\mathrm{H}$. Christ & Hymenophyllum & Hymenophyllum \\
\hline riukiuense $\mathrm{H}$. Christ & Hymenophyllum & Globosa \\
\hline rolandi-principis Rosenst. & Hymenophyllum & Hymenophyllum \\
\hline roraimense $\mathrm{C} . \mathrm{V}$. Morton & Hymenophyllum & Sphaerocionium \\
\hline rosenstockii Brause & Hymenophyllum & Hymenophyllum \\
\hline rubellum Rosenst. & Hymenophyllum & Hymenophyllum \\
\hline rufescens Kirk & Hymenophyllum & Pleuromanes \\
\hline rufifolium Alderw. & Hymenophyllum & Hymenophyllum \\
\hline rufifrons Alderw. & Hymenophyllum & Hymenophyllum \\
\hline rufofibrillosum Ching \& Z.Y. Liu & Hymenophyllum & Hymenophyllum \\
\hline rufum Fée & Hymenophyllum & Sphaerocionium \\
\hline rugosum C. Chr. \& Skottsb. & Hymenophyllum & Hymenophyllum \\
\hline rupestre Raddi & Vandenboschia & Lacosteopsis \\
\hline sabinifolium Baker & Hymenophyllum & Hymenophyllum \\
\hline saenzianum L.D. Gómez & Hymenophyllum & Sphaerocionium \\
\hline salakense Racib. & Hymenophyllum & Globosa \\
\hline samoense Baker & Hymenophyllum & Globosa \\
\hline sampaioanum Brade \& Rosenst. & Hymenophyllum & Sphaerocionium \\
\hline scabrum A. Rich. & Hymenophyllum & Myrmecostylum \\
\hline schomburghii C. Presl & Hymenophyllum & Mecodium \\
\hline secundum Hook. \& Grev. & Hymenophyllum & Hymenophyllum \\
\hline semibivalve Hook. \& Grev. & Hymenophyllum & Mecodium \\
\hline semifissum Copel. & Hymenophyllum & Hymenophyllum \\
\hline semiglabrum Rosenst. & Hymenophyllum & Sphaerocionium \\
\hline semiteres Colla & Hymenophyllum & Fuciformia \\
\hline serra C. Presl & Hymenophyllum & Hymenophyllum \\
\hline seselifolium C. Presl & Hymenophyllum & Myrmecostylum \\
\hline shirleyanum Domin & Hymenophyllum & Globosa \\
\hline siliquosum $\mathrm{H}$. Christ & Hymenophyllum & Mecodium \\
\hline silvaticum C.V. Morton & Hymenophyllum & Sphaerocionium \\
\hline silveirae $\mathrm{H}$. Christ & Hymenophyllum & Sphaerocionium \\
\hline simonsianum Hook. & Hymenophyllum & Hymenophyllum \\
\hline simplex C.V. Morton & Hymenophyllum & Sphaerocionium \\
\hline skottsbergii C. Chr. & Hymenophyllum & Mecodium \\
\hline smithii Hook. & Hymenophyllum & Hymenophyllum \\
\hline sodiroi C. Chr. & Hymenophyllum & Hymenophyllum \\
\hline spathulatum Colenso & Hymenophyllum & Hymenophyllum \\
\hline speciosum Bosch & Hymenophyllum & Sphaerocionium \\
\hline spectabile Mett. ex Kuhn & Hymenophyllum & Sphaerocionium \\
\hline sphaerocarpum Bosch & Hymenophyllum & Mecodium \\
\hline spicatum $\mathrm{H}$. Christ & Hymenophyllum & Hymenophyllum \\
\hline spinosum Ching & Hymenophyllum & Hymenophyllum \\
\hline spinulosum Kunth & Hymenophyllum & Hymenophyllum \\
\hline splendidum Bosch & Hymenophyllum & Sphaerocionium \\
\hline sprucei Baker & Hymenophyllum & Sphaerocionium? \\
\hline steerei C. Chr. & Hymenophyllum & Globosa \\
\hline streptophyllum E. Fourn. & Hymenophyllum & Globosa \\
\hline sturmii Bosch & Hymenophyllum & Mecodium \\
\hline subdeltoideum $\mathrm{H}$. Christ & Hymenophyllum & Mecodium \\
\hline subdemissum $\mathrm{H}$. Christ & Hymenophyllum & Mecodium \\
\hline subdimidiatum Rosenst. & Hymenophyllum & Hymenophyllum \\
\hline
\end{tabular}


Appendix. Continued.

\begin{tabular}{|c|c|c|}
\hline Basionym & Genus & Subgenus / Sectic \\
\hline \multicolumn{3}{|l|}{ Hymenophyllum (cont.) } \\
\hline subfirmum Alderw. & Hymenophyllum & Hymenophyllum \\
\hline subflabellatum Ces. & Hymenophyllum & Hymenophyllum \\
\hline subobtusum Rosenst. & Hymenophyllum & Sphaerocionium \\
\hline subrigidum $\mathrm{H}$. Christ & Hymenophyllum & Sphaerocionium \\
\hline subrotundum Alderw. & Hymenophyllum & Hymenophyllum \\
\hline subtilissimum Kunze & Hymenophyllum & Sphaerocionium \\
\hline superbum C.V. Morton & Hymenophyllum & Sphaerocionium \\
\hline suprapaleaceum Ching & Hymenophyllum & Hymenophyllum \\
\hline surinamense Bosch & Hymenophyllum & Sphaerocionium \\
\hline tablaziense $\mathrm{H}$. Christ & Hymenophyllum & Mecodium \\
\hline tabulare Bosch & Hymenophyllum & Mecodium \\
\hline taliabense Alderw. & Hymenophyllum & Hymenophyllum \\
\hline tarapotense Stolze & Hymenophyllum & Sphaerocionium \\
\hline tasmannicum Bosch & Hymenophyllum & Globosa \\
\hline tayloriae Farrar \& Raine & Hymenophyllum & Sphaerocionium \\
\hline tenellum D. Don & Hymenophyllum & Mecodium \\
\hline tenerrimum Bosch & Hymenophyllum & Sphaerocionium \\
\hline tenerum Bosch & Hymenophyllum & Mecodium \\
\hline terminale Bosch & Hymenophyllum & Sphaerocionium \\
\hline thomassetii C.H. Wright & Hymenophyllum & Hymenophyllum \\
\hline thuidium Harr. & Hymenophyllum & Globosa \\
\hline thunbergii Eckl. ex C. Presl & Hymenophyllum & Mecodium \\
\hline todjambuense Kjellb. & Hymenophyllum & Globosa \\
\hline tomentosum Kunze & Hymenophyllum & Sphaerocionium \\
\hline torquescens Bosch & Hymenophyllum & Hymenophyllum \\
\hline torricellianum Alderw. & Hymenophyllum & Hymenophyllum \\
\hline tortuosum Hook. \& Grev. & Hymenophyllum & Myrmecostylum \\
\hline trapezoidale Liebm. & Hymenophyllum & Sphaerocionium \\
\hline treubii Racib. & Hymenophyllum & Globosa \\
\hline trianae Hieron. & Hymenophyllum & Mecodium \\
\hline triangulare Baker & Hymenophyllum & Hymenophyllum \\
\hline trichocaulon Phil. & Hymenophyllum & Hymenophyllum \\
\hline trichomanoides Bosch & Hymenophyllum & Mecodium \\
\hline trichophyllum Kunth & Hymenophyllum & Sphaerocionium \\
\hline trifidum Hook. \& Grev. & Hymenophyllum & Sphaerocionium \\
\hline truncatum Colenso & Hymenophyllum & Hymenophyllum \\
\hline turquinense C. Sánchez & Hymenophyllum & Sphaerocionium \\
\hline ulei H. Christ \& Giesenh. & Hymenophyllum & Sphaerocionium \\
\hline umbratile Diem \& J.S. Licht. & Hymenophyllum & Hymenophyllum \\
\hline uncinatum Sim & Hymenophyllum & Hymenophyllum \\
\hline unilaterale Bory ex Willd. & Hymenophyllum & Hymenophyllum \\
\hline urbanii Brause & Hymenophyllum & Sphaerocionium \\
\hline urofrons Ching \& C.F. Zhang & Hymenophyllum & Hymenophyllum \\
\hline vacillans $\mathrm{H}$. Christ & Hymenophyllum & Hymenophyllum? \\
\hline valvatum Hook. \& Grev. & Hymenophyllum & Sphaerocionium \\
\hline venustum Desv. & Hymenophyllum & Sphaerocionium \\
\hline verecundum C.V. Morton & Hymenophyllum & Sphaerocionium \\
\hline veronicoides $\mathrm{C}$. Chr. & Hymenophyllum & Mecodium \\
\hline viguieri Tardieu & Hymenophyllum & Sphaerocionium \\
\hline villosum Colenso & Hymenophyllum & Myrmecostylum \\
\hline vincentinum Baker & Hymenophyllum & Mecodium \\
\hline viride Rosenst. ex Copel. & Hymenophyllum & Hymenophyllum \\
\hline
\end{tabular}


Appendix. Continued.

\begin{tabular}{|c|c|c|}
\hline Basionym & Genus & Subgenus / Section \\
\hline \multicolumn{3}{|l|}{ Hymenophyllum (cont.) } \\
\hline viridissimum Fée & Hymenophyllum & Mecodium \\
\hline vittatum Copel. & Hymenophyllum & Hymenophyllum \\
\hline walleri Maiden \& Betche & Hymenophyllum & Hymenophyllum \\
\hline wercklei $\mathrm{H}$. Christ & Hymenophyllum & Sphaerocionium \\
\hline whangshanense Ching \& P.C. Chiu & Hymenophyllum & Hymenophyllum \\
\hline whitei Goy & Hymenophyllum & Mecodium \\
\hline wilsonii Hook. & Hymenophyllum & Hymenophyllum \\
\hline wrightii Bosch & Hymenophyllum & Mecodium \\
\hline zeelandicum Bosch & Hymenophyllum & Hymenophyllum \\
\hline zeyheri Bosch & Hymenophyllum & Mecodium \\
\hline zollingerianum Kunze & Hymenophyllum & Hymenophyllum \\
\hline \multicolumn{3}{|l|}{ Hymenostachys } \\
\hline diversifrons Bory & Trichomanes & Feea \\
\hline elegans C. Presl & Trichomanes & Feea \\
\hline \multicolumn{3}{|l|}{ Leptocionium } \\
\hline barbatum Bosch & Hymenophyllum & Hymenophyllum \\
\hline dicranotrichum C. Presl & Hymenophyllum & Hymenophyllum \\
\hline edentulum Bosch & Hymenophyllum & Hymenophyllum \\
\hline flaccidum Bosch & Hymenophyllum & Hymenophyllum \\
\hline sororium $\mathrm{C}$. Presl & Hymenophyllum & Diploophyllum \\
\hline violaceum Meyen ex Bosch & Hymenophyllum & Hymenophyllum \\
\hline \multicolumn{3}{|l|}{ Macroglena } \\
\hline brassii Croxall & Abrodictyum & Abrodictyum \\
\hline kalimantanensis K. Iwats. \& M. Kato & Abrodictyum & Pachychaetum \\
\hline obtusa Copel. & Vandenboschia & Vandenboschia \\
\hline toppingii O. Deg. \& I. Deg. & Callistopteris & - \\
\hline truncata Copel. & Abrodictyum & Abrodictyum \\
\hline \multicolumn{3}{|l|}{ Mecodium } \\
\hline acrocarpum $\mathrm{H}$. Christ ex Ching & Hymenophyllum & Mecodium \\
\hline archboldii Copel. & Hymenophyllum & Mecodium \\
\hline contiguum D.A. Sm. & Hymenophyllum & Mecodium \\
\hline diversilabium Copel. & Hymenophyllum & Mecodium \\
\hline edanoi Copel. & Hymenophyllum & Globosa \\
\hline gongboense Ching & Hymenophyllum & Mecodium? \\
\hline hainanense Ching & Hymenophyllum & Mecodium \\
\hline jinfoshanense Ching \& Z.Y. Liu & Hymenophyllum & Mecodium \\
\hline kansuense Ching \& Y.P. Hsu & Hymenophyllum & Mecodium \\
\hline likiangense Ching \& P.C. Chiu & Hymenophyllum & Mecodium \\
\hline lineatum Ching \& P.C. Chiu & Hymenophyllum & Globosa \\
\hline lofoushanense Ching \& P.C. Chiu & Hymenophyllum & Mecodium? \\
\hline longissimum Ching \& P.C. Chiu & Hymenophyllum & Mecodium? \\
\hline lushanense Ching \& P.C. Chiu & Hymenophyllum & Mecodium \\
\hline mexiae Copel. & Hymenophyllum & Mecodium \\
\hline mikawanum Seriz. & Hymenophyllum & Mecodium \\
\hline ovalifolium Ching \& P.C. Chiu & Hymenophyllum & Mecodium \\
\hline paramnioides H.G. Zhou \& W.M. Chu & Hymenophyllum & Mecodium \\
\hline propinquum Ching \& P.C. Chiu & Hymenophyllum & Globosa \\
\hline stenochladum Ching \& P.C. Chiu & Hymenophyllum & Globosa \\
\hline szechuanense Ching \& P.C. Chiu & Hymenophyllum & Mecodium \\
\hline taiwanense Tagawa & Hymenophyllum & Globosa \\
\hline tenellum Parris & Hymenophyllum & Mecodium \\
\hline tenuifrons Ching & Hymenophyllum & Globosa \\
\hline
\end{tabular}


Appendix. Continued.

\begin{tabular}{|c|c|c|}
\hline Basionym & Genus & Subgenus / Section \\
\hline \multicolumn{3}{|l|}{ Mecodium (cont.) } \\
\hline wangii Ching \& P.C. Chiu & Hymenophyllum & Mecodium \\
\hline wenhsienense Ching \& Y.P. Hsu & Hymenophyllum & Mecodium \\
\hline \multicolumn{3}{|l|}{ Meringium } \\
\hline archboldii Copel. & Hymenophyllum & Hymenophyllum \\
\hline bartlettii Copel. & Hymenophyllum & Hymenophyllum \\
\hline blumeanum C. Presl & Hymenophyllum & Mecodium \\
\hline latifolium Copel. & Hymenophyllum & Hymenophyllum \\
\hline laxum Copel. & Hymenophyllum & Hymenophyllum \\
\hline melanosorum Copel. & Hymenophyllum & Hymenophyllum \\
\hline meyenianum C. Presl & Hymenophyllum & Hymenophyllum \\
\hline \multicolumn{3}{|l|}{ Microgonium } \\
\hline benlii Pic.Serm. & Didymoglossum & Microgonium \\
\hline berteroanum C. Presl & Didymoglossum & Microgonium \\
\hline bimarginatum Bosch & Didymoglossum & Microgonium \\
\hline falsinervulosum M. Nishida & Didymoglossum & Microgonium \\
\hline minutifolium Tagawa \& K. Iwats. & Crepidomanes & Crepidomanes/Crepidomanes \\
\hline omphalodes Vieill. ex E. Fourn. & Didymoglossum & Didymoglossum \\
\hline \multicolumn{3}{|l|}{ Microtrichomanes } \\
\hline zamboanganum Copel. & Hymenophyllum & Sphaerocionium \\
\hline \multicolumn{3}{|l|}{ Neuromanes } \\
\hline abruptum Bosch & Trichomanes & Trichomanes \\
\hline immersum Bosch & Trichomanes & Trichomanes \\
\hline kaulfussii Bosch & Trichomanes & Trichomanes \\
\hline \multicolumn{3}{|l|}{ Neurophyllum } \\
\hline hedwigianum Fée & Trichomanes & Trichomanes \\
\hline hostmannianum Klotzsch & Trichomanes & Trichomanes \\
\hline thecaphyllum Fée & Trichomanes & Trichomanes \\
\hline \multicolumn{3}{|l|}{ Pleuromanes } \\
\hline retusum Copel. & Hymenophyllum & Pleuromanes \\
\hline \multicolumn{3}{|l|}{ Ragatelus } \\
\hline eriophorus C. Presl & Trichomanes & Trichomanes \\
\hline \multicolumn{3}{|l|}{ Selenodesmium } \\
\hline recurvum Ching \& P.C. Chiu & Abrodictyum & Pachychaetum \\
\hline \multicolumn{3}{|l|}{ Sphaerocionium } \\
\hline aureum C. Presl & Hymenophyllum & Sphaerocionium \\
\hline breutelli C. Presl & Hymenophyllum & Mecodium \\
\hline commutatum C. Presl & Hymenophyllum & Sphaerocionium \\
\hline diversilobum C. Presl & Hymenophyllum & Sphaerocionium \\
\hline glanduliferum C. Presl & Hymenophyllum & Myrmecostylum \\
\hline grevilleanum C. Presl & Hymenophyllum & Sphaerocionium \\
\hline macrocarpum C. Presl & Hymenophyllum & Globosa \\
\hline nigricans $\mathrm{C}$. Presl & Hymenophyllum & Mecodium \\
\hline productum C. Presl & Hymenophyllum & Globosa \\
\hline ruizianum Klotzsch & Hymenophyllum & Sphaerocionium \\
\hline schiedeanum C. Presl & Hymenophyllum & Sphaerocionium \\
\hline sieberi C. Presl & Hymenophyllum & Sphaerocionium \\
\hline$\times$ tucuchense Jermy \& T.G. Walker & Hymenophyllum & Sphaerocionium \\
\hline vestitum $\mathrm{C}$. Presl & Hymenophyllum & Sphaerocionium \\
\hline \multicolumn{3}{|l|}{ Trichomanes } \\
\hline abrotanifolium Bosch & Vandenboschia? & $?$ \\
\hline abruptum Fée ex H. Christ & Trichomanes & Trichomanes \\
\hline accedens C. Presl & Trichomanes & Trichomanes \\
\hline
\end{tabular}


Appendix. Continued.

\begin{tabular}{|c|c|c|}
\hline Basionym & Genus & Subgenus / Section \\
\hline \multicolumn{3}{|l|}{ Trichomanes (cont.) } \\
\hline achilleaefolium Bory ex Willd. & Abrodictyum & Pachychaetum \\
\hline acranthum $\mathrm{H}$. Itô & Cephalomanes & - \\
\hline acrocarpon Fée & Didymoglossum & Didymoglossum \\
\hline acrosorum Copel. & Cephalomanes & - \\
\hline acutilobum Ching & Crepidomanes & Crepidomanes/Crepidomanes \\
\hline acuto-obtusum Hayata & Crepidomanes & Crepidomanes/Crepidomanes \\
\hline acutum C. Presl & Hymenophyllum & Pleuromanes \\
\hline adianthinum Bory & Didymoglossum & Microgonium \\
\hline adiantoides $\mathrm{L}$. & Asplenium & - \\
\hline adscendens Kunze & Trichomanes? & $?$ \\
\hline aerugineum Bosch & Didymoglossum & Microgonium \\
\hline aeruginosum Poir. & Hymenophyllum & Sphaerocionium \\
\hline aethiopicum Burm.f. & Asplenium & - \\
\hline africanum $\mathrm{H}$. Christ & Crepidomanes & Crepidomanes/Crepidomanes \\
\hline alagense $\mathrm{H}$. Christ & Crepidomanes & Crepidomanes/Gonocormus \\
\hline alatum $\mathrm{Sw}$. & Trichomanes & Trichomanes \\
\hline album Blume & Hymenophyllum & Pleuromanes \\
\hline alternans Carruth. ex Seem. & Polyphlebium & - \\
\hline amabile Nakai & Vandenboschia & Vandenboschia \\
\hline amazonicum $\mathrm{H}$. Christ & Trichomanes & Trichomanes \\
\hline ambiguum Mart. & Polyphlebium & - \\
\hline ambongoense Bonap. & Crepidomanes & Crepidomanes/Gonocormus \\
\hline anadromum Rosenst. & Trichomanes & Trichomanes \\
\hline anceps Hook. & Trichomanes & Davalliopsis \\
\hline andrewsii Newman & Vandenboschia & Vandenboschia \\
\hline angustatum Carmich. & Polyphlebium & - \\
\hline angustilaciniatum Bonap. & Crepidomanes & Crepidomanes/Gonocormus \\
\hline angustimarginatum Bonap. & Abrodictyum & Pachychaetum \\
\hline angustissimum $\mathrm{C}$. Presl & Polyphlebium & - \\
\hline ankersii C. Parker ex Hook. \& Grev. & Trichomanes & Lacostea \\
\hline anomalum Maxon \& C.V. Morton & Trichomanes & Trichomanes \\
\hline antillarum Bosch & Vandenboschia & Vandenboschia \\
\hline aphlebioides $\mathrm{H}$. Christ & Crepidomanes & Nesopteris \\
\hline apicilare E. Fourn. & Crepidomanes & Crepidomanes/Crepidium \\
\hline apiifolium C. Presl & Callistopteris & - \\
\hline apodum Hook. \& Grev. & Didymoglossum & Didymoglossum \\
\hline arbuscula Desv. & Trichomanes & Trichomanes \\
\hline armstrongii Baker & Hymenophyllum & Hymenophyllum \\
\hline asae-grayi Bosch & Abrodictyum & Abrodictyum \\
\hline asnykii Racib. & Crepidomanes & Crepidomanes/Crepidomanes \\
\hline asplenioides Sw. & Hymenophyllum & Hymenoglossum \\
\hline assimile Mett. ex Kuhn & Crepidomanes & Crepidomanes/Gonocormus \\
\hline astylum Kaulf. & Trichomanes & Trichomanes \\
\hline attenuatum Hook. & Trichomanes & Trichomanes \\
\hline auratum Fée & Trichomanes & Trichomanes \\
\hline aureovestitum Proctor & Trichomanes & Trichomanes \\
\hline aureum Bosch & Polyphlebium & - \\
\hline auriculatum Blume & Vandenboschia & Lacosteopsis \\
\hline axillare Sodiro & Polyphlebium? & - \\
\hline badium E. Fourn. & Trichomanes & Trichomanes \\
\hline baileyanum Watts & Didymoglossum & Microgonium \\
\hline ballardianum Alston & Didymoglossum & Microgonium \\
\hline
\end{tabular}


Appendix. Continued.

\begin{tabular}{|c|c|c|}
\hline Basionym & Genus & Subgenus / Section \\
\hline \multicolumn{3}{|l|}{ Trichomanes (cont.) } \\
\hline bancroftii Hook. \& Grev. & Trichomanes & Trichomanes \\
\hline barklianum Baker & Didymoglossum & Didymoglossum \\
\hline barnardianum F.M. Bailey & Crepidomanes & Crepidomanes/Crepidomanes \\
\hline batrachoglossum Copel. & Abrodictyum? & ? \\
\hline bauerianum Endl. & Callistopteris & - \\
\hline beccarianum Ces. & Didymoglossum & Didymoglossum \\
\hline beckeri Krause ex Phil. & Hymenophyllum & Myrmecostylum \\
\hline belangeri Bory & Vandenboschia & Lacosteopsis \\
\hline bicorne Hook. & Trichomanes & Trichomanes \\
\hline bifidum Vent. ex Willd. & Abrodictyum & Pachychaetum \\
\hline bifolium Blume & Crepidomanes & Crepidomanes/Gonocormus \\
\hline bilabiatum Nees \& Blume & Crepidomanes & Crepidomanes/Crepidomanes \\
\hline bilobatum Alderw. & Crepidomanes & Crepidomanes/Crepidomanes \\
\hline bipunctatum Poir. & Crepidomanes & Crepidomanes/Crepidomanes \\
\hline birmanicum Bedd. & Vandenboschia & Vandenboschia \\
\hline bissei C. Sánchez & Trichomanes & Trichomanes \\
\hline bivalve G. Forst. & Hymenophyllum & Hymenophyllum \\
\hline blepharistomum Copel. & Crepidomanes & Nesopteris \\
\hline blumei Hassk. & Hymenophyllum & Sphaerocionium \\
\hline bojeri Hook. \& Grev. & Didymoglossum & Microgonium \\
\hline bonapartei C. Chr. & Crepidomanes & Crepidomanes/Crepidomanes? \\
\hline bonincolum Nakai & Crepidomanes & Crepidomanes/Gonocormus \\
\hline boninense Koidz. & Crepidomanes & Crepidomanes/Crepidomanes \\
\hline borbonicum Bosch & Polyphlebium & - \\
\hline borneense Alderw. & Cephalomanes & - \\
\hline boryanum Kunze & Cephalomanes & - \\
\hline boschianum Sturm ex Bosch & Vandenboschia & Vandenboschia \\
\hline botryoides Kaulf. & Trichomanes & Feea \\
\hline bovini Bosch & Trichomanes & $?$ \\
\hline brachyblastos Mett. ex Kuhn & Vandenboschia & Vandenboschia \\
\hline brachypus Kunze & Trichomanes & Lacostea \\
\hline bradei $\mathrm{H}$. Christ & Polyphlebium & - \\
\hline brasiliense Desv. & Polyphlebium & - \\
\hline braunii Bosch & Hymenophyllum & Pleuromanes \\
\hline brevisetum $\mathrm{R} . \mathrm{Br}$. & Vandenboschia & Vandenboschia \\
\hline brooksii Copel. & Crepidomanes & Crepidomanes/Gonocormus \\
\hline bryoides Goldm. & $?$ & $?$ \\
\hline bucinatum Mickel \& Beitel & Didymoglossum & Didymoglossum \\
\hline caespifrons C. Chr. & Abrodictyum & Pachychaetum \\
\hline caliginum Lellinger & Trichomanes & Trichomanes \\
\hline caluffii C. Sánchez & Didymoglossum & $?$ \\
\hline calvescens Bosch & Hymenophyllum & Sphaerocionium \\
\hline campanulatum Roxb. & Crepidomanes? & $?$ \\
\hline canariensis $\mathrm{L}$. & Davallia & - \\
\hline capillaceum $\mathrm{L}$. & Polyphlebium & - \\
\hline capillatum Taschner & Crepidomanes & Crepidomanes/Crepidomanes \\
\hline carolianum Vareschi & Trichomanes & Lacostea \\
\hline cartilagineum Vieill. \& Pancher & Abrodictyum & Pachychaetum \\
\hline caruifolium Roxb. & Vandenboschia? & $?$ \\
\hline caudatum Brack. & Abrodictyum & Abrodictyum \\
\hline cavifolium Müll.Berol. & Polyphlebium & - \\
\hline cellulosum Klotzsch & Abrodictyum & Pachychaetum \\
\hline
\end{tabular}


Appendix. Continued.

\begin{tabular}{|c|c|c|}
\hline Basionym & Genus & Subgenus / Section \\
\hline \multicolumn{3}{|l|}{ Trichomanes (cont.) } \\
\hline chamaedrys Taton & Didymoglossum & Microgonium \\
\hline charophylloides Poir. & Davallia & - \\
\hline chevalieri $\mathrm{H}$. Christ & Crepidomanes & Crepidomanes/Crepidomanes \\
\hline chinensis L. & Sphenomeris & - \\
\hline christii Copel. & Crepidomanes & Crepidomanes/Crepidomanes \\
\hline ciliatum Sw. & Hymenophyllum & Sphaerocionium \\
\hline clarenceanum F. Ballard & Crepidomanes & Crepidomanes/Crepidomanes \\
\hline clathratum Tagawa & Abrodictyum & Abrodictyum \\
\hline clavatum $\mathrm{Sw}$. & Hymenophyllum & Mecodium \\
\hline cochinchinense Poir. & Lygodium & - \\
\hline $\operatorname{cocos}$ H. Christ & Polyphlebium & - \\
\hline cognatum $\mathrm{C}$. Presl & Trichomanes & Trichomanes \\
\hline colensoi Hook. & Polyphlebium & - \\
\hline collariatum Bosch & Vandenboschia & Vandenboschia \\
\hline commutatum Sturm & Trichomanes & Lacostea \\
\hline compactum Alderw. & Abrodictyum & Abrodictyum \\
\hline compressum Desv. & Abrodictyum & Pachychaetum \\
\hline concinnum Mett. ex Kuhn & Crepidomanes & Crepidomanes/Crepidium \\
\hline contiguum G. Forst. & Davallia & - \\
\hline corcovadense Bosch & Vandenboschia? & $?$ \\
\hline coriaceum Kunze & Trichomanes & Trichomanes \\
\hline cormophylum Kaulf. & Cyathea & - \\
\hline cornutum C. Chr. & Trichomanes? & $?$ \\
\hline corticola Hook. ex Bedd. & Hymenophyllum & Sphaerocionium \\
\hline craspedoneurum Copel. & Didymoglossum & Microgonium \\
\hline crassipilis Weath. & Trichomanes & Trichomanes \\
\hline crassum Copel. & Cephalomanes & - \\
\hline crenatum Bosch & Trichomanes & Trichomanes \\
\hline crinitum Sw. & Trichomanes & Trichomanes \\
\hline crispiforme Alston & Trichomanes & Trichomanes \\
\hline crispulum Bosch & Didymoglossum & Microgonium \\
\hline crispum $\mathrm{L}$. & Trichomanes & Trichomanes \\
\hline cristatum Kaulf. & Trichomanes & Trichomanes \\
\hline cultratum Baker & Didymoglossum & Didymoglossum \\
\hline cuneatum H. Christ & Hymenophyllum & Hymenophyllum? \\
\hline cuneiforme G. Forst. & Odontosoria & - \\
\hline cupressifolium Hayata & Crepidomanes & Crepidomanes/Crepidomanes \\
\hline cupressoides Desv. & Abrodictyum & Pachychaetum \\
\hline curranii Weath. & Trichomanes & Trichomanes \\
\hline curtii Rosenst. & Didymoglossum & Didymoglossum \\
\hline cuspidatum Willd. & Didymoglossum & Microgonium \\
\hline cyrtotheca Hillebr. & Vandenboschia & Vandenboschia \\
\hline cystoseiroides $\mathrm{H}$. Christ ex Tardieu \& C. Chr. & Vandenboschia & Vandenboschia \\
\hline dactylites Sodiro & Trichomanes & Trichomanes \\
\hline daguense Weath. & Trichomanes & Trichomanes \\
\hline daucoides C. Presl & Abrodictyum & Pachychaetum \\
\hline davallioides Gaudich. & Vandenboschia & Vandenboschia \\
\hline debile Bosch & Polyphlebium & - \\
\hline delicatum Bosch & Trichomanes & Trichomanes \\
\hline demissum G. Forst. & Hymenophyllum & Globosa \\
\hline densinervium Copel. & Cephalomanes & - \\
\hline dentatum Bosch & Abrodictyum & Pachychaetum \\
\hline
\end{tabular}


Appendix. Continued.

\begin{tabular}{|c|c|c|}
\hline Basionym & Genus & Subgenus / Section \\
\hline \multicolumn{3}{|l|}{ Trichomanes (cont.) } \\
\hline denticulatum Houtt. & Davallia & - \\
\hline depauperatum Bory & Crepidomanes & Crepidomanes/Crepidium \\
\hline diaphanum Kunth & Polyphlebium & - \\
\hline dichotomum Kunze & Hymenophyllum & Sphaerocionium \\
\hline diffusum Blume & Crepidomanes & Crepidomanes/Gonocormus \\
\hline digitatum $\mathrm{Sw}$. & Hymenophyllum & Sphaerocionium \\
\hline dilatatum G. Forst. & Hymenophyllum & Diploophyllum \\
\hline dimidiatum C. Pres1 & Vandenboschia & Lacosteopsis \\
\hline dimorphum Mett. ex Sadeb. & Trichomanes & Feea \\
\hline dissectum J. Sm. ex Hook. & Vandenboschia & Lacosteopsis \\
\hline divaricatum Poir. & Hymenophyllum & $?$ \\
\hline draytonianum Brack. & Crepidomanes & Crepidomanes/Crepidomanes \\
\hline dregei Bosch & Abrodictyum & Pachychaetum \\
\hline egleri P.G. Windisch & Trichomanes & Trichomanes \\
\hline ekmanii Wess.Boer & Didymoglossum & Microgonium \\
\hline elatum G. Forst. & Davallia & - \\
\hline elegans Rich. & Trichomanes & Davalliopsis \\
\hline elongatum A. Cunn. & Abrodictyum & Pachychaetum \\
\hline eminens C. Presl & Callistopteris & - \\
\hline endlicherianum C. Presl & Polyphlebium & - \\
\hline englerianum Brause & Abrodictyum & Pachychaetum \\
\hline epiphyllum G. Forst. & Davallia & - \\
\hline erectum Brack. & Polyphlebium & - \\
\hline ericoides Hedw. ex Hook. & Abrodictyum & $?$ \\
\hline erosum Willd. & Didymoglossum & Microgonium \\
\hline europaeum Sm. & Vandenboschia & Vandenboschia \\
\hline exaltatum Brack. & Callistopteris & - \\
\hline eximium Kunze ex Sturm & Polyphlebium & - \\
\hline exsectum Kunze & Polyphlebium & - \\
\hline extravagans Copel. & Abrodictyum & Pachychaetum \\
\hline fallax $\mathrm{H}$. Christ & Crepidomanes & Crepidomanes/Crepidomanes \\
\hline fargesii $\mathrm{H}$. Christ & Vandenboschia & Lacosteopsis? \\
\hline ferrugineum $\mathrm{E}$. Fourn. & Abrodictyum & Pachychaetum \\
\hline filiculoides H. Christ & Crepidomanes & Crepidomanes/Crepidium \\
\hline filiforme Sturm & Abrodictyum & Pachychaetum \\
\hline fimbriatum Backh. ex T. Moore & Trichomanes & Trichomanes \\
\hline firmulum C. Presl & Abrodictyum & Pachychaetum \\
\hline flabellatum Bory & Hymenophyllum & Hymenophyllum \\
\hline flabelliforme Hassk. & Hymenophyllum & Sphaerocionium \\
\hline flabellula d'Urv. & Hymenophyllum & Hymenophyllum \\
\hline flaccidum G. Forst. & Dennstaedtia & - \\
\hline flavofuscum Bosch & Abrodictyum & Abrodictyum \\
\hline floribundum Hook. \& Grev. & Trichomanes & Trichomanes \\
\hline foeniculaceum Bory ex Willd. & Abrodictyum & Pachychaetum \\
\hline foersteri Rosenst. & Cephalomanes & - \\
\hline fontanum Lindm. & Didymoglossum & Didymoglossum \\
\hline formosanum Y. Yabe & Crepidomanes & Crepidomanes/Crepidomanes \\
\hline fragile Hedw. & Hymenophyllum & Sphaerocionium \\
\hline francii $\mathrm{H}$. Christ & Hymenophyllum & Hymenophyllum ? \\
\hline frappieri Cordem. & Crepidomanes & Crepidomanes/Crepidomanes \\
\hline fraseri Jenman & Didymoglossum & Didymoglossum \\
\hline frondosum Fée & Vandenboschia & Lacosteopsis \\
\hline
\end{tabular}


Appendix. Continued.

\begin{tabular}{|c|c|c|}
\hline Basionym & Genus & Subgenus / Section \\
\hline \multicolumn{3}{|l|}{ Trichomanes (cont.) } \\
\hline fruticulosum Jenman & Didymoglossum & Didymoglossum \\
\hline fucoides Sw. & Hymenophyllum & Hymenophyllum \\
\hline fulgens C. Chr. & Didymoglossum & Didymoglossum \\
\hline fulvum Klotzsch & Polyphlebium & - \\
\hline furcatum Kaulf. ex Spreng. & Abrodictyum & Pachychaetum? \\
\hline fuscum Blume & Hymenophyllum & Hymenophyllum \\
\hline galeottii E. Fourn. & Trichomanes & Trichomanes \\
\hline gardneri Bosch & Trichomanes & Trichomanes \\
\hline gemmatum J. Sm. ex Baker & Abrodictyum & Pachychaetum \\
\hline gibberosum $\mathrm{G}$. Forst. & Asplenium & - \\
\hline giesenhagenii C. Chr. & Didymoglossum & Didymoglossum \\
\hline giganteum Bory ex Willd. & Vandenboschia & Vandenboschia \\
\hline glaucofuscum Hook. & Hymenophyllum & Pleuromanes \\
\hline godmanii Hook. & Didymoglossum & Microgonium \\
\hline goebelianum Giesenh. & Didymoglossum & Didymoglossum \\
\hline goetzii Hieron. & Polyphlebium & - \\
\hline gourlianum Grev. ex J. Sm. & Didymoglossum & Didymoglossum \\
\hline gracile Bosch & Crepidomanes & Crepidomanes/Gonocormus \\
\hline gracillimum Copel. & Crepidomanes & Crepidomanes/Crepidium \\
\hline grande Copel. & Crepidomanes & Nesopteris \\
\hline graucescens Bosch & Hymenophyllum & Pleuromanes \\
\hline guianense Sturm & Trichomanes & Lacostea \\
\hline guidoi P.G. Windisch & Trichomanes & Trichomanes \\
\hline guineense Afzel. ex Sw. & Abrodictyum & Pachychaetum \\
\hline haenkei C. Presl & Trichomanes & Trichomanes \\
\hline hartii Baker & Abrodictyum? & $?$ \\
\hline harveyi Carruth. ex Seem. & Crepidomanes & Nesopteris \\
\hline haughtii C.V. Morton & Polyphlebium & - \\
\hline henzaianum Parish ex Hook. & Didymoglossum & Didymoglossum \\
\hline l’herminieri Fée & Trichomanes & Trichomanes \\
\hline herzogii Rosenst. & Polyphlebium & - \\
\hline hibernicum A. Spreng. & Vandenboschia & Vandenboschia \\
\hline hieronymi Brause & Hymenophyllum & Hymenophyllum \\
\hline hildebrandtii Kuhn & Didymoglossum & Didymoglossum \\
\hline hirsutum $\mathrm{L}$. & Hymenophyllum & Sphaerocionium \\
\hline hispidulum Mett. ex Kuhn & Callistopteris & - \\
\hline hispidum Poir. & Hymenophyllum & Sphaerocionium \\
\hline holopterum Kunze & Trichomanes & Trichomanes \\
\hline hookeri C. Presl & Didymoglossum & Microgonium \\
\hline hosei Baker & Hymenophyllum & Hymenophyllum \\
\hline huberi H. Christ & Trichomanes & Trichomanes \\
\hline humile G. Forst. & Crepidomanes & Crepidomanes/Crepidium \\
\hline hygrometricum Poir. & Hymenophyllum & Sphaerocionium \\
\hline hymenoides Hedw. & Didymoglossum & Didymoglossum \\
\hline hymenophylloides Bosch & Polyphlebium & - \\
\hline hypnoides H. Christ & Polyphlebium & - \\
\hline idoneum C.V. Morton & Abrodictyum & Abrodictyum \\
\hline ignobile Ces. & Callistopteris? & $?$ \\
\hline imbricatum Sodiro & Trichomanes & Trichomanes \\
\hline inaequale Poir. & Hymenophyllum & Mecodium \\
\hline incisum Thunb. & Cyathea & - \\
\hline inerme Bosch & Hymenophyllum & Sphaerocionium \\
\hline
\end{tabular}


Appendix. Continued.

\begin{tabular}{|c|c|c|}
\hline Basionym & Genus & Subgenus / Section \\
\hline \multicolumn{3}{|l|}{ Trichomanes (cont.) } \\
\hline infundibulare Alderw. & Cephalomanes & - \\
\hline ingae C. Chr. & Polyphlebium & - \\
\hline intermedium Bosch & Crepidomanes & Nesopteris \\
\hline intramarginale Hook. \& Grev. & Crepidomanes & Crepidomanes/Crepidomanes \\
\hline intricatum Farrar & Vandenboschia? & ? \\
\hline japonicum Thunb. ex Murray & Onychium & - \\
\hline javanicum Blume & Cephalomanes & - \\
\hline jenmanii Lellinger & Trichomanes & Trichomanes \\
\hline johnstonense F.M. Bailey & Vandenboschia & Vandenboschia \\
\hline junceum H. Christ & Polyphlebium & - \\
\hline jungermannioides E. Fourn. & Polyphlebium & - \\
\hline kalamocarpum Hayata & Vandenboschia & Vandenboschia \\
\hline kalbreyeri Baker & Trichomanes & Trichomanes \\
\hline kapplerianum Sturm & Didymoglossum & Microgonium \\
\hline kaulfussii Hook. \& Grev. & Trichomanes & Trichomanes \\
\hline killipii Weath. & Trichomanes & Trichomanes \\
\hline kingii Copel. & Cephalomanes & - \\
\hline kirkii Hook. & Didymoglossum & Microgonium \\
\hline krausii Hook. \& Grev. & Didymoglossum & Didymoglossum \\
\hline krugii $\mathrm{H}$. Christ & Abrodictyum & Pachychaetum \\
\hline kunzeanum Hook. & Vandenboschia & Vandenboschia \\
\hline kurzii Bedd. & Crepidomanes & Crepidomanes/Crepidomanes \\
\hline labiatum Jenman & Didymoglossum & Didymoglossum \\
\hline laceratum Desv. & Trichomanes & Lacostea \\
\hline laciniatum Roxb. & Cephalomanes & - \\
\hline laciniosum Alston & Trichomanes & Trichomanes \\
\hline laetevirens Fée & Vandenboschia & Vandenboschia \\
\hline laetum Bosch & Abrodictyum & Pachychaetum \\
\hline lambertianum Hook. & Trichomanes & Trichomanes \\
\hline lanceolatum Poir. & Davallia & - \\
\hline lanceum Bory ex Willd. & Hymenophyllum & Sphaerocionium \\
\hline langsdorffii Bosch & Trichomanes & Trichomanes \\
\hline lasiophyllum Alderw. & Hymenophyllum & Hymenophyllum \\
\hline lastreoides C. Presl & Trichomanes & Davalliopsis \\
\hline latemarginale D.C. Eaton & Crepidomanes & Crepidomanes/Crepidomanes \\
\hline latevirens Fée & Vandenboschia & Vandenboschia \\
\hline latifrons Bosch & Crepidomanes & Crepidomanes/Crepidomanes \\
\hline latilabiatum E.D.Br. & Crepidomanes & Crepidomanes/Gonocormus \\
\hline latilobium Bonap. & Crepidomanes & Crepidomanes/Crepidomanes \\
\hline latipinnulata Bonap. & Trichomanes & $?$ \\
\hline latipinnum Copel. & Abrodictyum & Pachychaetum \\
\hline latisectum $\mathrm{H}$. Christ & Abrodictyum? & $?$ \\
\hline lauterbachii $\mathrm{H}$. Christ & Crepidomanes & Crepidomanes/Crepidium \\
\hline laxum Klotzsch & Trichomanes & Trichomanes \\
\hline lechleri Bosch & Polyphlebium & - \\
\hline ledermanni Brause & Cephalomanes & - \\
\hline lehmannii Hieron. & Didymoglossum & Didymoglossum \\
\hline lenormandi Bosch & Didymoglossum & Microgonium \\
\hline lentum Poir. & Davallia & - \\
\hline lepervanchii Cordem. & Crepidomanes? & Crepidomanes/Gonocormus? \\
\hline leprieurii Hook. & Trichomanes & Davalliopsis \\
\hline leptophyllum A. Cunn. & Abrodictyum & Abrodictyum \\
\hline
\end{tabular}


Appendix. Continued.

\begin{tabular}{|c|c|c|}
\hline Basionym & Genus & Subgenus / Section \\
\hline \multicolumn{3}{|l|}{ Trichomanes (cont.) } \\
\hline levissisum Fée & Vandenboschia? & $?$ \\
\hline liberiense Copel. & Didymoglossum & Didymoglossum \\
\hline lindenii C. Presl & Trichomanes & Trichomanes \\
\hline lindigii E. Fourn. & Trichomanes & Trichomanes \\
\hline lineare Sw. & Hymenophyllum & Sphaerocionium \\
\hline liukiuense Y. Yabe & Vandenboschia & Vandenboschia \\
\hline longicollum Bosch & Abrodictyum & Pachychaetum \\
\hline longifolium Desv. & Trichomanes & Trichomanes \\
\hline longifrons Nakai & Crepidomanes & Crepidomanes/Crepidomanes \\
\hline longilabiatum Bonap. & Crepidomanes & Crepidomanes/Crepidomanes? \\
\hline longisetum Bory ex Willd. & Abrodictyum & Pachychaetum \\
\hline lorencei Tardieu & Didymoglossum & Didymoglossum \\
\hline loreum Bory \& Bél. & Hymenophyllum & Sphaerocionium \\
\hline lozanii M.T. Murillo & Trichomanes & Trichomanes \\
\hline lucens $\mathrm{Sw}$. & Trichomanes & Trichomanes \\
\hline lucidum Roxb. & Davallia & - \\
\hline ludovicinum Rosenst. & Trichomanes & Trichomanes \\
\hline luerssenii F. Muell. & Abrodictyum & Pachychaetum \\
\hline luschnatianum C. Presl & Vandenboschia & Lacosteopsis \\
\hline luzonicum C. Presl & Crepidomanes & Crepidomanes/Crepidium \\
\hline macgillivrayi Baker & Hymenophyllum & Hymenophyllum \\
\hline macilentum Bosch & Trichomanes & Trichomanes \\
\hline macroclados Kunze & Trichomanes & Trichomanes \\
\hline majorae Watts & Crepidomanes & Crepidomanes/Crepidomanes \\
\hline makinoi C. Chr. & Crepidomanes & Crepidomanes/Crepidomanes \\
\hline malayanum Roxb. & Sphenomeris? & - \\
\hline malingii Hook.f. & Hymenophyllum & Sphaerocionium \\
\hline maluense Brause & Cephalomanes & - \\
\hline mandiocanum Raddi & Abrodictyum & Pachychaetum \\
\hline mannianum Mett. ex Kuhn & Crepidomanes & Crepidomanes/Gonocormus \\
\hline mannii Hook. & Crepidomanes & Crepidomanes/Gonocormus \\
\hline marchantioides Zippel ex Moritz & Didymoglossum & Didymoglossum \\
\hline marginatum Mett. & Abrodictyum & Pachychaetum \\
\hline martinezii Rovirosa & Vandenboschia & Vandenboschia \\
\hline martiusii C. Presl & Trichomanes & Trichomanes \\
\hline matthewii $\mathrm{H}$. Christ & Crepidomanes & Crepidomanes/Gonocormus \\
\hline maximum Blume & Vandenboschia & Vandenboschia \\
\hline megistostomum Copel. & Crepidomanes & Crepidomanes/Crepidomanes \\
\hline meifolium Bory ex Willd. & Abrodictyum & Pachychaetum \\
\hline melanopus Baker & Didymoglossum & Didymoglossum \\
\hline melanorhizon Hook. & Crepidomanes & Crepidomanes/Crepidomanes \\
\hline melanotrichum Schltdl. & Crepidomanes & Crepidomanes/Crepidomanes \\
\hline membranaceum $\mathrm{L}$. & Didymoglossum & Didymoglossum \\
\hline merrillii Copel. & Abrodictyum & Pachychaetum \\
\hline mettenii C. Chr. & Crepidomanes & Crepidomanes/Crepidomanes \\
\hline mexicanum Bosch & Vandenboschia & Vandenboschia \\
\hline micayense Hieron. & Trichomanes & Trichomanes \\
\hline microchilum Baker & Hymenophyllum & Hymenophyllum \\
\hline microlirion Copel. & Crepidomanes & Crepidomanes/Crepidomanes \\
\hline microphyllum Mett. ex Kuhn & Crepidomanes & Crepidomanes/Crepidomanes \\
\hline micropubescens Proctor & Didymoglossum & Didymoglossum \\
\hline mildbraedii Brause & Hymenophyllum & Hymenophyllum? \\
\hline
\end{tabular}


Appendix. Continued.

\begin{tabular}{|c|c|c|}
\hline Basionym & Genus & Subgenus / Section \\
\hline \multicolumn{3}{|l|}{ Trichomanes (cont.) } \\
\hline millefolium Desv. & Trichomanes & Davalliopsis \\
\hline milnei Bosch & Abrodictyum & Abrodictyum \\
\hline mindorense $\mathrm{H}$. Christ & Didymoglossum & Microgonium \\
\hline minimum Alderw. & Crepidomanes & Crepidomanes/Crepidomanes \\
\hline minutissimum Alderw. & Didymoglossum & Didymoglossum \\
\hline minutulum Gaudich. & Crepidomanes & Crepidomanes/Crepidomanes? \\
\hline minutum Blume & Crepidomanes & Crepidomanes/Gonocormus \\
\hline miyakei Y. Yabe & Vandenboschia & Vandenboschia \\
\hline molle E. Fourn. & Trichomanes & Trichomanes \\
\hline montanum Hook. & Didymoglossum & Didymoglossum \\
\hline moritzii Bosch & Vandenboschia & Lacosteopsis \\
\hline mosenii Lindm. & Didymoglossum & Didymoglossum \\
\hline motleyi Bosch & Didymoglossum & Didymoglossum \\
\hline mougeotii Bosch & Trichomanes & Feea \\
\hline multifidum G. Forst. & Hymenophyllum & Hymenophyllum \\
\hline muscoides Sw. & Didymoglossum & Didymoglossum \\
\hline musolense Brause & Crepidomanes & Crepidomanes/Gonocormus? \\
\hline myrioneuron Lindm. & Didymoglossum & Didymoglossum \\
\hline myriophyllum Desv. & $?$ & $?$ \\
\hline myrioplasium Kunze & Callistopteris & - \\
\hline naseanum $\mathrm{H}$. Christ & Vandenboschia & Vandenboschia \\
\hline naumannii Kuhn \& Luerss. ex H. Christ & Polyphlebium & - \\
\hline neesii Blume & Hymenophyllum & Hymenophyllum \\
\hline neilgherrense Bedd. & Didymoglossum & Microgonium \\
\hline nipponicum Nakai & Vandenboschia & Vandenboschia \\
\hline nitidulum Bosch & Hymenophyllum & Sphaerocionium \\
\hline niveum Burm.f. & $?$ & $?$ \\
\hline novoguineense Brause & Crepidomanes & Crepidomanes/Gonocormus \\
\hline nudum Poir. & Hymenophyllum & Sphaerocionium \\
\hline nymanii $\mathrm{H}$. Christ & Crepidomanes & Crepidomanes/Crepidomanes \\
\hline obscurum Blume & Abrodictyum & Pachychaetum \\
\hline olivaceum Kunze ex Klotzsch & Polyphlebium & - \\
\hline opacum Bosch & Trichomanes & Davalliopsis \\
\hline orbiculare $\mathrm{H}$. Christ & Didymoglossum & Didymoglossum \\
\hline orientale C. Chr. & Vandenboschia & Vandenboschia \\
\hline ornatulum Bosch & Didymoglossum? & $?$ \\
\hline osmundoides Poir. & Trichomanes & Feea \\
\hline pabstianum Müll.Berol. & Didymoglossum & Didymoglossum \\
\hline pachyphlebium C. Chr. & Trichomanes? & Davalliopsis? \\
\hline pacificum Hedw. & Hymenophyllum & Hymenophyllum \\
\hline padronii Proctor & Trichomanes & Trichomanes \\
\hline pallidum Blume & Hymenophyllum & Pleuromanes \\
\hline palmatifidum Müll.Berol. & Hymenophyllum & Sphaerocionium \\
\hline palmatum C. Presl & Crepidomanes & Crepidomanes/Gonocormus \\
\hline palmicola Bosch & Didymoglossum & Microgonium \\
\hline palmifolium Hayata & Crepidomanes & Crepidomanes/Crepidomanes \\
\hline paniculatum Alderw. & Crepidomanes & Crepidomanes/Crepidomanes \\
\hline pannosum Ces. & Didymoglossum & Didymoglossum \\
\hline papillatum Müll.Berol. & Abrodictyum & Pachychaetum \\
\hline pappei Kunze & Abrodictyum & Pachychaetum \\
\hline papuanum Brause & Didymoglossum & Didymoglossum \\
\hline paradoxum Domin & Didymoglossum & Didymoglossum \\
\hline
\end{tabular}


Appendix. Continued.

\begin{tabular}{|c|c|c|}
\hline Basionym & Genus & Subgenus / Section \\
\hline \multicolumn{3}{|l|}{ Trichomanes (cont.) } \\
\hline parviflorum Poir. & Abrodictyum & Pachychaetum \\
\hline parvulum Poir. & Hymenophyllum & Hymenophyllum \\
\hline parvum Copel. & Vandenboschia & Vandenboschia \\
\hline pedicellatum Desv. & Trichomanes & Lacostea \\
\hline pellucens Kunze & Trichomanes & Trichomanes \\
\hline pellucidum Goldm. & Vandenboschia & Lacosteopsis \\
\hline peltatum Poir. & Hymenophyllum & Hymenophyllum \\
\hline pennatum Kaulf. & Trichomanes & Trichomanes \\
\hline perpusillum Alderw. & Crepidomanes & Crepidomanes/Crepidium? \\
\hline pervenulosum Alderw. & Crepidomanes & Crepidomanes/Crepidomanes \\
\hline petersii A. Gray & Didymoglossum & Didymoglossum \\
\hline philippianum Sturm & Polyphlebium & - \\
\hline piliferum Alderw. & Hymenophyllum & Sphaerocionium \\
\hline pilosum Raddi & Trichomanes & Trichomanes \\
\hline pinnatifidum Bosch & Trichomanes & Trichomanes \\
\hline pinnatinervium Jenman & Didymoglossum & Didymoglossum \\
\hline pinnatum Hedw. & Trichomanes & Trichomanes \\
\hline platyderon E. Fourn. & Abrodictyum & Pachychaetum \\
\hline platyrachis Domin & Trichomanes & Feea \\
\hline pluma Hook. & Abrodictyum & Abrodictyum \\
\hline plumosum Kunze & Trichomanes & Trichomanes \\
\hline plumula C. Presl & Trichomanes & Trichomanes \\
\hline poeppigii C. Presl & Trichomanes & Trichomanes \\
\hline polyanthos Sw. & Hymenophyllum & Mecodium \\
\hline polyodon Colenso & Abrodictyum & Pachychaetum \\
\hline polyphlebius V. Marcano & Trichomanes & Trichomanes \\
\hline polypodioides $\mathrm{L}$. & Trichomanes & Trichomanes \\
\hline polysperma Poir. & Sphenomeris & - \\
\hline polystromaticum Bierh. & Abrodictyum & Pachychaetum \\
\hline powellii Baker & Crepidomanes & Crepidomanes/Crepidomanes \\
\hline preslianum Nakai & Crepidomanes & Nesopteris \\
\hline preslii C.V. Morton & Cephalomanes & - \\
\hline prieurii Kunze & Trichomanes & Davalliopsis \\
\hline procerum Fée & Hymenophyllum & Sphaerocionium \\
\hline proliferum Blume & Crepidomanes & Crepidomanes/Gonocormus \\
\hline pseudo-arbuscula Alderw. & Abrodictyum & Pachychaetum \\
\hline pseudoblepharistomum Tagawa & Crepidomanes & Nesopteris \\
\hline pseudocapillatum Alderw. & Crepidomanes & Crepidomanes/Crepidium? \\
\hline ptilodes Bosch & Trichomanes & Trichomanes \\
\hline pulchellum Salisb. & Hymenophyllum & Hymenophyllum \\
\hline pulcherrimum Copel. & Crepidomanes & Nesopteris \\
\hline pumilum Bosch & Abrodictyum & Pachychaetum \\
\hline punctatum Poir. & Didymoglossum & Didymoglossum \\
\hline pusillum $\mathrm{Sw}$ & Didymoglossum & Didymoglossum \\
\hline pygmaeum C. Chr. & Didymoglossum & Microgonium \\
\hline pyxidiferum $\mathrm{L}$. & Polyphlebium & - \\
\hline quelpaertense Nakai & Vandenboschia & Vandenboschia \\
\hline quercifolium Desv. & Trichomanes & Trichomanes \\
\hline racemulosum Bosch & Abrodictyum & Pachychaetum \\
\hline radicans $\mathrm{Sw}$. & Vandenboschia & Vandenboschia \\
\hline ramitrichum Faden & Crepidomanes & Crepidomanes/Crepidomanes \\
\hline recedens Rosenst. & Crepidomanes & Crepidomanes/Crepidomanes \\
\hline
\end{tabular}


Appendix. Continued.

\begin{tabular}{|c|c|c|}
\hline Basionym & Genus & Subgenus / Section \\
\hline \multicolumn{3}{|l|}{ Trichomanes (cont.) } \\
\hline reniforme G. Forst. & Hymenophyllum & Cardiomanes \\
\hline repens Schott ex Sturm & Vandenboschia & Vandenboschia \\
\hline reptans $\mathrm{Sw}$. & Didymoglossum & Didymoglossum \\
\hline resinosum R.C. Moran & Trichomanes? & Davalliopsis? \\
\hline rhipidophyllum Sloss. & Didymoglossum & Didymoglossum \\
\hline rhizophyllum Cav. & Trichomanes & Trichomanes \\
\hline ridleyi Copel. & Hymenophyllum & Sphaerocionium \\
\hline rigidum $\mathrm{Sw}$ & Abrodictyum & Pachychaetum \\
\hline robinsonii Hook. ex Baker & Didymoglossum & Didymoglossum \\
\hline robustum E. Fourn. & Trichomanes & Trichomanes \\
\hline roemerianum Rosenst. & Crepidomanes & Nesopteris \\
\hline roraimense Jenman & Trichomanes & Trichomanes \\
\hline rosenstockii Alderw. & Cephalomanes & - \\
\hline rothertii Alderw. & Crepidomanes & Crepidomanes/Crepidomanes \\
\hline rotundifolium Bonap. & Didymoglossum & Microgonium \\
\hline rupicolum Racib. & Crepidomanes & Crepidomanes/Crepidomanes \\
\hline ruwenzoriense Taton & Crepidomanes & Crepidomanes/Gonocormus \\
\hline samoense C. Chr. & Crepidomanes & Crepidomanes/Crepidomanes \\
\hline sandvicense Bosch & Vandenboschia & Vandenboschia \\
\hline sanguinolentum $\mathrm{G}$. Forst. & Hymenophyllum & Myrmecostylum \\
\hline savaiense Lauterb. & Hymenophyllum & Pleuromanes \\
\hline saxatile Brack. ex T. Moore & Abrodictyum & Pachychaetum \\
\hline saxifragoides $\mathrm{C}$. Presl & Crepidomanes & Crepidomanes/Gonocormus \\
\hline sayeri F. Muell. \& Baker & Didymoglossum & Didymoglossum \\
\hline scandens $\mathrm{L}$. & Trichomanes & Trichomanes \\
\hline schaffneri Schltdl. & Didymoglossum & Didymoglossum \\
\hline schiedeanum Müll.Berol. & Polyphlebium & - \\
\hline schlechteri Brause & Abrodictyum & Abrodictyum \\
\hline schmidianum Zenker ex Taschner & Crepidomanes & Crepidomanes/Crepidomanes \\
\hline schomburgkianum Sturm & Trichomanes & Trichomanes \\
\hline schomburgkii Bosch & Trichomanes & Trichomanes \\
\hline schultzei Brause & Abrodictyum & Abrodictyum \\
\hline seemannii Carruth. ex Seem. & Abrodictyum & Pachychaetum \\
\hline sellowianum C. Presl & Trichomanes & Trichomanes \\
\hline sericeum $\mathrm{Sw}$. & Hymenophyllum & Sphaerocionium \\
\hline serratifolium Rosenst. & Polyphlebium & - \\
\hline serratulum Baker & Hymenophyllum & Hymenophyllum \\
\hline serricula Fée & Trichomanes & Trichomanes \\
\hline setaceum Bosch & Abrodictyum & Pachychaetum \\
\hline setiferum Baker ex Jenman & Didymoglossum & Didymoglossum \\
\hline setigerum Backh. ex T. Moore & Abrodictyum & Pachychaetum \\
\hline siamense $\mathrm{H}$. Christ & Abrodictyum & Pachychaetum \\
\hline sibthorpioides Bory ex Willd. & Hymenophyllum & Hymenophyllum \\
\hline sinuatum Bonap. & Didymoglossum & Microgonium \\
\hline sinuosum Rich. ex Willd. & Trichomanes & Trichomanes \\
\hline smithii Hook. & Abrodictyum & Abrodictyum \\
\hline societense J.W. Moore & Callistopteris & - \\
\hline solidum $\mathrm{G}$. Forst. & Davallia & - \\
\hline solitarium Jenman & Didymoglossum & Didymoglossum \\
\hline somai Nakai & Vandenboschia & Vandenboschia \\
\hline sonderi Bosch & Trichomanes & Lacostea \\
\hline speciosum Willd. & Vandenboschia & Vandenboschia \\
\hline
\end{tabular}


Appendix. Continued.

\begin{tabular}{|c|c|c|}
\hline Basionym & Genus & Subgenus / Section \\
\hline \multicolumn{3}{|l|}{ Trichomanes (cont.) } \\
\hline spectabile Klotzsch & Trichomanes & Trichomanes \\
\hline sphenoides Kunze & Didymoglossum & Didymoglossum \\
\hline spicatum Hedw. ex Hook. & Trichomanes & Feea \\
\hline spicisorum Desv. & Trichomanes & Feea \\
\hline spinulosum Phil. & Hymenophyllum & Hymenophyllum \\
\hline splendidum Bosch & Trichomanes & Trichomanes \\
\hline spruceanum Hook. & Trichomanes & Trichomanes \\
\hline sprucei Baker & Abrodictyum & Pachychaetum \\
\hline squarrosum G. Forst. & Dicksonia & - \\
\hline stenosiphon $\mathrm{H}$. Christ & Vandenboschia & Vandenboschia \\
\hline steyermarkii P.G. Windisch \& A.R. Sm. & Trichomanes & Trichomanes \\
\hline striatum D. Don & Vandenboschia? & $?$ \\
\hline strictum Menzies ex Hook. \& Grev. & Abrodictyum & Abrodictyum \\
\hline strigosum Thunb. ex Murray & Microlepia & - \\
\hline stylosum Poir. & Abrodictyum & Pachychaetum \\
\hline subdeltoideum $\mathrm{C}$. Chr. & Abrodictyum & Pachychaetum \\
\hline subexsertum Bosch & Polyphlebium & - \\
\hline sublabiatum Bosch & Trichomanes & Trichomanes \\
\hline sublimbatum Müll.Berol. & Didymoglossum & Didymoglossum \\
\hline subpinnatifidum Bosch & Crepidomanes & Crepidomanes/Gonocormus \\
\hline subsessile Splitg. & Trichomanes & Lacostea \\
\hline subtilissimum Brause & Crepidomanes & Crepidomanes/Gonocormus \\
\hline subtrifidum C.G. Matthew \& H. Christ & Crepidomanes & Crepidomanes/Gonocormus \\
\hline suffrutex Alderw. & Cephalomanes & - \\
\hline sumatranum Alderw. & Cephalomanes & - \\
\hline superbum Backh. ex T. Moore & Callistopteris & - \\
\hline taeniatum Copel. & Hymenophyllum & Sphaerocionium \\
\hline tahitense Nadeaud & Didymoglossum & Didymoglossum \\
\hline tamarisciforme Jacq. & Abrodictyum & Pachychaetum \\
\hline tanaicum Hook. & Trichomanes & Lacostea \\
\hline tenellum Hedw. & Polyphlebium & - \\
\hline tenerum A. Spreng. & Polyphlebium & - \\
\hline tenue Brack. & Polyphlebium & - \\
\hline tenuifolium Burm.f. & Cheilanthes & - \\
\hline tenuissimum Bosch & Polyphlebium & - \\
\hline tereticaulum Ching & Abrodictyum & Pachychaetum \\
\hline teysmanni Bosch & Crepidomanes & Crepidomanes/Gonocormus \\
\hline thouarsianum C. Presl & Hymenophyllum & Hymenophyllum \\
\hline thuioides Desv. & Abrodictyum & Pachychaetum \\
\hline thysanostomum Makino & Crepidomanes & Nesopteris \\
\hline tomaniiviense Brownlie & Hymenophyllum & Sphaerocionium \\
\hline torotumani Vareschi & Trichomanes & Lacostea \\
\hline tosae H. Christ ex Matsum. & Crepidomanes & Crepidomanes/Crepidomanes \\
\hline tranninense Fée & Polyphlebium & - \\
\hline translucens Kunze & Polyphlebium & - \\
\hline trichodes Sw. & Polyphlebium & - \\
\hline trichoideum $\mathrm{Sw}$ & Polyphlebium & - \\
\hline trichophorum Alderw. & Hymenophyllum & Hymenophyllum \\
\hline trichophyllum T. Moore & Abrodictyum & Pachychaetum \\
\hline trigonum Desv. & Trichomanes & Trichomanes \\
\hline trinerve Baker & Crepidomanes & Crepidomanes/Gonocormus \\
\hline trollii Bergdolt & Trichomanes & Feea \\
\hline
\end{tabular}


Appendix. Continued.

\begin{tabular}{|c|c|c|}
\hline Basionym & Genus & Subgenus / Section \\
\hline \multicolumn{3}{|l|}{ Trichomanes (cont.) } \\
\hline tuerckheimii H. Christ & Trichomanes & Lacostea \\
\hline tunbrigense L. & Hymenophyllum & Hymenophyllum \\
\hline ujhelyii Kümmerle & Trichomanes & Trichomanes \\
\hline ulei $\mathrm{H}$. Christ & Polyphlebium? & - \\
\hline undulatum Sw. & Hymenophyllum & Mecodium \\
\hline uniflorum Cav. & Crepidomanes? & Nesopteris? \\
\hline vandenboschii P.G. Windisch & Trichomanes & Trichomanes \\
\hline varians Alderw. & Didymoglossum & Microgonium \\
\hline vaupelii Brause & Crepidomanes? & Crepidomanes/Crepidomanes? \\
\hline vaupesensis Lellinger & Trichomanes & Trichomanes \\
\hline venosum $\mathrm{R} . \mathrm{Br}$. & Polyphlebium & - \\
\hline venustulum Colenso & Polyphlebium & - \\
\hline venustum Desv. & Vandenboschia & Lacosteopsis \\
\hline vestitum Baker & Hymenophyllum & Hymenophyllum \\
\hline vieillardii Bosch & Polyphlebium & - \\
\hline virgatulum Bosch & Vandenboschia & Vandenboschia? \\
\hline viridans Mett. ex Kuhn & Crepidomanes & Crepidomanes/Crepidomanes \\
\hline viride Bubani & Asplenium & - \\
\hline vitiense Baker & Crepidomanes & Crepidomanes/Crepidomanes \\
\hline vittaria DC. ex Poir. & Trichomanes & Trichomanes \\
\hline volubile Vell. & Trichomanes & Lacostea \\
\hline walleri Watts & Crepidomanes & Crepidomanes/Crepidomanes \\
\hline wallii Thwaites ex Trimen & Didymoglossum & Didymoglossum \\
\hline warburgii $\mathrm{H}$. Christ & Abrodictyum & Abrodictyum \\
\hline weddellii Bosch & Trichomanes & Davalliopsis \\
\hline werneri Rosenst. & Polyphlebium & - \\
\hline wildii F.M. Bailey & Crepidomanes & Crepidomanes/Crepidium \\
\hline windischianum Lellinger & Abrodictyum & Pachychaetum \\
\hline yandinense F.M. Bailey & Didymoglossum & Microgonium \\
\hline zollingeri Bosch & Cephalomanes & - \\
\hline \multicolumn{3}{|l|}{ Vandenboschia } \\
\hline assimilis Ching \& P.C. Chiu & Vandenboschia & Vandenboschia \\
\hline inopinata Pic.Serm. & Crepidomanes & Crepidomanes/Crepidomanes \\
\hline lofoushanensis Ching & Vandenboschia & Vandenboschia? \\
\hline subclathrata $\mathrm{K}$. Iwats. & Vandenboschia & Vandenboschia \\
\hline titibuensis H. Itô & Crepidomanes & Crepidomanes/Crepidomanes \\
\hline tubiflora F.S. Wagner & Vandenboschia & Vandenboschia \\
\hline
\end{tabular}

